\title{
USING THE SINGULARITY TRACE TO UNDERSTAND LINKAGE MOTION CHARACTERISTICS
}

\author{
Thesis \\ Submitted to \\ The School of Engineering of the \\ UNIVERSITY OF DAYTON \\ In Partial Fulfillment of the Requirements for \\ The Degree of \\ Master of Science in Mechanical Engineering \\ By \\ Lin $\mathrm{Li}$ \\ UNIVERSITY OF DAYTON \\ Dayton, Ohio
}

May, 2013 


\title{
USING THE SINGULARITY TRACE TO UNDERSTAND \\ LINKAGE MOTION CHARACTERISTICS
}

Name: Li, Lin

APPROVED BY:

Andrew Murray, Ph.D.

David Myszka, Ph.D.

Advisor Committee Chairman

Committee Member

Professor, Mechanical and Aerospace

Associate Professor, Mechanical and

Engineering Department

Aerospace Engineering Department

\begin{abstract}
Ahmad Kashani, Ph.D.
Committee Member

Professor, Mechanical and Aerospace

Engineering Department
\end{abstract}

John Weber, Ph.D.

Associate Dean

School of Engineering
Tony E. Saliba, Ph.D.

Dean, School of Engineering

\& Wilke Distinguished Professor 


\section{(C) Copyright by}

Lin Li

All rights reserved

2013 


\section{ABSTRACT \\ USING THE SINGULARITY TRACE TO UNDERSTAND \\ LINKAGE MOTION CHARACTERISTICS}

Name: Li, Lin

University of Dayton

Advisor: Dr. Andrew Murray

This thesis provides examples of a new method used to analyze the motion characteristics of single-degree-of-freedom, closed-loop linkages with a designated input angle and one or two design parameters. The method involves the construction of a singularity trace, which is a plot that reveals changes in the number of geometric inversions, singularities, and changes in the number of branches as a design parameter is varied. This thesis applies the method to planar linkages such as the Watt II, Stephenson III and double butterfly, and spatial linkages such as spherical four-bar and RevoluteCylindrical-Cylindrical-Cylindrical (RCCC) linkages.

Results from this investigation include the following. Special instances of the singularity trace for the Watt II linkage include multiple coincident projections of the singularity curve and symmetric characteristics of the singularity trace for special design parameters. For the Stephenson III and double butterfly linkages, instances where the input angle is able to rotate more than one revolution between singularities have been identified. This characteristic demonstrates a net-zero, singularity free activation sequence that places the mechanism into a different geometric inversion. Additional observations from the examples show that net-zero, singularity free activation sequences can occur 
in the motion curves with "U" or " $\mathrm{S}$ " shapes. Cases are shown where subtle changes to two design parameters of a Stephenson III linkage drastically alter the motion. Additionally, isolated critical points are found to exist for the double butterfly, where the linkage becomes a structure and looses the freedom to move.

This thesis also applies this method to spatial linkages to analyze a spherical mechanism. The structure of the loop closure equations was modified to accommodate a third, spatial coordinate. An example is provided that uses a spherical four-bar linkage. The singularity map has been found to be similar to a comparable planar four-bar case.

Lastly, loop closure and singularity equations have been formulated to address a truly spatial linkage, the RCCC. A solution method has been identified. The analysis is listed in future work. 
To my parents 


\section{ACKNOWLEDGMENTS}

My special thanks are in order to Dr. Murray and Dr. Myszka, my advisers, for providing the time and equipment necessary for the work contained herein, and for directing this thesis and bringing it to its conclusion with patience and expertise.

I would like to express my appreciation to everyone who has helped me with this work. This includes Jon Lauden, who offered guidance with many practical issues; Shamsul Anuar Bin Shamsudin, for patiently preparing and correcting the text; Yuxuan Tong, who gave advice concerning the calculation; Joshua Nieman, Ali Almandeel and Bingjue Li, who gave me courage and support during the research. 


\section{TABLE OF CONTENTS}

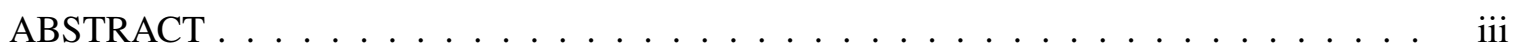

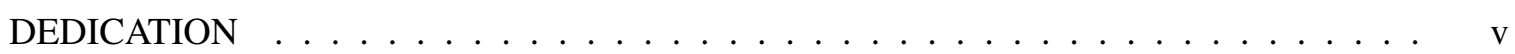

ACKNOWLEDGMENTS . . . . . . . . . . . . . . . . . . . . vi

LIST OF FIGURES $\ldots \ldots \ldots \ldots \ldots \ldots \ldots \ldots \ldots \ldots \ldots$ ix

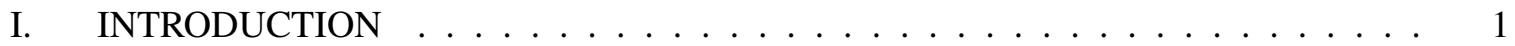

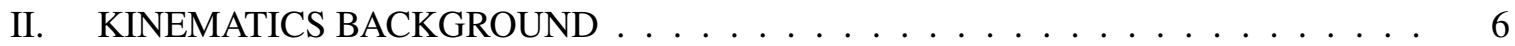

2.1 Loop Closure: Component Method . . . . . . . . . . . . . . . . . 6

2.2 Loop Closure: Exponent Method . . . . . . . . . . . . . . . . . . . . . . . 9

2.3 Isotropic Coordinates . . . . . . . . . . . . . . . . . . . . . . 10

2.4 Geometric Analysis of Singularities . . . . . . . . . . . . . . . . . . . 12

2.5 Transition Linkage . . . . . . . . . . . . . . . . . . . . . . . . . . . 14

2.6 Spatial Kinematics . . . . . . . . . . . . . . . . . . . . . . 15

2.6 .1 Rotations . . . . . . . . . . . . . . . . . . 15

2.6.2 Spatial Displacements . . . . . . . . . . . . . . 16

III. SINGULARITY POINTS, MOTION CURVE, CRITICAL POINTS, SINGULARITY TRACE AND DESIGN SURFACE . . . . . . . . . . . . . . . . . 19

3.1 Forward Kinematics . . . . . . . . . . . . . . . . . . 22

3.2 Find all Singularity Points . . . . . . . . . . . . . . . . 22

3.3 Find all Branches . . . . . . . . . . . . . . . . . . . 25

3.4 Find Critical Points . . . . . . . . . . . . . . . . . . . . 27

3.5 Generate the Singularity Trace . . . . . . . . . . . . . . . 30 
IV. ANALYSIS EXAMPLES OF TYPICAL LINKAGES . . . . . . . . . . . . . . . 32

4.1 Watt II Linkage . . . . . . . . . . . . . . . . . . . . . . . . . 32

4.1 .1 Loop Closure . . . . . . . . . . . . . . . . . . . . . . . . . . . . . . 33

4.1 .2 Forward Kinematics . . . . . . . . . . . . . . . . . . . . 34

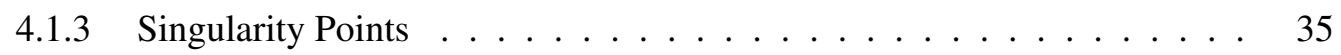

4.1 .4 Motion Curve . . . . . . . . . . . . . . . . . 35

4.1 .5 Critical Points . . . . . . . . . . . . . . 36

4.1 .6 Singularity Trace . . . . . . . . . . . . . . . . . . . . . . . . . . . . . . . . 38

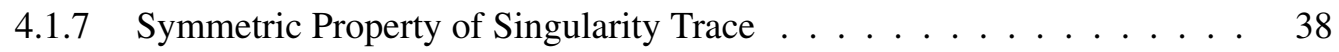

4.2 Stephenson III Linkage . . . . . . . . . . . . . . . . . . . . . . . . . 41

$4.2 .1 \quad$ Critical Points . . . . . . . . . . . . . . . . 43

4.2 .2 Singularity Trace . . . . . . . . . . . . . . . . . 43

4.2 .3 Motion Curves . . . . . . . . . . . . . . . . . . . 44

4.2 .4 Design Surface . . . . . . . . . . . . . . . . . . . . . . . 49

4.3 Double Butterfly Linkage . . . . . . . . . . . . . . . . . . . . 50

4.3 .1 Loop Closure . . . . . . . . . . . . . . . . . . . . . . . . . . . . . . . 50

4.3 .2 Forward Kinematics . . . . . . . . . . . . . . . . . . . 52

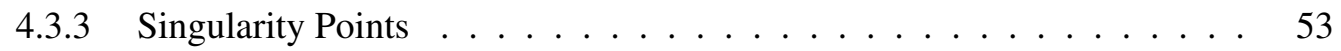

4.3 .4 Motion Curve . . . . . . . . . . . . . . . . 53

4.3 .5 Critical Points . . . . . . . . . . . . . . . . 55

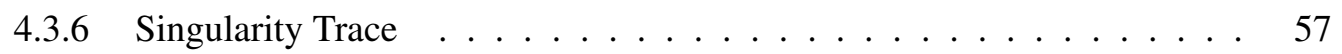

4.4 Spherical Four-Bar Linkage $\ldots \ldots \ldots \ldots$

4.4.1 Forward Kinematics . . . . . . . . . . . . . . . . . . 59

4.4 .2 Singularity Points . . . . . . . . . . . . . . . 62

4.4 .3 Motion Curve . . . . . . . . . . . . . . . . 63

4.4 .4 Critical Points . . . . . . . . . . . . . . . . . 64

4.4 .5 Singularity Trace . . . . . . . . . . . . . . . 65

V. CONCLUSION AND FUTURE WORK . . . . . . . . . . . . . . . . . . 67

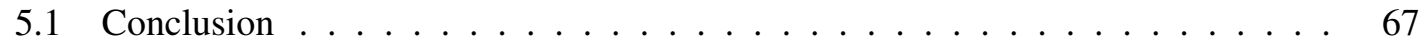

5.2 Future Work . . . . . . . . . . . . . . . . . . 67

$5.2 .1 \quad$ Forward Kinematics . . . . . . . . . . . . . . . . . 68

5.2 .2 Singularity Points . . . . . . . . . . . . . 70

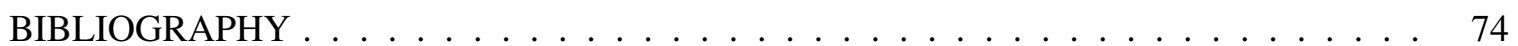




\section{LIST OF FIGURES}

$2.1 \quad$ A 4 R linkage. . . . . . . . . . . . . . . . . . . 7

2.2 A 4 R linkage with two different GIs. . . . . . . . . . . . . . . 8

2.3 A unit vector plotted on the complex plane. . . . . . . . . . . . . 9

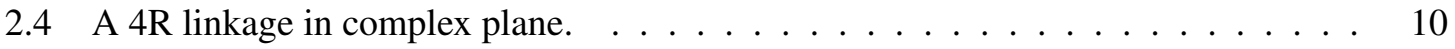

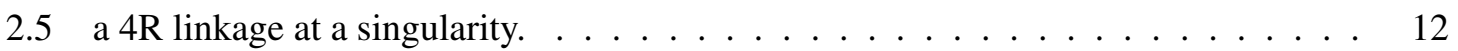

2.6 The same $4 \mathrm{R}$ linkage at another singularity . . . . . . . . . . . . . . . 13

2.7 A Stephenson III linkage at a singularity. . . . . . . . . . . . . . . . . . . . . 14

2.8 The fixed and moving frames defining spatial rotations about the $x$-axis and $z$-axis. $\quad 16$

2.9 Coordinate screw displacement along $x$-axis. $\ldots \ldots \ldots \ldots \ldots$

2.10 Coordinate screw displacement along $z$-axis. . . . . . . . . . . . . . 18

3.1 The motion curve for a four-bar mechanism as expressed in Eq. 3.5 or 3.6. . . . . . 21

3.2 Projections of the motion curve from Fig. 3.1. Turning points, shown with red markers, have no $x\left(\theta_{2}\right.$ in this case $)$ component. . . . . . . . . . .

3.3 Projections of the singularity trace of the four-bar linkage, critical points, shown with red markers, have no $x\left(a_{1}\right.$ in this case $)$ component. . . . . . . . . . . .

4.1 Watt II linkage position vector loop. . . . . . . . . . . . . . .

4.2 Trace of the motion curve for the Watt II linkage, with $a_{1}=2.0$, projected onto the

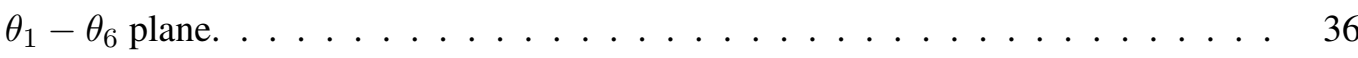


4.3 Trace of the motion curve for the Watt II linkage, with $a_{1}=9.0$, projected onto the

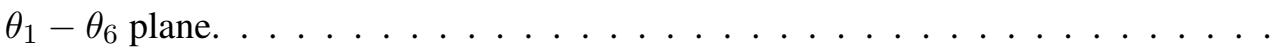

4.4 Projection of the Watt II singularity trace. Red circles mark the critical points. Regions of equal GIs and circuits are identified. . . . . . . . . . . . . . . .

4.5 Projection of the Watt II singularity trace. Red circles mark the critical points. Regions of equal GIs and circuits are identified. . . . . . . . . . . . . . . . . 40

4.6 Watt II linkage with $a_{1}=3.5$, motion curve trace on $\theta_{1}-\theta_{6}$ plane. . . . . . . . 41

4.7 Watt II linkage with $a_{1}=10.0$, motion curve trace on $\theta_{1}-\theta_{6}$ plane. . . . . . . . 42

4.8 Stephenson III linkage position vector loop. . . . . . . . . . . . . . . . . . . 44

4.9 Projection of the Stephenson III singularity trace with respect to $a_{1}$. Red circles mark the critical points. Regions of equal GIs and circuits are identified. . . . . . .

4.10 Stephenson III linkage motion curve with respect to $\theta_{1}, \theta_{3}$ at $a_{1}=1.1 \ldots \ldots$. . . . 46

4.11 Stephenson III linkage motion curve with respect to $\theta_{1}, \theta_{3}$ at $a_{1}=2.0 \ldots \ldots$. . . 46

4.12 Stephenson III singularity trace with respect to $a_{4} \ldots \ldots \ldots \ldots \ldots \ldots$

4.13 Stephenson III motion curve with respect to $a_{1}=2.09, a_{4}=4.15 \ldots \ldots \ldots \ldots$

4.14 Stephenson III design surface is created by stacking motion curves with respect to $\theta_{1}$ and $\theta_{3}$ for increasing values of $a_{1} \ldots \ldots \ldots \ldots$

4.15 Double butterfly linkage position vector loop. . . . . . . . . . . . . . 50

4.16 Trace of the motion curve for the double butterfly linkage, with $a_{1}=0.198$, projected onto the $\theta_{1}-\theta_{2}$ plane. . . . . . . . . . . . . . 54

4.17 Trace of the motion curve for the double butterfly linkage, with $a_{1}=0.40$, projected

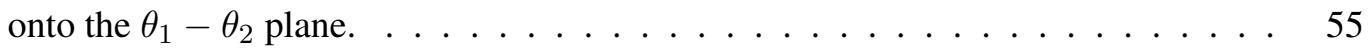

4.18 Trace of the motion curve for the double butterfly linkage, with $a_{1}=2.50$, projected

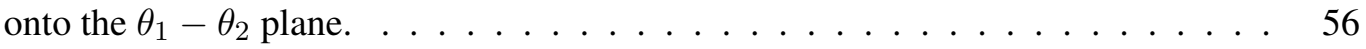

4.19 Trace of the motion curve for another double butterfly linkage with different param-

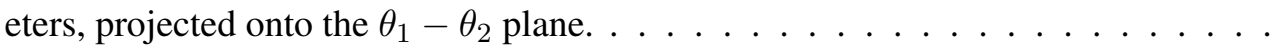


4.20 Double butterfly linkage singularity trace with respect to $a_{1} \ldots \ldots \ldots$

4.21 Double butterfly isolated critical position at P3 . . . . . . . . . . 59

4.22 Double butterfly linkage singularity trace with respect to $a_{3} \ldots \ldots \ldots$

4.23 Spherical Four-Bar linkage. . . . . . . . . . . . . . . . . . . 61

4.24 Spherical four-bar linkage singularity position. . . . . . . . . . . 63

4.25 Trace of the motion curve for the spherical four-bar linkage, with $\alpha=1.5$, projected onto the $\theta_{1}-\theta_{2}$ plane. . . . . . . . . . . . . . . . . 64

4.26 Projection of the spherical four-bar linkage singularity trace with respect to $\alpha$. Red circles mark the critical points. Regions of equal GIs and circuits are identified. . .

4.27 Projection of the spherical four-bar linkage singularity trace with respect to $\alpha$. Red circles mark the critical points. Regions of equal GIs and circuits are identified. . . 66

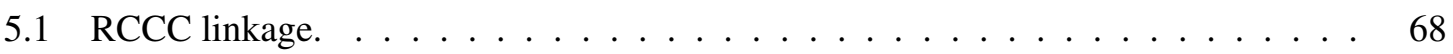

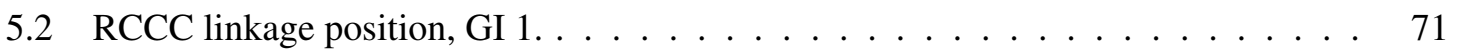

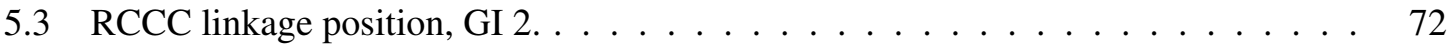




\section{CHAPTER I}

\section{INTRODUCTION}

The automation of repetitive tasks is a critical part of modern manufacturing and assembly processes. Robotics is one widely adopted solution, but comes with high associated maintenance and energy costs. Automation of these repetitive tasks may also be accomplished by dissecting them into a sequence of simple tasks solvable by lower complexity devices. Lower complexity devices typically exhibit high reliability and low cost, but are dedicated to solving one problem. These lower complexity devices, if designed to be reconfigurable, offer the potential advantages of both robotics and dedicated machinery. The broad goal of this research is to analyze a variety of reconfigurable linkages for use in flexible automation tasks and produce the principles for their design.

More precisely, this thesis implements motion analysis theory utilizing isotropic coordinates to allow for the construction of mathematical models of planar linkages composed of rigid bodies, revolute joints, and prismatic joints. A graphical representation has been developed to display the gross motion characteristics of linkages called a singularity trace [1]. The singularity trace provides a visual snapshot of the effects of the reconfigurability on the linkage by including the number of assembly circuits and the locations of the locked configurations. A tool adept at working with large algebraic systems of equations, Bertini [2], has been applied to allow for the solution to the complex systems arising in the design challenges of this analysis. MATLAB is then used to process 
the Bertini solutions and construct the complete singularity map. The detailed description of the motion characteristics explored throughout this thesis are provided below.

Direct (or forward) kinematic position analysis of a single degree-of-freedom (DOF) linkage involves determining values for joint parameters for a given position of the input link. Since the governing kinematic constraint equations for the links in a closed-loop linkage are non-linear, multiple solutions are obtained for a single position of the input link. Erdman et. al., [3] refer to each solution as a geometric inversion (GI) and each is associated with an alternate configuration of the mechanism at the specific input. The analysis method used in this thesis includes the planar analysis theory which presents techniques for using isotropic coordinates to generate polynomial equations that describe the position of a general single-DOF linkage. Solution of the complex, polynomial systems can be readily accomplished by using numerical polynomial continuation [4]. The set of position equations is represented by the motion curve, whose trace is created by plotting the GIs for various positions of the input link. The trace of the motion curve for planar linkages of more than four bars can be quite convoluted involving multiple segments.

A circuit of a mechanism is defined by Chase and Mirth [5] as the set of all possible orientations of links that can be realized without disconnecting any of the joints. If a mechanism must be disassembled to move from one position to another, the two positions reside on separate circuits. Each segment on the trace of the motion curve represents a different circuit. At a specific input link position, each GI may lie on a different circuit, but several GIs can lie on the same circuit. The circuits of a mechanism are independent of the choice of driving link, and there is no direct relationship between the geometric inversions and circuits [5]. Established methods to identify different circuits rely on geometric insights of a particular mechanism $[6,7,8,9,10,11]$.

Singularity points exist when the driving link is no longer able to move the mechanism. At the singularity points, also called more vividly dead-input points, the mechanism becomes locked 
and the mechanical advantage reduces to zero $[12,13]$. Singularity points of a mechanism are dependent on the choice of driving link and must be avoided when attempting to smoothly operate the mechanism. Chase and Mirth [5] defined the regions on a circuit between singularity points as branches. For single-DOF linkages, singularity points are the input limits for that branch. If a GI resides on a motion curve segment (i.e., circuit) that has no singularities, it can be driven with a fully rotatable crank.

As the design parameter changes, the number of branches and their connections, in short the topology of the motion curve, may change at certain critical points. As the design parameter changes, the singularity points sweep out a curve called the singularity curve, and the critical points are the singularities in this curve with respect to the design parameter. The critical points have succinct geometric interpretations as transition linkages. As the singularity is projected onto the actuated and design parameter axes, it forms a singularity trace. It is noted that the number of GIs changes by two as the singularity trace is crossed and number of circuits changes at a critical point. Murray et al. [14] identified relationships of link lengths that defines a transition linkage as the border between topologies. Myszka et al. [15] observed that critical points appearing as smooth extrema on the singularity trace are transition linkages. It was also observed that the Stephenson III linkage and double butterfly linkage have the property that the input angle is able to rotate more than one revolution between singularities and this property is associated with critical points that appear as cusps on the singularity trace.

Nonsingular motion between two GIs is well-known for multi-DOF mechanisms. For example, Innocenti and Parenti-Castelli [16] identified a case of a three-DOF, 3-RPR planar parallel manipulator that could be operated with two prismatic actuators and moved between two GIs without encountering a singularity. McAree and Daniel [17] displayed the motion curve of the 3-RPR platform on a plot showing one output variable (platform angle) and two inputs, which they termed 
the reduced configuration space. They located the input combinations that produce singularity configurations of the 3-RPR and traced that curve onto the reduced configuration space. Wenger et al., $[18,19]$ observed that the projection of the singularity trace divided the reduced configuration space into regions with the same number of GIs. Additionally, they identified that input motions that encircle cusps of the singularity projection produce a "net-zero" activation sequence that places the platform in an alternative GI. Zein et al., [20, 21] enhanced the 3-RPR analysis by projecting the singularity trace onto a two-dimensional plot of the two active input lengths. Macho et al. [22] also used this singularity trace to show a general condition for singularity-free, "net-zero" activation sequences that place the platform into a different GI. In these multi-DOF mechanisms,the singularity-free motion between GIs can occur for arbitrarily small loops in the input space that encircle a cusp.

The analysis methods used for the 3-RPR platform are related to the methods presented here for single-DOF linkages with a designated design parameter. Similar to the singularity projections of $[20,21,22]$, projection of the critical curve into the plane of the input joint angle and the design parameter creates a two-dimensional plot termed the singularity trace. As with the 3-RPR platform, the singularity trace of a single-DOF linkage distinguishes zones having the same number of GIs. If the singularity trace is regarded as a function of the input angle, critical points exist at smooth extrema, at cusps, or at isolated critical points (although these are atypical). The smooth extrema and some cusps are observed to mark values of the design parameter where the number of circuits changes, while the remaining cusps mark values where the number of branches changes without changing the number of circuits. At the latter type of cusp, it is possible for a circuit with an infinitely rotatable input crank to transmute into one where the crank turns greater than one full rotation while moving from one GI to another, that is, one where the motion curve has two GIs on the same branch. 
This thesis illustrates this general analysis methodology including generating motion curves, determining singular positions and critical points, and constructing the singularity trace for planar linkages such as the Watt II, Stephenson III and double butterfly, and spatial linkages such as the spherical four-bar and RCCC linkages. The remainder of the thesis is organized as follows. Chapter 2 introduces the kinematics background necessary for this thesis. Chapter 3 illustrates the derivation process of singularity points, motion curve, critical points, singularity trace and design surface. Chapter 4 provides different linkage examples with the detailed analysis process. Chapter 5 makes conclusions and illustrates future work. 


\section{CHAPTER II}

\section{KINEMATICS BACKGROUND}

The analysis method applied in this thesis utilizes isotropic coordinates to construct loop equations of mathematical models for planar mechanisms. To model spherical and spatial linkages, rotation matrices and homogeneous transforms are used. In this chapter, we introduce the background relating to constructing loop equations, the geometric analysis of singularities, the concept of transition linkages, and notation useful to planar, rotation, and spatial linkages.

\subsection{Loop Closure: Component Method}

Consider a planar 4-revolute (4R) closed chain mechanism as shown in Fig. 2.1. The input link has length $a_{1}$, and the output link has length $a_{3}$. As shown in Fig. 2.1, assume the fixed and moving pivots of the input link to be $\mathbf{O}$ and $\mathbf{A}$, respectively, and the fixed and moving pivots of the output link to be $\mathbf{C}$ and $\mathbf{B}$. The distances between these points are:

$$
a_{1}=|\mathbf{A}-\mathbf{O}|, a_{2}=|\mathbf{B}-\mathbf{A}|, a_{3}=|\mathbf{B}-\mathbf{C}|, a_{4}=|\mathbf{C}-\mathbf{O}|
$$

To analyze the linkage, locate the origin of the fixed frame $F$ at $\mathbf{O}$. Let $\theta_{1}$ be the input angle measured around $\mathbf{O}$ from the $x$-axis of $F$ to $\mathbf{O A}$. Similarly, let $\theta_{3}$ be the angular position of the output link CB.

The relationship between the input angle $\theta_{1}$ of the driving link and the angle $\theta_{3}$ of the driven link can be derived from the condition that $\mathbf{A}$ and $\mathbf{B}$ remain a fixed distance apart throughout the 


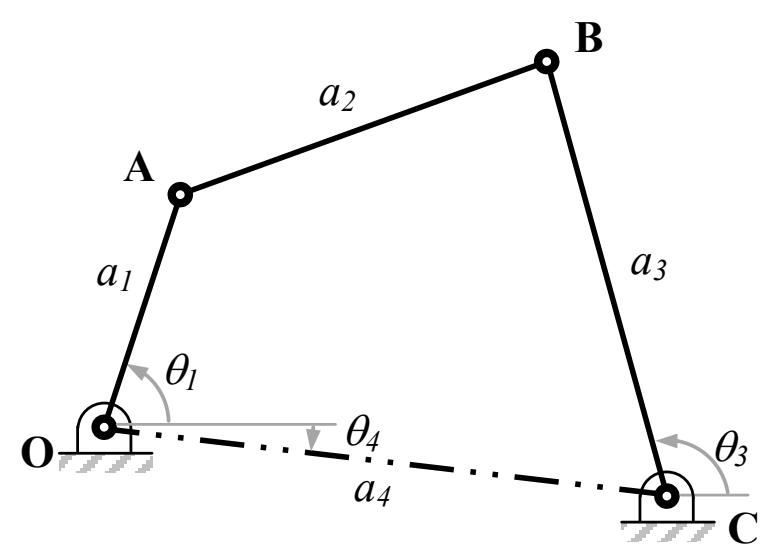

Figure 2.1: A 4R linkage.

motion of the linkage. Since $a_{2}=|\mathbf{B}-\mathbf{A}|$ is constant, the constraint equation can be generated as

$$
f=(\mathbf{B}-\mathbf{A})(\mathbf{B}-\mathbf{A})-a_{2}^{2}=0 .
$$

The coordinates of $\mathbf{A}$ and $\mathbf{B}$ in $F$ are given by

$$
\mathbf{A}=\left\{\begin{array}{l}
a_{1} \cos \theta_{1} \\
a_{1} \sin \theta_{1}
\end{array}\right\} \quad \text { and } \quad \mathbf{B}=\left\{\begin{array}{l}
a_{4} \cos \theta_{4}+a_{3} \cos \theta_{3} \\
a_{4} \sin \theta_{4}+a_{3} \sin \theta_{3}
\end{array}\right\}
$$

Substitute Eq. (2.3) into Eq. (2.2) to obtain

$$
f=f_{1}\left(\theta_{1}\right) \cos \theta_{3}+f_{2}\left(\theta_{1}\right) \sin \theta_{3}+f_{3}\left(\theta_{1}\right)=0 .
$$

where

$$
\begin{gathered}
f_{1}=2 a_{3} a_{4} \cos \theta_{4}-2 a_{1} a_{3} \cos \theta_{1}, \\
f_{2}=2 a_{3} a_{4} \sin \theta_{4}-2 a_{1} a_{3} \sin \theta_{1}, \\
f_{3}=a_{1}^{2}-a_{2}^{2}+a_{3}^{2}+a_{4}^{2}-2 a_{1} a_{4} \cos \left(\theta_{1}-\theta_{4}\right) .
\end{gathered}
$$




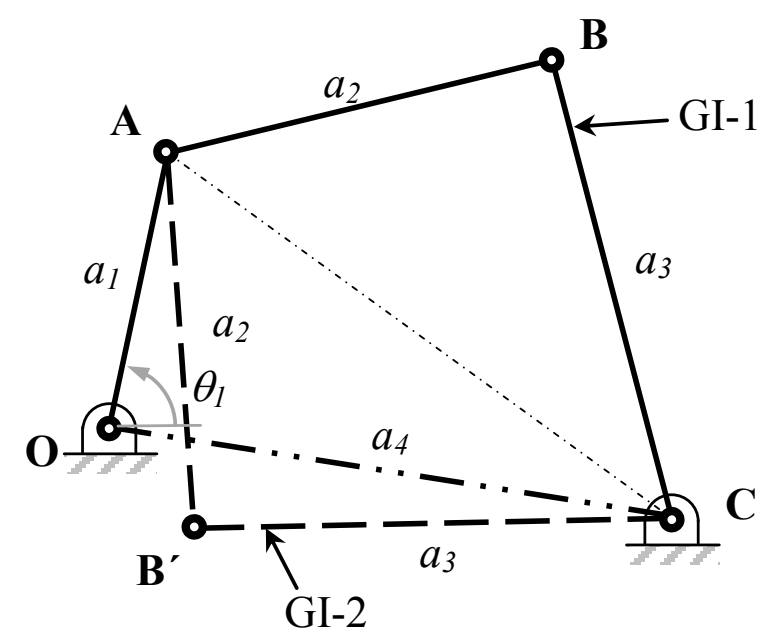

Figure 2.2: A 4R linkage with two different GIs.

The solution to Eq. (2.4) is

$$
\theta_{3}\left(\theta_{1}\right)=\arctan \frac{f_{2}}{f_{1}} \pm \arccos \frac{f_{3}}{\sqrt{f_{1}^{2}+f_{2}^{2}}}
$$

As generated from the Eq. (2.8), there are two angles $\theta_{3}$ for each angle $\theta_{1}$. This arises because the moving pivot $\mathbf{B}$ of the output link can be assembled above or below the diagonal joining the moving pivot $\mathbf{A}$ of the input link to the fixed pivot $\mathbf{C}$ of the output link, and leads to two different GIs as shown in Fig. 2.2. Note that a four-quadrant arctan should be used in Eq. (2.8).

The argument of the arccosine function must be in the range -1 to 1 , which places a solvability constraint on the coefficients $f_{1}, f_{2}$ and $f_{3}$. For a solution to exist,

$$
f_{1}^{2}+f_{2}^{2}-f_{3}^{2} \geq 0
$$

If this constraint is not satisfied, then the linkage cannot be assembled for the specified input link angle $\theta_{1}$. See McCathy and Soh [23] for additional details of this analysis. 


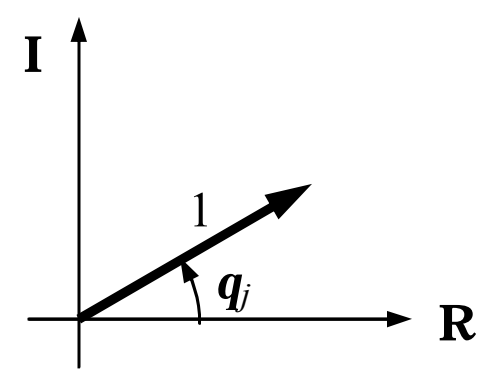

Figure 2.3: A unit vector plotted on the complex plane.

\subsection{Loop Closure: Exponent Method}

An alternate approach to the method of Sec. 2.1 is to model the links as vectors in the complex plane [3]. A unit vector defined by $\theta_{j}$ and shown in Fig 2.3 can be represented in polar form as

$$
\mathbf{T}_{j}=e^{i \theta_{j}}
$$

where $i=\sqrt{-1}$. Subsequently, a binary mechanism link is represented as $a_{j} \mathbf{T}_{j}$, where $a_{j} \in \mathbb{R}$ is the length of the link. Notice that the vector product in the complex plane can be obtained by adding the exponent parts together as

$$
\mathbf{T}_{j} \mathbf{T}_{k}=e^{i \theta_{j}} e^{i \theta_{k}}=e^{i\left(\theta_{j}+\theta_{k}\right)}
$$

This is particularly useful for ternary links that are defined with two joint angles. For the four-bar linkage shown in Fig. 2.1, with redefined link angles as shown in Fig. 2.4, the loop closure equation using complex vectors is

$$
f=a_{1} e^{i \theta_{1}}+a_{2} e^{i \theta_{2}}+a_{3} e^{i \theta_{3}}+a_{4} e^{i \theta_{4}}=0 .
$$

Applying the Euler identity, the components of the unit vector in complex plane can be written as

$$
\mathbf{T}_{j}=\cos \theta_{j}+i \sin \theta_{j}
$$




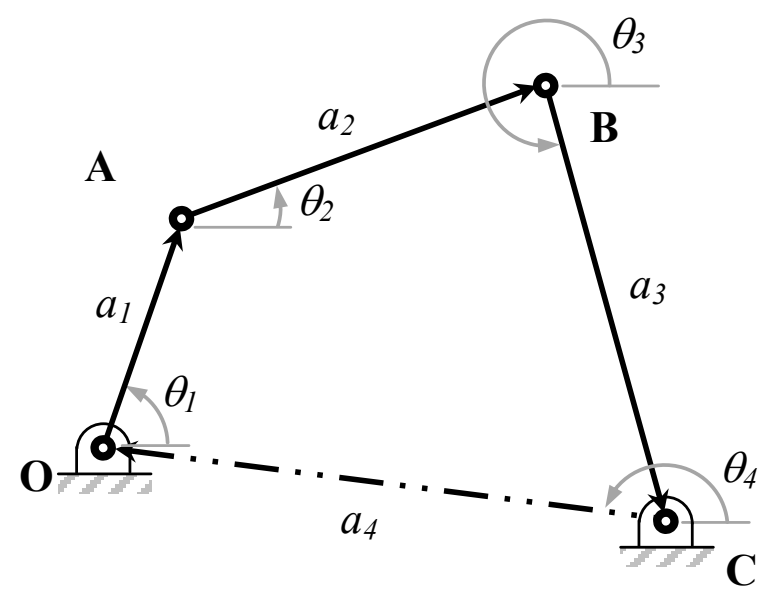

Figure 2.4: A 4R linkage in complex plane.

Thus, Eq. (2.12) can be divided into two parts as

$$
f_{r}=a_{1} \cos \theta_{1}+a_{2} \cos \theta_{2}+a_{3} \cos \theta_{3}+a_{4} \cos \theta_{4}=0,
$$

and

$$
f_{i}=i\left(a_{1} \sin \theta_{1}+a_{2} \sin \theta_{2}+a_{3} \sin \theta_{3}+a_{4} \sin \theta_{4}\right)=0 .
$$

Note that the $i$ may be readily eliminated in Eq. (2.15). By isolating the $a_{2} \cos \theta_{2}$ term in Eq. (2.14) and the $a_{2} \sin \theta_{2}$ in Eq. (2.15), the two expressions may be squared and added resulting in an equation identical to Eqs. (2.4)-(2.7).

\subsection{Isotropic Coordinates}

Isotropic coordinates use the exponential form of the loop equations and their complex conjugates. The complex conjugate of $\mathbf{T}_{j}$ is

$$
\overline{\mathbf{T}}_{j}=e^{-i \theta_{j}}=\cos \theta_{j}-i \sin \theta_{j},
$$


and

$$
\mathbf{T}_{j} \overline{\mathbf{T}}_{j}=e^{i \theta_{j}} e^{-i \theta_{j}}=1
$$

Mechanism links that are represented by exponential variables and their conjugates are isotropic coordinates. A complete discussion of isotropic coordinates and their properties can be found in Wampler [24].

When working in isotropic coordinates the terms "real solution" or "real point" take on an unusual meaning. When we solve a polynomial system whose variables include $\mathbf{T}_{j}$ or $\overline{\mathbf{T}}_{j}$, "real" is taken to mean that the corresponding angle $\theta_{j}$ is real. This implies that $\left|\mathbf{T}_{j}\right|=\left|\overline{\mathbf{T}}_{j}\right|=1$.

The partial derivatives of $\mathbf{T}_{j}$ and $\overline{\mathbf{T}}_{j}$ are

$$
\left(\frac{\partial}{\partial \theta_{j}}\right) \mathbf{T}_{j}=i \mathbf{T}_{j}, \quad\left(\frac{\partial}{\partial \theta_{j}}\right) \overline{\mathbf{T}}_{j}=-i \overline{\mathbf{T}}_{j}
$$

The geometric interpretation of some of the formulas to follow is aided by the following lemma.

Lemma 2.3.1 Let $\mathbf{A}$ and $\mathbf{B}$ be nonzero complex vectors. Then the condition

$$
\mathbf{A} \overline{\mathbf{B}}-\overline{\mathbf{A B}}=0
$$

implies that $\mathbf{A}$ and $\mathbf{B}$ are parallel.

Proof. Multiply through by AB to obtain

$$
\mathbf{A}^{2} \mathbf{B} \overline{\mathbf{B}}-\mathbf{A} \overline{\mathbf{A}} \mathbf{B}^{2}=0
$$

Applying Eq. (2.17), $\mathbf{A} \overline{\mathbf{A}}=a^{2}$ and $\mathbf{B} \overline{\mathbf{B}}=b^{2}$, where $a$ and $b$ are the lengths of $\mathbf{A}$ and $\mathbf{B}$, respectively. So the expression factors as

$$
(b \mathbf{A}+a \mathbf{B})(b \mathbf{A}-a \mathbf{B})=0,
$$

which requires either $(b \mathbf{A}+a \mathbf{B})=0$ or $(b \mathbf{A}-a \mathbf{B})=0$. If $(b \mathbf{A}+a \mathbf{B})=0$ then $\mathbf{A}$ and $\mathbf{B}$ are in opposite directions. If $(b \mathbf{A}-a \mathbf{B})=0$ then $\mathbf{A}$ and $\mathbf{B}$ are in the same direction. In either case, $\mathbf{A}$ and $\mathbf{B}$ are parallel. 


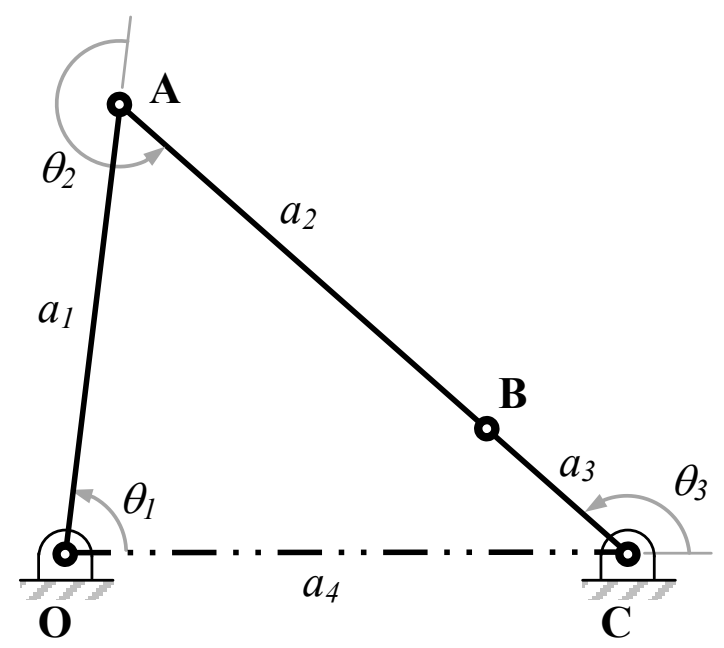

Figure 2.5: a 4R linkage at a singularity.

\subsection{Geometric Analysis of Singularities}

In this thesis, the identification of the actuator singularities of a closed loop linkage is a foundational topic. A closed-loop kinematic chain consists of a set of rigid bodies connected to each other with joints where at least one closed loop exists. The chain is also characterized by a set of inputs, which correspond to the actuated joints, and by a set of output coordinates. These inputs and outputs depend on the nature and purpose of the kinematic chain [13]. A singularity in kinematics refers to configurations in which a mechanism's number of DOF changes instantaneously. This change is reflected by a drop in rank of the Jacobian of the output joint parameters [25]. Physically, if a mechanism of one DOF is in an actuator singularity position, then a torque applied to the input link cannot create motion of the other links of the linkage. Consider Fig. 2.5 where OA is the input link. This $4 \mathrm{R}$ linkage is in a singularity position. A torque applied to $\mathbf{O A}$ in either direction cannot move the linkage and the other driven links $\mathbf{A B}$ and $\mathbf{B C}$ remain stationary. Similarly, as shown in Fig. 2.6, the input link $\mathbf{O A}$ cannot be moved around $\mathbf{O}$. The three pivots $\mathbf{A}, \mathbf{B}$ and $\mathbf{C}$ lie on 


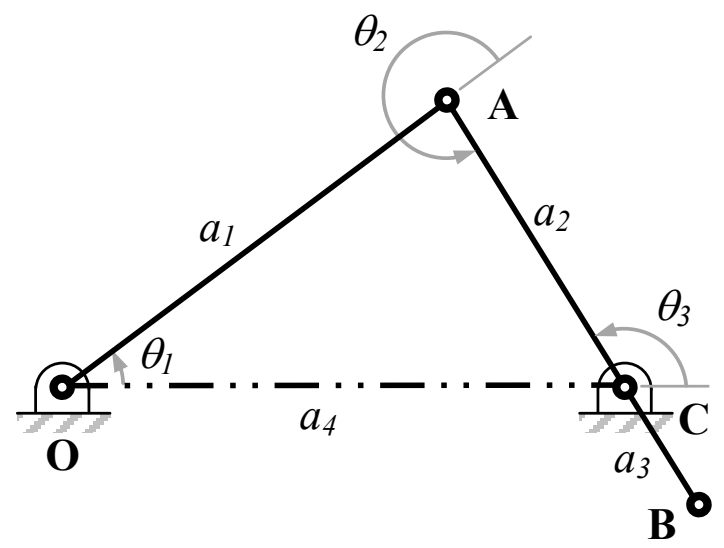

Figure 2.6: The same 4R linkage at another singularity.

the same line in both cases. This observation can be generalized to state that a $4 \mathrm{R}$ mechanism's singularities occur when the driven pivots become collinear [3].

Consider the Stephenson III linkage driven at $\mathbf{A}$ as shown in Fig. 2.7. This mechanism shares the collinear singularity condition within the four-bar loop of ODFI. Additionally, a singularity exists when the three lines that go through $\mathbf{O D}, \mathbf{E F}$ and $\mathbf{C B}$ intersect at one point. The input link AB is not drivable at pivot A. Note that the singularities of ODFI are a subset of the more general case.

However, as mechanisms become increasingly complex, the capacity to observe singularities as simple geometric relationship between pivots diminishes [26]. The method applied in this thesis assesses the rank of the forward kinematics Jacobian to generate singularities of complex mechanisms. 


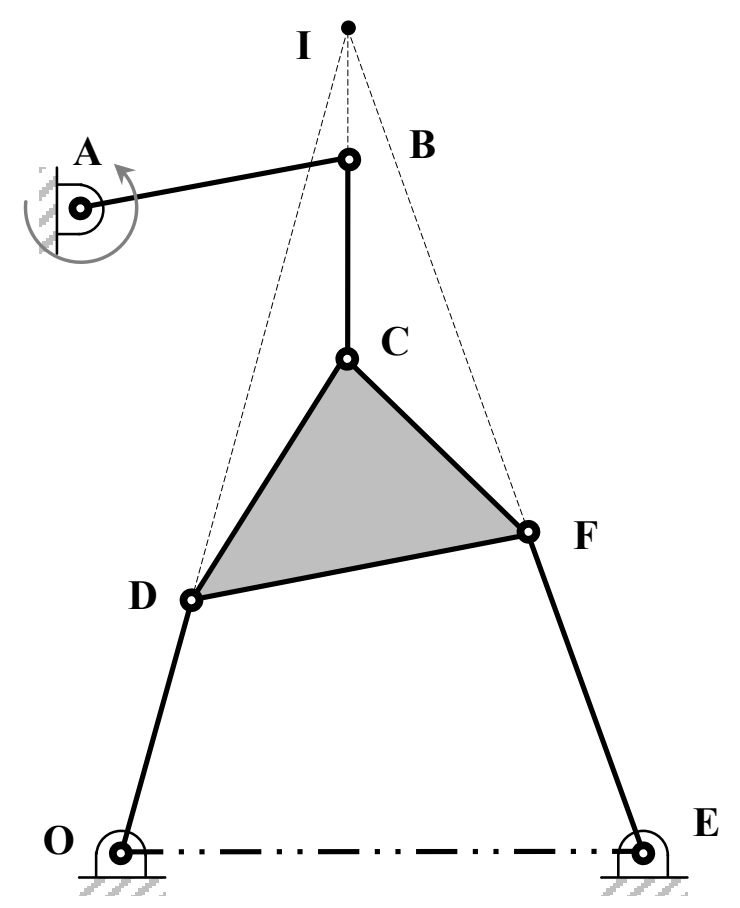

Figure 2.7: A Stephenson III linkage at a singularity.

\subsection{Transition Linkage}

Considering a 4R linkage, assume that $l$ is the longest link, $s$ is the shortest link, and $p$ and $q$ are the remaining two links. The Grashof criterion states that if $l+s<p+q$, this $4 \mathrm{R}$ linkage will have at least one link that will fully rotate [23]. For a Grashof 4R linkage, one which satisfies the inequality, increasing the length of the longest link will create a 4R linkage that will eventually not satisfy the inequality condition and will no longer have a fully rotating link. In order to be physically realizable and able to move, the longest link of this $4 \mathrm{R}$ linkage can be further increased until the condition $l<s+p+q$ becomes false. When $l=s+p+q$, the linkage loses a DOF and becomes a structure. If $l>s+p+q$ the linkage cannot be assembled. The situations in which the link's lengths satisfy $l+s=p+q$ and $l=s+p+q$ define the boundaries at which the behavior 
of the linkage changes. For the case $l+s=p+q$, it defines the boundary between the Grashof and non-Grashof linkages. The boundary condition between physically realizable linkages and those that are not is defined by the case $l=s+p+q$. Both cases are transition linkages. Notice that either 4R transition linkage discussed above has a configuration at which all of the joints of linkage are collinear. Thus, transition linkages are those linkages dimensioned such that they are on the boundary between different types of behavior.

Murray et al. [14] concluded that, for general planar linkages, a transition linkage has a configuration in which the nonsquare matrix defined by the derivative of the loop closure equations with respect to the entire set of joint variables loses rank. This work, which generalizes the earlier work by positing that transition linkages occur at critical points of the singularity trace, finds additional sets of dimensions that define transition linkages. This is most readily displayed in the Watt II linkage where critical points are found on the singularity trace that do not correspond to the nonsquare matrix losing rank. The details are presented in Sec. 4.1.

\subsection{Spatial Kinematics}

As one goal of this thesis is to extend the idea of the singularity trace to spatial linkages, additional mathematical tools are needed. For the planar case, isotropic coordinates prove sufficient for all derivations contained herein. Isotropic coordinates, however, do not readily extend to spatial relationships. The basic kinematic concepts needed for spatial situations arising in this work are briefly reviewed here.

\subsubsection{Rotations}

Define the rotation matrices $[X(\theta)]$ and $[Z(\phi)]$ about the $x$ - and $z$-axes as

$$
[X(\theta)]=\left[\begin{array}{ccc}
1 & 0 & 0 \\
0 & \cos \theta & -\sin \theta \\
0 & \sin \theta & \cos \theta
\end{array}\right],[Z(\theta)]=\left[\begin{array}{ccc}
\cos \phi & -\sin \phi & 0 \\
\sin \phi & \cos \phi & 0 \\
0 & 0 & 1
\end{array}\right] .
$$




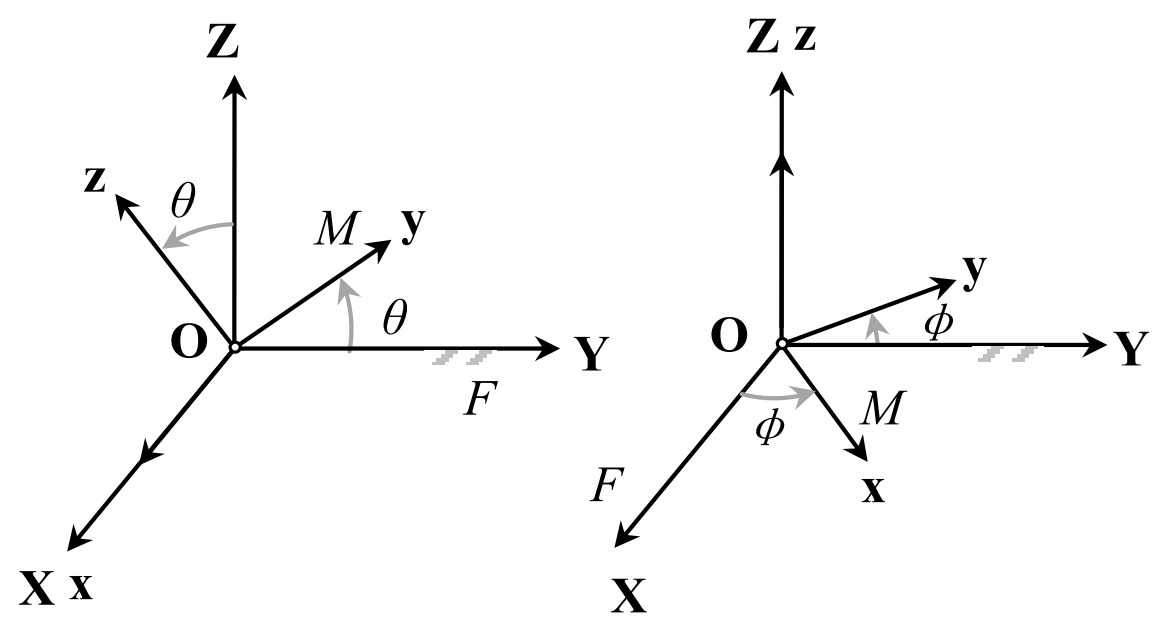

Figure 2.8: The fixed and moving frames defining spatial rotations about the $x$-axis and $z$-axis.

These matrices may be used to relate coordinates shown in the reference frames in Fig. 2.8. Note that the inverse of a rotation matrix is its transpose,

$$
\begin{aligned}
& {[X(\theta)]^{-1}=[X(\theta)]^{T}=\left[\begin{array}{ccc}
1 & 0 & 0 \\
0 & \cos \theta & \sin \theta \\
0 & -\sin \theta & \cos \theta
\end{array}\right],} \\
& {[Z(\theta)]^{-1}=[Z(\theta)]^{T}=\left[\begin{array}{ccc}
\cos \phi & \sin \phi & 0 \\
-\sin \phi & \cos \phi & 0 \\
0 & 0 & 1
\end{array}\right] .}
\end{aligned}
$$

\subsubsection{Spatial Displacements}

As shown in Fig. 2.9, let the moving frame $M$ and the fixed frame $F$ coincide at origin $\mathbf{O}$ of $F$ initially. The displacement of $M$ relative to $F$ consists of a slide $d$ and rotation $\theta$ along and around the $x$-axis of $F$. Construct the $4 \times 4$ homogeneous transform,

$$
\left\{\begin{array}{l}
X \\
Y \\
Z \\
1
\end{array}\right\}=\left[\begin{array}{cccc}
1 & 0 & 0 & d \\
0 & \cos \theta & -\sin \theta & 0 \\
0 & \sin \theta & \cos \theta & 0 \\
0 & 0 & 0 & 1
\end{array}\right]\left\{\begin{array}{l}
x \\
y \\
z \\
1
\end{array}\right\}
$$




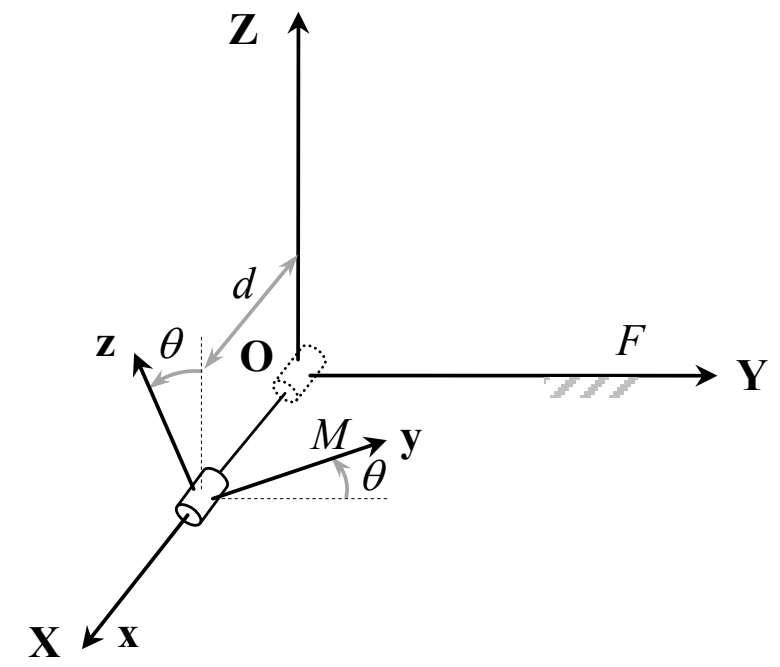

Figure 2.9: Coordinate screw displacement along $x$-axis.

or

$$
\mathbf{V}=[X(\theta, d)] \mathbf{v}
$$

Similarly, the displacement shown in Fig. 2.10 can be written as

$$
\left\{\begin{array}{l}
X \\
Y \\
Z \\
1
\end{array}\right\}=\left[\begin{array}{cccc}
\cos \phi & -\sin \phi & 0 & 0 \\
\sin \phi & \cos \phi & 0 & 0 \\
0 & 0 & 1 & c \\
0 & 0 & 0 & 1
\end{array}\right]\left\{\begin{array}{l}
x \\
y \\
z \\
1
\end{array}\right\},
$$

or

$$
\mathbf{V}=[Z(\phi, c)] \mathbf{v}
$$

The transform $[X(\theta, d)]$ and $[Z(\phi, c)]$ are the coordinate screw displacements about the $x$-axis and the $z$-axis respectively. The screw displacement $[Y(\psi, e)]$ about the $y$-axes can be defined as,

$$
[Y(\psi, e)]=\left[\begin{array}{cccc}
\cos \psi & 0 & \sin \psi & 0 \\
0 & 1 & 0 & e \\
-\sin \psi & 0 & \cos \psi & 0 \\
0 & 0 & 0 & 1
\end{array}\right] .
$$

These coordinate screw displacements are used to formulate the kinematics equations for spatial linkage analysis in this thesis. 


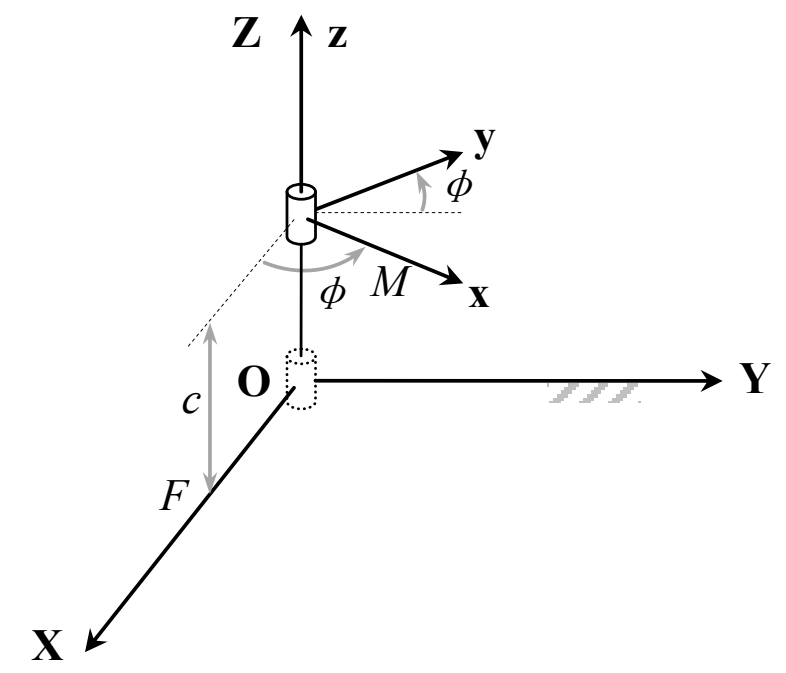

Figure 2.10: Coordinate screw displacement along $z$-axis.

It is useful to note that the inverse of a coordinate screw displacement can be obtained by negating its parameters. For Example,

$$
[Z(\phi, c)]^{-1}=[Z(-\phi,-c)]=\left[\begin{array}{cccc}
\cos \phi & \sin \phi & 0 & 0 \\
-\sin \phi & \cos \phi & 0 & 0 \\
0 & 0 & 1 & -c \\
0 & 0 & 0 & 1
\end{array}\right]
$$

Notice that $[Z(\phi, c)]^{-1}$ is not the transpose of $[Z(\phi, c)]$. 


\section{CHAPTER III}

\section{SINGULARITY POINTS, MOTION CURVE, CRITICAL POINTS, SINGULARITY TRACE AND DESIGN SURFACE}

Consider a general mechanism with a single DOF. The input variable is designated as $x \in \mathbb{C}$. Also, designate a design variable, $p \in \mathbb{C}$, and let $y$ be all the remaining variables $y \in \mathbb{C}^{N}$. We assume that the loop closure equations are formulated as

$$
f(p, x, y)=0, \quad f: \mathbb{C} \times \mathbb{C} \times \mathbb{C}^{N} \rightarrow \mathbb{C}^{N}
$$

For a fixed value of $p$, the mechanism has a single DOF motion represented by a motion curve $\mathcal{C}_{p} \subset \mathbb{C}^{N+1}, \operatorname{dim}\left(\mathcal{C}_{p}\right)=1$

The exponential form of the loop closure conditions for any $n$-link $\ell$-loop planar mechanism with $\mathrm{R}$ joints can be written as

$$
f_{k}=\sum_{j=1}^{n} a_{j k} e^{i \theta_{j}}=0, \quad k=1, \ldots, \ell
$$

The coefficient $a_{j k} \in \mathbb{C}$, is the edge of link $j$ that connects joints in loop $k$. For a binary link, there is just one edge, and it can be taken as a real number, its length. Ternary links (triangles) and higher will have different edges contributing to different loops and the associated coefficients are then complex. See [27] and Sec. 4.1. 
The $\ell$ loop equations in the exponential form of Eq. (3.2) convert to $2 \ell+n-m$ equations in isotropic coordinates as:

$$
\begin{aligned}
& g_{k}=\sum_{j=1}^{n} a_{j k} \mathbf{T}_{j}=0, \bar{g}_{k}=\sum_{j=1}^{n} a_{j k}^{\prime} \overline{\mathbf{T}}_{j}=0, \quad k=1, \ldots, \ell \\
& h_{j}=\mathbf{T}_{j} \overline{\mathbf{T}}_{j}-1=0, \quad j=m+1, \ldots, n .
\end{aligned}
$$

Note that in the conjugate loop equations, $\bar{g}_{k}$, the complex conjugates, $a_{j k}^{\prime}$, of the link edges appear. Equations $\mathbf{T}_{j} \overline{\mathbf{T}}_{j}-1=0, j=1, \ldots, m$ are not included in the equation set, because link 1 is assumed to be driving link and link $2, \ldots$, link $\mathrm{m}$ are assumed to be the (grounded) fixed links, so their angles $\theta_{1}, \theta_{2}, \ldots, \theta_{m}$ are known.

Of the various choices for formulating loop closure equations, two closely related ones are used in this thesis where both model links as vectors in the complex plane. As an illustration, consider the four-bar linkage as shown in Fig. 2.4. Designate the input as angle $x=\theta_{1}$ and the design parameter as $p=a_{1}$. The remaining variables are $y=\left\{\theta_{2}, \theta_{3}\right\}$, while $\theta_{4}$ and $a_{2}, a_{3}, a_{4}$ are taken as given. The four-bar has a single loop, whose closure condition in complex exponential notation is given as

$$
f=a_{1} e^{i \theta_{1}}+a_{2} e^{i \theta_{2}}+a_{3} e^{i \theta_{3}}+a_{4} e^{i \theta_{4}}=0 .
$$

With $a_{1}$ fixed, the real and imaginary parts of this complex equation impose two constraints on $\left\{\theta_{1}, \theta_{2}, \theta_{3}\right\}$, resulting in a motion curve as illustrated in Fig. 3.1.

An alternative to the exponential form is to convert Eq. (3.5) to its equivalent isotropic form as

$$
\begin{aligned}
& g=a_{1} \mathbf{T}_{1}+a_{2} \mathbf{T}_{2}+a_{3} \mathbf{T}_{3}+a_{4} \mathbf{T}_{4}=0, \\
& \bar{g}=a_{1} \overline{\mathbf{T}}_{1}+a_{2} \overline{\mathbf{T}}_{2}+a_{3} \overline{\mathbf{T}}_{3}+a_{4} \overline{\mathbf{T}}_{4}=0, \\
& h_{1}=\mathbf{T}_{1} \overline{\mathbf{T}}_{1}-1=0, \\
& h_{2}=\mathbf{T}_{2} \overline{\mathbf{T}}_{2}-1=0, \\
& h_{3}=\mathbf{T}_{3} \overline{\mathbf{T}}_{3}-1=0 .
\end{aligned}
$$




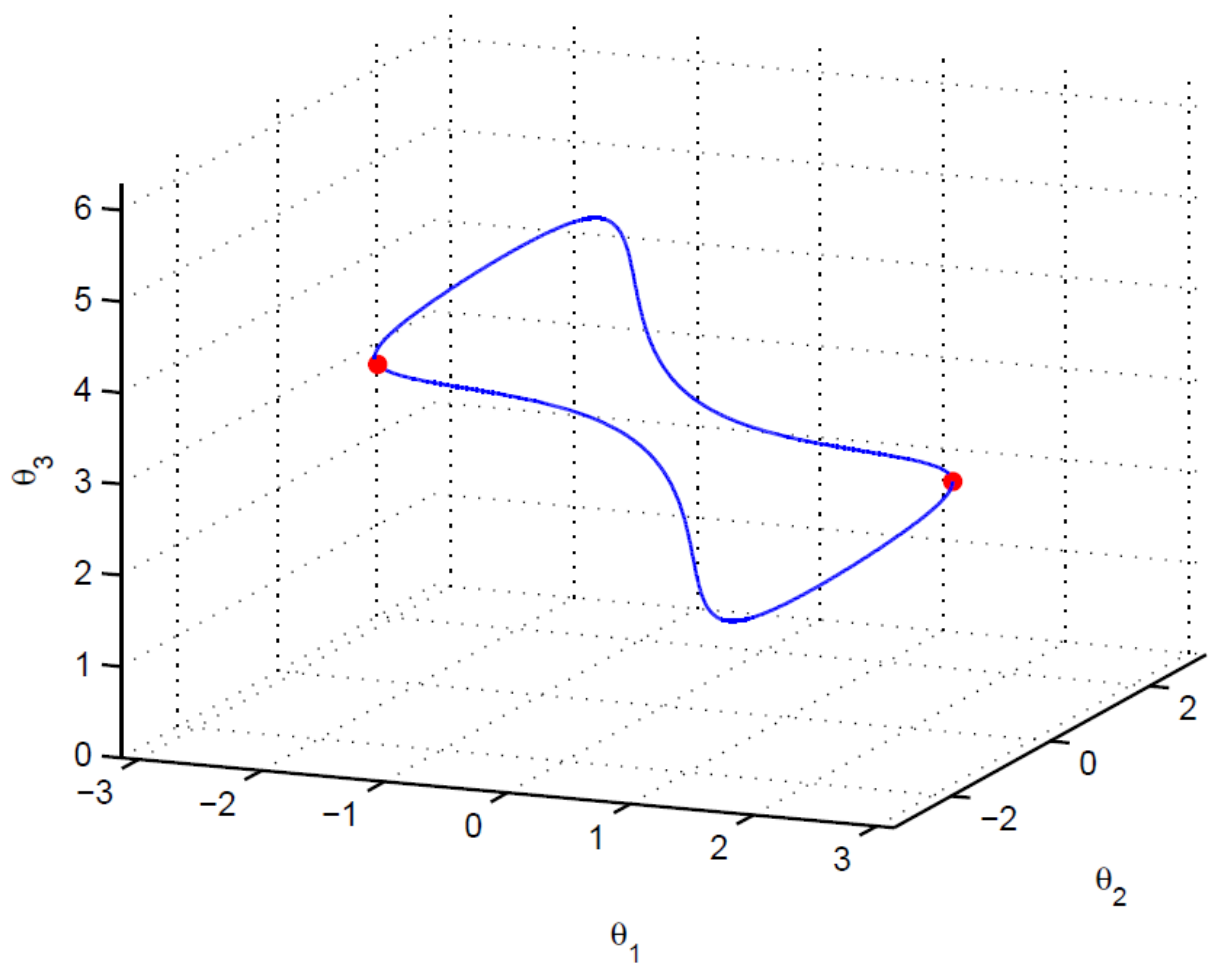

Figure 3.1: The motion curve for a four-bar mechanism as expressed in Eq. 3.5 or 3.6.

With $a_{1}$ fixed, this system of five equations describes a curve in the six-dimensional space of $\left\{\mathbf{T}_{1}, \mathbf{T}_{2}, \mathbf{T}_{3}, \overline{\mathbf{T}}_{1}, \overline{\mathbf{T}}_{2}, \overline{\mathbf{T}}_{3}\right\}$. The points of this curve are in one-to-one correspondence with the points of the curve described by Eq. (3.5), and we may think of them as the same four-bar motion curve. The isotropic formulation has the convenient property that all its equations are polynomial, which facilitates finding solutions, while the complex exponential formulation is more compact. We will move fluidly between the two approaches as convenient below. 


\subsection{Forward Kinematics}

In the case of the four-bar, where $\theta_{1}$ is designated as the input, $h_{1}$ is dropped from Eq. (3.3). Next, the loop equations, $g_{k}$, and the conjugate loop equations, $\bar{g}_{k}$ are all linear, so these may be used to eliminate some variables. For example, for the four-bar of Eq. (3.6), the equations $g=0$ and $\bar{g}=0$ lead to defining the expressions

$$
\begin{aligned}
& \mathbf{R}_{3}:=a_{1} \mathbf{T}_{1}+a_{2} \mathbf{T}_{2}+a_{4} \mathbf{T}_{4} \\
& \overline{\mathbf{R}}_{3}:=a_{1} \overline{\mathbf{T}}_{1}+a_{2} \overline{\mathbf{T}}_{2}+a_{4} \overline{\mathbf{T}}_{4},
\end{aligned}
$$

so that $\mathbf{R}_{3}=-a_{3} \mathbf{T}_{3}$ and $\overline{\mathbf{R}}_{3}=-a_{3} \overline{\mathbf{T}}_{3}$. Accordingly, for $a_{3} \neq 0$, we may form $a_{3}^{2} h_{3}=0$ to get

$$
H_{3}:=\mathbf{R}_{3} \overline{\mathbf{R}}_{3}-a_{3}^{2}=0
$$

This equation along with $h_{2}=\mathbf{T}_{2} \overline{\mathbf{T}}_{2}-1=0$ makes a system of two equations for $\left\{\mathbf{T}_{2}, \overline{\mathbf{T}}_{2}\right\}$. This is the form of the equations that will be most useful later in the paper. These two polynomials can be solved many ways: one is to use $h_{2}=0$ to write $\overline{\mathbf{T}}_{2}=1 / \mathbf{T}_{2}$, which after substitution into Eq. (3.8), gives a quadratic equation for $\mathbf{T}_{2}$.

In $[27,28]$, one can find recipes for solving general planar mechanisms formulated in isotropic coordinates as in Eq. (3.3). Similar to the derivation just discussed for the four-bar, the recipe in [28] eliminates half of the variables using the loop equations and then solves the system of bilinear polynomials using the Dixon determinant. (See [29] for a related method based on the Dixon determinant but using tangent-half-angle substitutions.)

\subsection{Find all Singularity Points}

Given a mechanism (i.e., a fixed design parameter $p$ ), it is desirable to find all branches of the motion with respect to the designated input parameter, $x$. As illustrated in Figure 3.2 and described in [5], the branches meet at the turning points of the curve, where the mechanism moves 
differentially without any motion at the input. The mechanism is locked at a turning point (i.e., singular position), but can be driven smoothly from the input along each motion branch.

For fixed $p$, the tangent $[\Delta x, \Delta y]$ to the motion curve is given by

$$
f_{x} \Delta x+f_{y} \Delta y=0
$$

where $f_{x}=\frac{\partial f}{\partial x} \in \mathbb{C}^{N \times 1}$ and $f_{y}=\frac{\partial f}{\partial y} \in \mathbb{C}^{N \times N}$. The turning points occur when $\Delta y \neq 0$ with $\Delta x=0$, which implies that

$$
D(p, x, y):=\operatorname{det} f_{y}=0 \text {. }
$$

Accordingly, the turning points are solutions to the system of equations

$$
F(p, x, y)=\left[\begin{array}{c}
f(p, x, y) \\
D(p, x, y)
\end{array}\right]=0
$$

For given $p$, this is a system of $N+1$ equations in $N+1$ unknowns which can be solved to find the turning points. An alternative formulation that avoids forming the determinant is to solve for tangent vectors that have no $x$-component, that is, randomly choose a $1 \times N$ vector $v$ and solve the system

$$
\hat{F}(p, x, y, \Delta y)=\left[\begin{array}{c}
f(p, x, y) \\
f_{y} \Delta y \\
v \cdot \Delta y-1
\end{array}\right]=0 .
$$

Note that if $\Delta y$ is a solution to $f_{y} \Delta y=0$, then so is $\alpha \Delta y$ for any scalar $\alpha$. The randomly chosen $v$ picks out a unique scaling of the tangent vector. The final expression $(v \cdot \Delta y-1=0)$ ensures $\Delta y \neq 0$. Since higher-dimensional tangent spaces are possible, one must search for these when solving Eq. (3.12). Methods for doing so are discussed in [30, Part II] and are available in the Bertini software package [2].

The four-bar example is now used to illustrate. The loop closure conditions are $\left\{h_{1}, h_{2}, H_{3}\right\}=$ 0 from Eqs. (3.6) and (3.8). Using Eq. (2.18), the partial derivatives of $h_{1}$ are

$$
\frac{\partial h_{1}}{\partial \theta_{1}}=i \mathbf{T}_{1} \overline{\mathbf{T}}_{1}-i \mathbf{T}_{1} \overline{\mathbf{T}}_{1} \equiv 0, \quad \text { and } \quad \frac{\partial h_{1}}{\partial \theta_{2}} \equiv 0,
$$



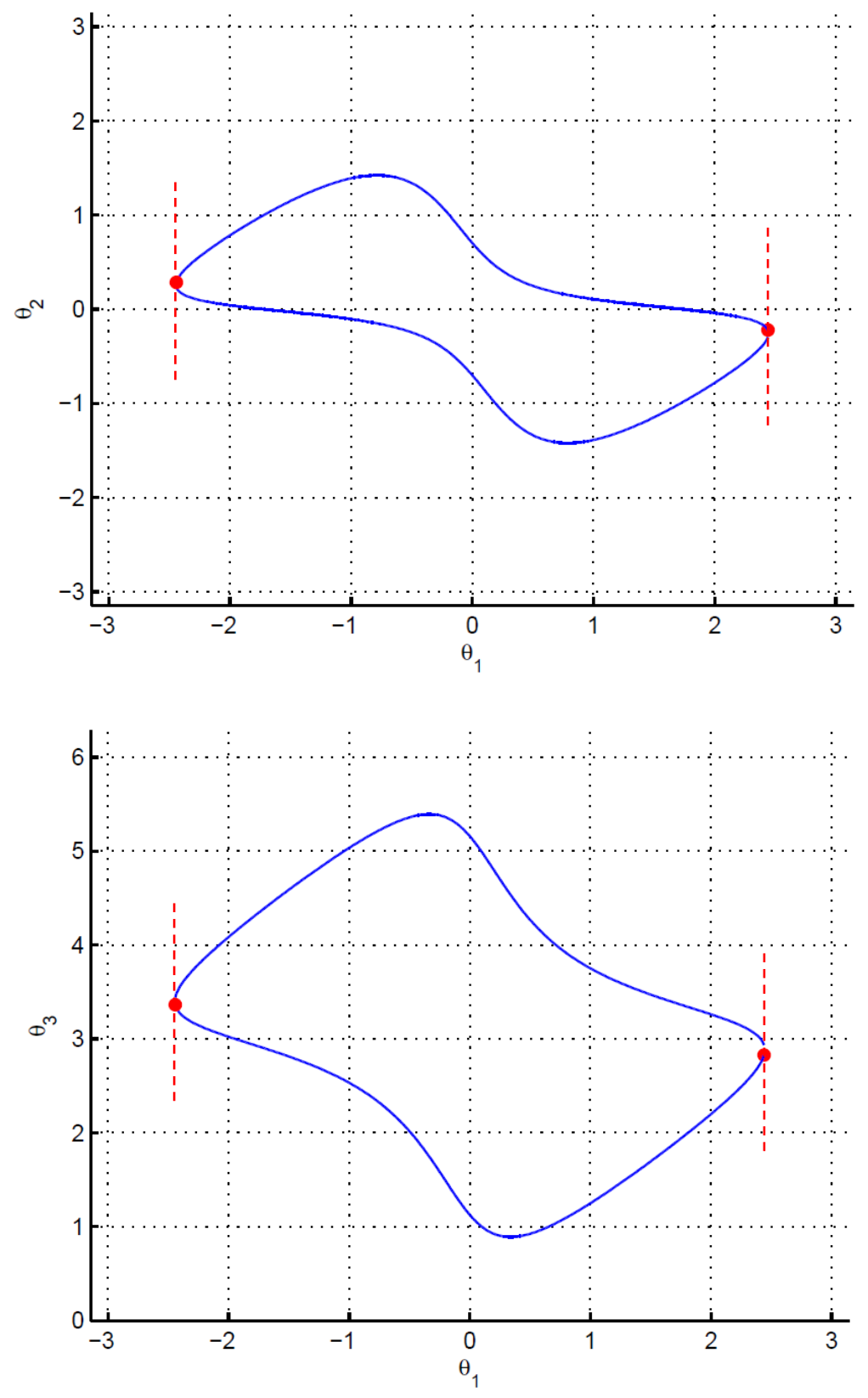

Figure 3.2: Projections of the motion curve from Fig. 3.1. Turning points, shown with red markers, have no $x\left(\theta_{2}\right.$ in this case $)$ component. 
and similarly for $h_{2}$, so the only nontrival partial derivatives are those for $H_{3}$ :

$$
\begin{gathered}
f_{x}=\frac{\partial f}{\partial \theta_{1}}=i a_{1}\left(\mathbf{T}_{1} \overline{\mathbf{R}}_{3}-\mathbf{R}_{3} \overline{\mathbf{T}}_{1}\right), \\
f_{y}=\frac{\partial f}{\partial \theta_{1}}=i a_{2}\left(\mathbf{T}_{2} \overline{\mathbf{R}}_{3}-\mathbf{R}_{3} \overline{\mathbf{T}}_{2}\right) .
\end{gathered}
$$

Since $f_{y}$ is $1 \times 1$ in this instance, its determinant is itself. We may cancel the common factor of $i a_{3}$ and write the system that determines the turning points as

$$
F=\left[\begin{array}{c}
\mathbf{T}_{1} \overline{\mathbf{T}}_{1}-1 \\
\mathbf{T}_{2} \overline{\mathbf{T}}_{2}-1 \\
\mathbf{R}_{3} \overline{\mathbf{R}}_{3}-a_{3}^{2} \\
\mathbf{T}_{2} \overline{\mathbf{R}}_{3}-\overline{\mathbf{T}}_{2} \mathbf{R}_{3}
\end{array}\right]=0
$$

This is a polynomial system of four equations in $\left\{\mathbf{T}_{1}, \mathbf{T}_{2}, \overline{\mathbf{T}}_{1}, \overline{\mathbf{T}}_{2}\right\}$. As a bilinear system in $\left\{\mathbf{T}_{2}, \mathbf{T}_{3}\right\},\left\{\overline{\mathbf{T}}_{2}, \overline{\mathbf{T}}_{3}\right\}$, it has at most six solutions [30, Sec. 8.4.2], but it can be seen that two of these go to infinity, leaving just four finite roots.

By Lemma 2.3.1, we see that the turning point condition implies that links 3 and 4 are parallel. This is the well-known dead-input condition for a four-bar. With this geometric interpretation, one may find the turning points by solving first a triangle with sides $a_{1}, a_{4},\left(a_{2}+a_{3}\right)$ and then one with sides $a_{1}, a_{4},\left|a_{2}-a_{3}\right|$. One gets two values for $\theta_{1}$ from each of these, for a total of four, in agreement with the bilinear analysis.

For the four-bar with particular values $\left\{a_{1}, a_{2}, a_{3}, a_{4}\right\}=\{0.6,0.88,0.63,1\}$ and $\theta_{4}=0$, there are two real solutions, $\left(\theta_{1}, \theta_{2}\right)=( \pm 0.6970, \mp 2.8837)$. These are shown as red dots on the motion curve in Fig. 3.1.

\subsection{Find all Branches}

The complete motion curve may be generated by solving the forward kinematics for a finelysampled set of inputs, $x$. As this potentially gives many solution points at each $x$, there remains the task of connecting the points together to form approximations of the branches. Also, to get 
a uniformly smooth representation of the branches, densely sampling near the turning points is needed.

A simpler approach, described in [31] and summarized in [32], gives an algorithm for finding the real points in a complex curve. Essentially, the method is as follows:

1. Find all real turning points (see Sec. 3.2) and sort them in ascending input values.

2. Let $t_{1}<t_{2}<\cdots<t_{m}$ be the input values at the turning points from Step 1. If there are no turning points, set $t_{1}=0$.

3. For one input value $x_{1}<t_{1}$ and one value $x_{j}, j=2, \ldots, m$ in each interval $t_{j-1}<x_{j}<t_{j}$, solve the forward kinematics (see Sec. 3.1).

4. Trace the mechanism motion arcs from each real solution from Step 3 to its neighboring turning points. As we assume the input is an angle, this means that solutions at $x_{1}$ also trace backwards around to $t_{m}$.

5. (Optional) Merge together any arcs from Step 4 that meet at a regular point, i.e., not at a turning point.

This process ends with a list of the branches and a numerical sampling of points along each branch. For the purpose of merely plotting the motion curve, skip the final merging step, but then any one branch may be filled in by several arcs. The merging step is necessary if we want to determine exactly the number of branches and the full extent of each.

There are several ways to trace the motion arcs as required in Step 4. As software for solving ordinary differential equations (ODEs) is readily available, it can be convenient to convert Eq. (3.9) to the differential equation

$$
f_{x}+f_{y}(d y / d x)=0
$$


Step 3 provides the initial conditions for $(x, y)$ and the neighboring turning points provide the ending value of $x$. An alternative is to use a prediction-correction path-tracking method [33]. In essence, these intersperse ODE methods for advancing along the path (prediction) with the NewtonRaphson method at the current value of $x$. The Newton-Raphson procedure is the correction phase of the prediction-correction cycle, serving to eliminate any accumulation of integration error from the prediction phase.

Note that in Step 2, the absence of any turning points means that either there are no branches at all (the mechanism cannot be assembled) or the input is a crank for all the branches. For the empty set of turning values, set $t_{1}=0$ so that Step 3 is cued to discover if any branches exist.

\subsection{Find Critical Points}

In generating the motion curve and finding the singularities, the design parameter $p$ was always given. Now consider how the behavior of the mechanism changes as the design parameter varies. Consequently, consider that with $p$ varying, the system Eq. (3.11) becomes $N+1$ variables in $N+2$ unknowns and defines a curve, say $\mathcal{K} \subset \mathbb{C}^{N+2}, \operatorname{dim}(\mathcal{K})=1$. Since this consists of the turning points as $p$ varies, we call $\mathcal{K}$ the singularity curve.

To generate the singularity trace as $p$ varies, carry out the corresponding algorithm in Steps 1-5, where the generation of a motion curve is described. The analogous process will divide $\mathcal{K}$ into branches, each of which continues monotonically with parameter $p$. The turning points with respect to $p$ of the singularity trace are called the critical points.

The importance of the critical points is that they mark values of the design parameter where the number of branches in the motion curve can change. Murray [14] describes the resulting mechanism at the critical points as "transition linkages." As the design parameter varies, a pair of branches may disappear where two real turning points meet then become complex, or vice versa, two complex turning points can meet and become real to create a pair of new branches. While this is the typical 
behavior, other phenomena are also possible. A singular critical point (for example, a double root of the critical point conditions) might be isolated in the reals and correspond to a degeneracy that exists just at one parameter value. (For example, the motion curve might pinch to touch itself at one value of $p$ and immediately separate on either side.) Another possibility is that the singularity trace might cross itself, with some kind of degeneracy occurring at the crossing point.

To find the critical points, we proceed analogously to finding singular points, replacing $x$ by $p$, $y$ by $(x, y)$, and $f$ by $F$. The tangency condition Eq. (3.9) becomes

$$
\frac{\partial F}{\partial p} \Delta p+\frac{\partial F}{\partial x} \Delta x+\frac{\partial F}{\partial y} \Delta y=0
$$

Defining $F_{x y}=\left[\begin{array}{ll}\frac{\partial F}{\partial x} & \frac{\partial F}{\partial y}\end{array}\right]$, the condition for the critical points, analogous to Eq. (3.11), is

$$
\left[\begin{array}{c}
F \\
\operatorname{det} F_{x y}
\end{array}\right]=\left[\begin{array}{c}
f \\
\operatorname{det} f_{y} \\
\operatorname{det} F_{x y}
\end{array}\right]=0
$$

It is useful to note that

$$
F_{x y}=\left[\begin{array}{cc}
f_{x} & f_{y} \\
\frac{\partial \operatorname{det} f_{y}}{\partial x} & \frac{\partial \operatorname{det} f_{y}}{\partial y}
\end{array}\right] .
$$

As with turning points, an alternative formulation that avoids forming the determinants is to solve for a tangent vectors that have no $x$-component and tangent vectors that have no $x$-component or $y$-component. That is, randomly choose a $1 \times N$ vectors $v$ and $w$ and solve the system

$$
\hat{F}(p, x, y, \Delta x, \Delta y)=\left[\begin{array}{c}
f(p, x, y) \\
f_{y} \Delta y \\
v \cdot \Delta y-1 \\
F_{x y}[\Delta x \Delta y]^{T} \\
w \cdot\left[\begin{array}{ll}
\Delta x & \Delta y
\end{array}\right]^{T}-1
\end{array}\right]=0 .
$$

Again, as in the case of Eq. (3.12), one must check for the possible existence of solutions where the Jacobian matrices $f_{y}$ and $F_{x y}$ lose extra rank so that the associated tangent spaces are higherdimensional.

As in the preceding problems, the four-bar is used to illustrate. To form Eq. (3.11),

$$
F=\left[\begin{array}{c}
f \\
D
\end{array}\right]
$$


with $f=\left\{h_{1}, h_{2}, H_{3}\right\}$ and $D:=\operatorname{det}\left(\frac{\partial H_{3}}{\partial \theta_{2}}\right)=\mathbf{T}_{2} \overline{\mathbf{R}}_{3}-\overline{\mathbf{T}}_{2} \mathbf{R}_{3}$. Since the derivatives of $h_{1}$ and $h_{2}$ are identically zero, see Eq. (3.13), the required derivatives are

$$
F_{x y}=\left[\begin{array}{ll}
\frac{\partial H_{3}}{\partial \theta_{1}} & \frac{\partial H_{3}}{\partial \theta_{2}} \\
\frac{\partial D}{\partial \theta_{1}} & \frac{\partial D}{\partial \theta_{2}}
\end{array}\right]=\left[\begin{array}{cc}
\frac{\partial H_{3}}{\partial \theta_{1}} & D \\
\frac{\partial D}{\partial \theta_{1}} & \frac{\partial D}{\partial \theta_{2}}
\end{array}\right]
$$

Since $D=0$ at the critical points, only the diagonal elements contribute to $\operatorname{det} F_{x y}$ :

$$
\operatorname{det} F_{x y}=\frac{\partial H_{3}}{\partial \theta_{1}} \cdot \frac{\partial D}{\partial \theta_{2}}
$$

The first factor,

$$
\frac{\partial H_{3}}{\partial \theta_{1}}=i a_{1}\left(T_{1} \overline{\mathbf{R}}_{3}-\mathbf{R}_{3} \overline{\mathbf{T}}_{1}\right)
$$

implies that links 1 and 3 are parallel, and since the turning points happen where links 2 and 3 are parallel, we have critical points wherever the linkage folds flat, all in one line. To check the formulation, we solved the system $\left\{f, D, \frac{\partial H_{3}}{\partial \theta_{1}}\right\}=0$ using polynomial continuation as implemented in the Bertini software package [2]. Since $a_{3}$ only appears as $a_{3}^{2}$, we replaced it by a new variable, say $z=a_{3}^{2}$. Then, with variable groups $\left\{\mathbf{T}_{1}, \mathbf{T}_{2}\right\},\left\{\overline{\mathbf{T}}_{1}, \overline{\mathbf{T}}_{2}\right\},\{z\}$, a three-homogeneous homotopy had six paths. As expected, four of these lead to the solutions:

$$
\begin{aligned}
& a_{3}^{2}=\left(a_{1}+a_{2}+a_{4}\right)^{2} ; \\
& a_{3}^{2}=\left(a_{4}+a_{1}-a_{2}\right)^{2} ; \\
& a_{3}^{2}=\left(a_{4}-a_{1}+a_{2}\right)^{2} ; \\
& a_{3}^{2}=\left(-a_{4}+a_{1}+a_{2}\right)^{2},
\end{aligned}
$$

which are consistent with the four-bar transition linkage formulations of [14]. The remaining two solutions gave $a_{3}=0$ with the two possible ways for the dyad of links 1 and 2 to reach the pivot where link 3 attaches to ground. Using $\mathbf{T}_{2} \overline{\mathbf{T}}_{2}=1$ and Eq. (2.18), the second factor may be written as

$$
\frac{\partial D}{\partial \theta_{2}}=i\left(\mathbf{T}_{2} \overline{\mathbf{R}}_{3}+\overline{\mathbf{T}}_{2} \mathbf{R}_{3}-2 a_{2}\right)
$$


The solution of $\left\{f, D, \frac{\partial H_{3}}{\partial \theta_{2}}\right\}=0$ using Bertini again used six homotopy paths, but in this case, all paths went to infinity. As the example is a general one, this implies that this system is incompatible and will never give critical points for any set of $\left\{a_{1}, a_{2}, a_{4}\right\}$. This is in agreement with existing knowledge about four-bars, where the conditions Eq. (3.25) are known to mark the transitions between mechanisms with different branch numbers.

\subsection{Generate the Singularity Trace}

This problem is analogous to generating the motion curve, except this time we wish to trace out the singularity trace $\mathcal{K}$ instead of one of the motion curves $\mathcal{C}_{p}$. Fortunately, we already have in place all the pieces required. In following the five step process of Section 3.3, the adjustments are:

1. Find the critical points.

2. Sort by parameter value $p$.

3. Solve for turning points at regular test values of $p$ between the critical ones.

4. Track paths of the turning points using the turning point system $F=0$ to the neighboring critical values of $p$.

5. Skip the merge step.

In addition, in Step 3, the branches of the motion curve $\mathcal{C}_{p}$ are found at each of the regular test values of $p$ (Sec. 3.3). This indicates the character of all the mechanisms in the corresponding interval between the critical points. In particular, the number of branches is constant in each interval. 


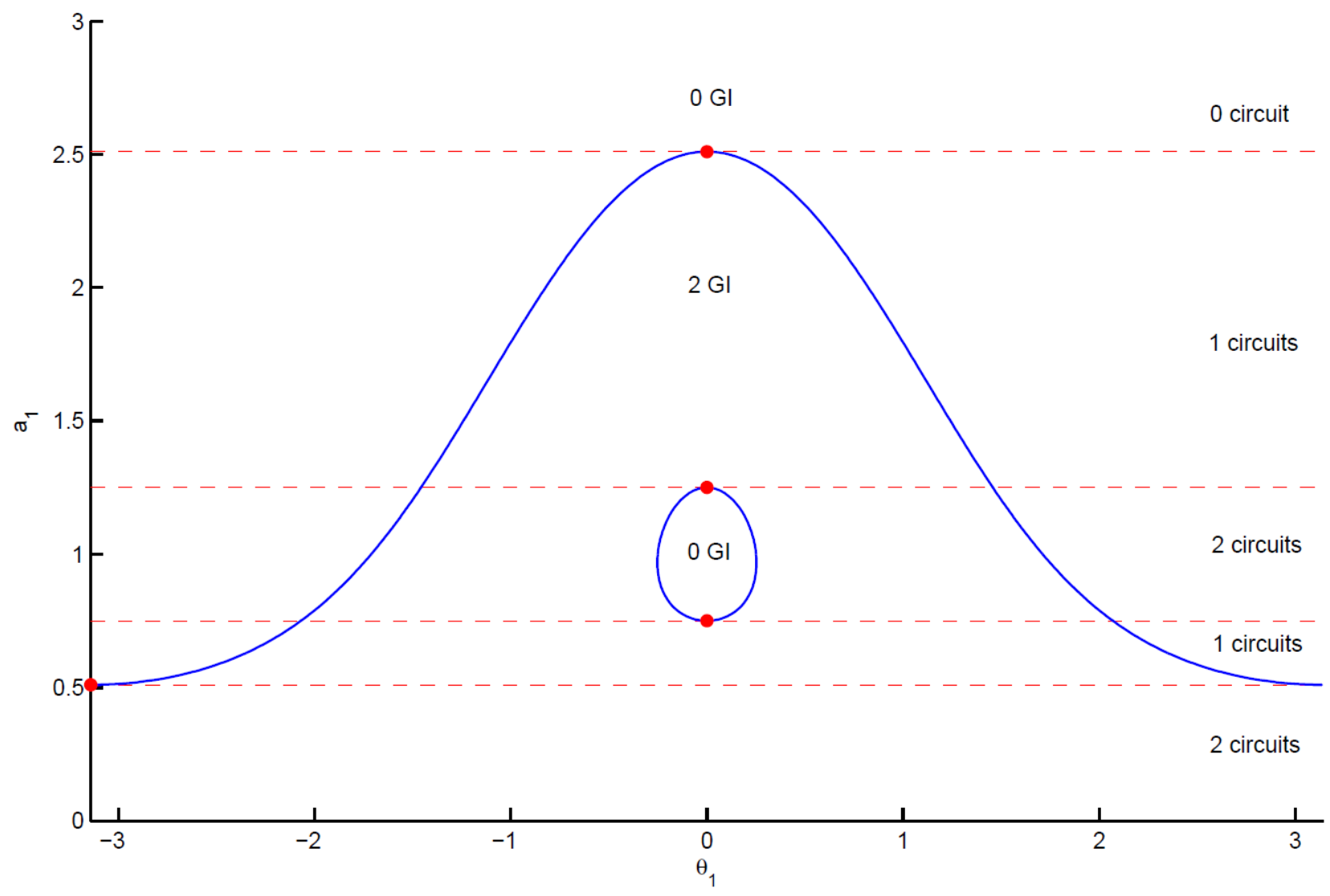

Figure 3.3: Projections of the singularity trace of the four-bar linkage, critical points, shown with red markers, have no $x$ ( $a_{1}$ in this case) component. 


\section{CHAPTER IV}

\section{ANALYSIS EXAMPLES OF TYPICAL LINKAGES}

As discussed in Chapter III, the four-bar linkage analysis was used to show the basic idea and process of the analysis method applied in this thesis. This mechanism, however has just one loop which limits the display of the benefits of this method. This chapter presents the analysis of more complex linkages with multiple loops. The Watt II linkage, a combination of two four-bar mechanisms, is illustrated first to show some common characteristics of the linkages composed of four-bar loop closures. The Stephenson III linkage, analyzed next, is observed to have the property that the input angle is able to rotate more than one revolution between singularities[1]. This motivates a detailed study on several Stephenson III linkages. As a very complex linkage with three five-bar loops, the double butterfly linkage is an excellent test case of the efficiency of this analysis method. To illustrate that this method can be applied to spatial linkages, a spherical four-bar linkage is studied.

\subsection{Watt II Linkage}

The Watt II linkage shown in Fig. 4.1 provides the first example. For the Watt II linkage, the input angle is $x=\theta_{1}$ and the design parameter is $p=a_{1}$. The remaining passive joint variables are $y=\left\{\theta_{2}, \theta_{3}, \theta_{6}, \theta_{7}\right\}$. The physical parameters are $\theta_{4}, \theta_{8}, \alpha_{35}$, and $a_{2}, \ldots, a_{8}$. 


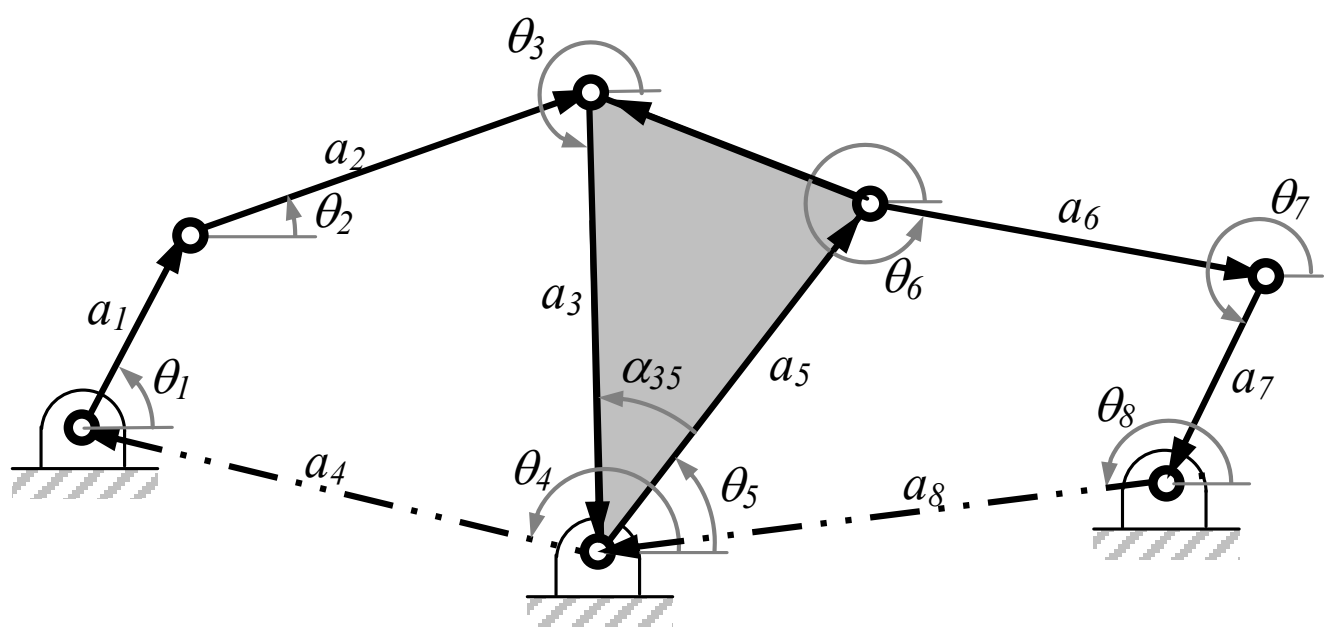

Figure 4.1: Watt II linkage position vector loop.

\subsubsection{Loop Closure}

For the two loops in the Watt II linkage, the isotropic form of the closure condition along with the conjugate identities are

$$
\begin{aligned}
& g_{1}:=a_{1} \mathbf{T}_{1}+a_{2} \mathbf{T}_{2}+a_{3} \mathbf{T}_{3}+a_{4} \mathbf{T}_{4}=0 \\
& \bar{g}_{1}:=a_{1} \overline{\mathbf{T}}_{1}+a_{2} \overline{\mathbf{T}}_{2}+a_{3} \overline{\mathbf{T}}_{3}+a_{4} \overline{\mathbf{T}}_{4}=0 \\
& g_{2}:=b_{5} \mathbf{T}_{3}+a_{6} \mathbf{T}_{6}+a_{7} \mathbf{T}_{7}+a_{8} \mathbf{T}_{8}=0 \\
& \bar{g}_{2}:=b_{5}^{\prime} \overline{\mathbf{T}}_{3}+a_{6} \overline{\mathbf{T}}_{6}+a_{7} \overline{\mathbf{T}}_{7}+a_{8} \overline{\mathbf{T}}_{8}=0 \\
& h_{j}:=\mathbf{T}_{j} \overline{\mathbf{T}}_{j}-1=0, \quad j=2,3,6,7
\end{aligned}
$$

All of the $a_{j}$ are real link lengths, but $b_{5}$ is a complex stretch rotation to properly model the ternary link. That is, $b_{5}=a_{5}\left(\cos \alpha_{35}+i \sin \alpha_{35}\right)$, and $b_{5}^{\prime}$ is the complex conjugate of $b_{5}$. Note that $\mathbf{T}_{4}, \overline{\mathbf{T}}_{4}$ and $\mathbf{T}_{8}, \overline{\mathbf{T}}_{8}$ model the ground link and are known. For a fixed design variable, the system of four equations describes a curve in the four dimensional space of $\left\{\mathbf{T}_{2}, \mathbf{T}_{6}, \overline{\mathbf{T}}_{2}, \overline{\mathbf{T}}_{6}\right\}$. In the numerical 
examples that follow, the values used for the physical parameters are: $a_{2}=4.5, a_{3}=5.0, a_{4}=6.0$, $a_{5}=4.5, a_{6}=2.5, a_{7}=2.0, a_{8}=5.5, \theta_{4}=3.1416, \theta_{8}=3.9270, \alpha_{35}=0.5236$.

\subsubsection{Forward Kinematics}

Since the loop equations, $g_{1}, g_{2}$, and the conjugate loop equations, $\bar{g}_{1}, \bar{g}_{1}$ are all linear, Eq. 4.1 can be rewritten to eliminate variables. To this end, two variables per loop are eliminated by defining,

$$
\begin{aligned}
& \mathbf{R}_{2}:=-a_{2} \mathbf{T}_{2}=a_{1} \mathbf{T}_{1}+a_{3} \mathbf{T}_{3}+a_{4} \mathbf{T}_{4} \\
& \overline{\mathbf{R}}_{2}:=-a_{2} \overline{\mathbf{T}}_{2}=a_{1} \overline{\mathbf{T}}_{1}+a_{3} \overline{\mathbf{T}}_{3}+a_{4} \overline{\mathbf{T}}_{4} \\
& \mathbf{R}_{7}:=-a_{7} \mathbf{T}_{7}=b_{5} \mathbf{T}_{3}+a_{6} \mathbf{T}_{6}+a_{8} \mathbf{T}_{8} \\
& \overline{\mathbf{R}}_{7}:=-a_{7} \overline{\mathbf{T}}_{7}=b_{5}^{\prime} \overline{\mathbf{T}}_{3}+a_{6} \overline{\mathbf{T}}_{6}+a_{8} \overline{\mathbf{T}}_{8}
\end{aligned}
$$

With $a_{i} \neq 0, i=2,7, a_{i}^{2} h_{i}=0$ is formed to achieve

$$
\begin{aligned}
& H_{2}:=\mathbf{R}_{2} \overline{\mathbf{R}}_{2}-a_{2}^{2}=0, \\
& H_{7}:=\mathbf{R}_{7} \overline{\mathbf{R}}_{7}-a_{7}^{2}=0 .
\end{aligned}
$$

The identities corresponding to the remaining joint variables are

$$
\begin{aligned}
& h_{1}:=\mathbf{T}_{1} \overline{\mathbf{T}}_{1}-1=0, \\
& h_{3}:=\mathbf{T}_{3} \overline{\mathbf{T}}_{3}-1=0, \\
& h_{6}:=\mathbf{T}_{6} \overline{\mathbf{T}}_{6}-1=0,
\end{aligned}
$$

For forward kinematics, the input angle $\theta_{1}$ is given, and therefore $\mathbf{T}_{1}$ and $\overline{\mathbf{T}}_{1}$ are known and Eq. 4.5 is not used. The forward kinematics solutions are the roots of Eqs. (4.3),(4.4),(4.6),(4.7), a system of four bilinear equations in the two-homogeneous variable groups $\left\{\mathbf{T}_{3}, \mathbf{T}_{6}\right\},\left\{\overline{\mathbf{T}}_{3}, \overline{\mathbf{T}}_{6}\right\}$. Solution of the polynomial systems can be readily accomplished by numerical polynomial continuation [4] using the Bertini software package [2]. Real solutions are those for which $\left|\mathbf{T}_{3}\right|=\left|\mathbf{T}_{6}\right|=1$. 
At any point on the curve, the remaining angles can be solved by using

$$
\mathbf{T}_{2}=-\mathbf{R}_{2} / a_{2}, \quad \mathbf{T}_{7}=-\mathbf{R}_{7} / a_{7}
$$

\subsubsection{Singularity Points}

The singularity points are given by a system of equations consisting of the five loop closure conditions Eqs. (4.3)-(4.7) along with the additional condition

$$
D:=\operatorname{det}\left[\begin{array}{ll}
\frac{\partial H_{2}}{\partial \theta_{3}} & \frac{\partial H_{2}}{\partial \theta_{6}} \\
\frac{\partial H_{7}}{\partial \theta_{3}} & \frac{\partial H_{7}}{\partial \theta_{6}}
\end{array}\right]=0 .
$$

It is noted that $\frac{\partial H_{2}}{\partial \theta_{6}}=0$ since $\mathbf{T}_{6}$ does not appear in $\mathbf{R}_{2}$. This system of six equations in six unknowns is separated into two-homogeneous variable groups, $\left\{\mathbf{T}_{1}, \mathbf{T}_{2}, \mathbf{T}_{6}\right\}$ and $\left\{\overline{\mathbf{T}}_{1}, \overline{\mathbf{T}}_{2}, \overline{\mathbf{T}}_{6}\right\}$. Solving the system using Bertini [2], at most 8 turning points are found in a general example. When $a_{1}=2.0,2$ of 16 singularity points are real. When $a_{1}=6.0$ and $a_{1}=12.0,4$ of 16 singularity points are real. When $a_{1}=9.0,6$ of 16 singularity points are real.

\subsubsection{Motion Curve}

A trace of a motion curve with $a_{1}=2.0$ is projected onto the $\theta_{1}-\theta_{6}$ plane and shown in Fig. 4.2. Note that the linkage has one circuit and two singularity points at $\theta_{1}=-0.2061,2.0652$. Zero GIs exist for $\theta_{1}<-0.2061$ and $\theta_{1}>2.0652$. Two GIs exist for $-0.2061<\theta_{1}<2.0652$.

A second trace of a motion curve with $a_{1}=9.0$ is projected onto the $\theta_{1}-\theta_{6}$ plane and shown in Fig. 4.3. Note the motion curve has two circuits and six of singularity points at $\theta_{1}=-0.2427$, $0.6019,0.6314,1.2277,1.3205,1.3205$. Notice that two signulatiries occur at the same value of $\theta_{1}$. This is associated with singularities within the first four-bar loop, for which two inversions of the second four-bar loop exist. The linkage has zero GIs for $\theta_{1}<-0.2427,0.6019<\theta_{1}<0.6314$ and $\theta_{1}>1.3205$. Two GIs exist for $-0.2427<\theta_{1}<0.6019$ and $0.6314<\theta_{1}<1.2277$. Four GIs exist for all values of $1.2277<\theta_{1}<1.3205$. 


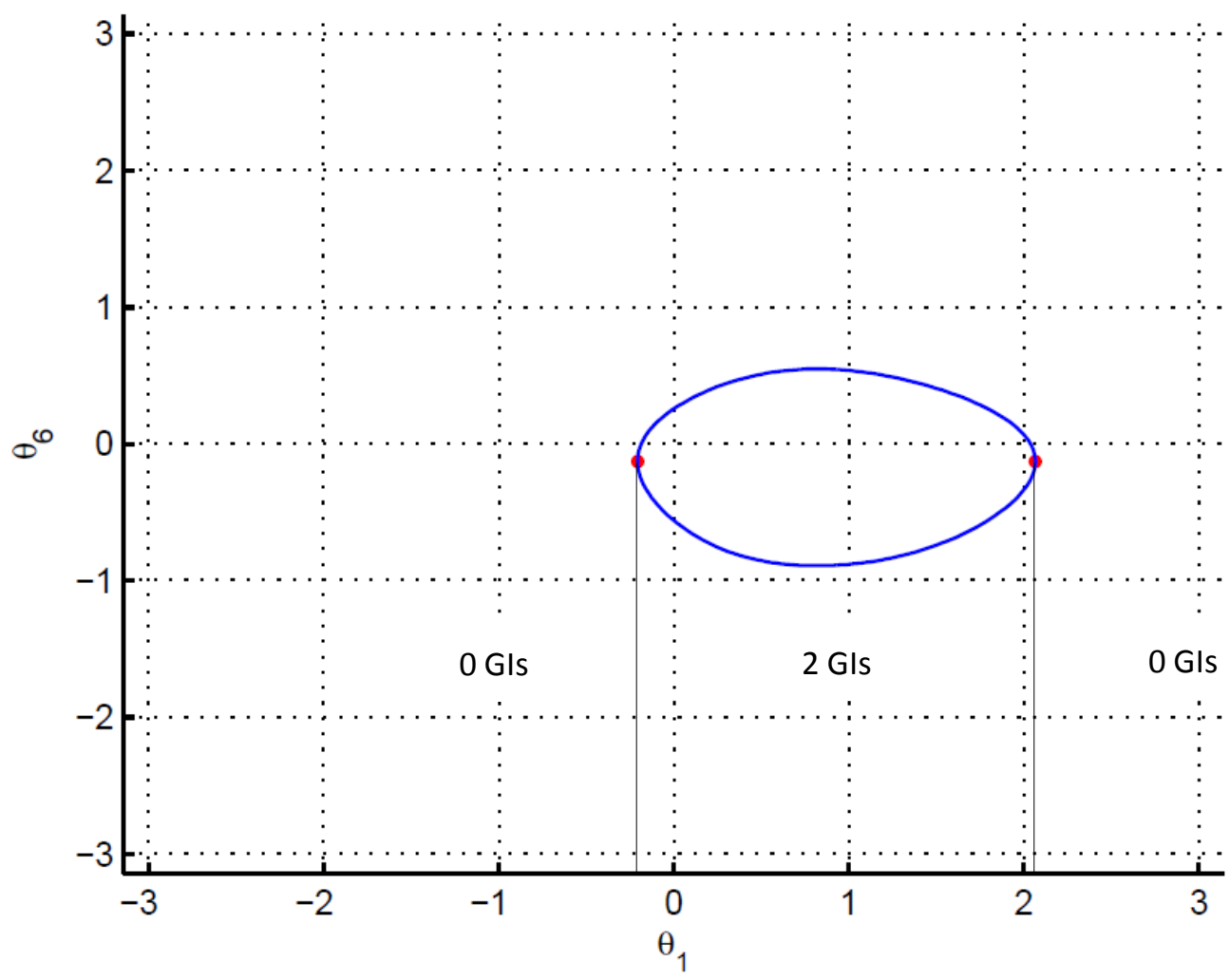

Figure 4.2: Trace of the motion curve for the Watt II linkage, with $a_{1}=2.0$, projected onto the $\theta_{1}-\theta_{6}$ plane.

\subsubsection{Critical Points}

The process of determining the critical points proceeds analogously to finding singularity points, replacing $x$ by $p, y$ by $(x, y)$, and $f$ by $(f, D)$. The critical points occur when $(\Delta x, \Delta y) \neq 0$ with $\Delta p=0$, which implies,

$$
E:=\operatorname{det}\left[\begin{array}{ll}
\frac{\partial f}{\partial x} & \frac{\partial f}{\partial y} \\
\frac{\partial D}{\partial x} & \frac{\partial D}{\partial y}
\end{array}\right]=0
$$




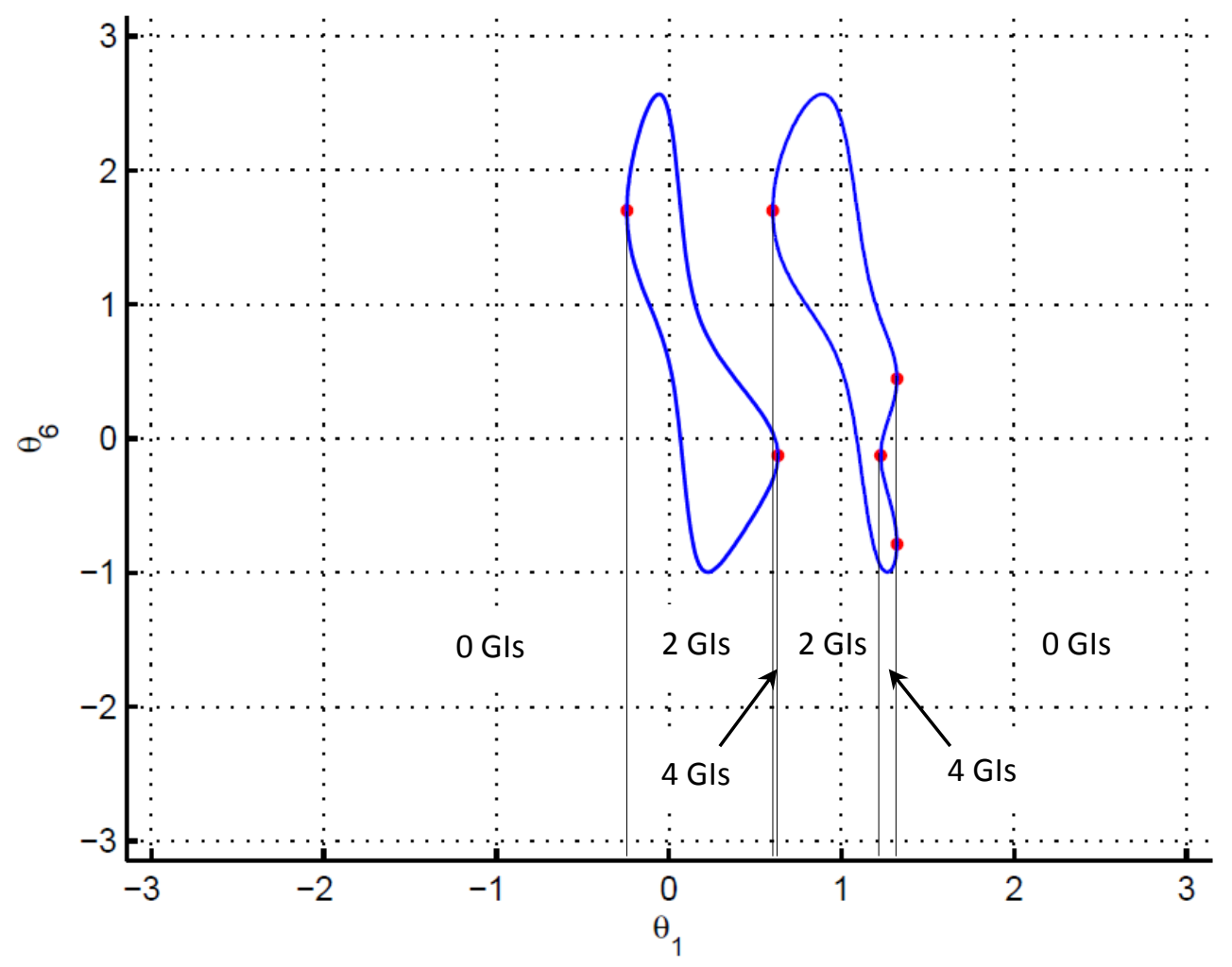

Figure 4.3: Trace of the motion curve for the Watt II linkage, with $a_{1}=9.0$, projected onto the $\theta_{1}-\theta_{6}$ plane.

Considering $a_{1}$ as a variable, the critical points for the Watt II are determined by the solution of Eqs. (4.5)-(4.4),(4.9) along with,

$$
E:=\operatorname{det}\left[\begin{array}{lll}
\frac{\partial H_{2}}{\partial \theta_{1}} & \frac{\partial H_{2}}{\partial \theta_{3}} & \frac{\partial H_{2}}{\partial \theta_{6}} \\
\frac{\partial H_{7}}{\partial \theta_{1}} & \frac{\partial H_{7}}{\partial \theta_{3}} & \frac{\partial H_{7}}{\partial \theta_{6}} \\
\frac{\partial D}{\partial \theta_{1}} & \frac{\partial D}{\partial \theta_{3}} & \frac{\partial D}{\partial \theta_{6}}
\end{array}\right]=0 .
$$

It is noted that $\frac{\partial H_{2}}{\partial \theta_{6}}=0$, since $\mathbf{T}_{6}$ does not appear in $\mathbf{R}_{2}$, and $\frac{\partial H_{7}}{\partial \theta_{1}}=0$, since $\mathbf{T}_{1}$ does not appear in $\mathbf{R}_{7}$. This forms a system of seven equations in the seven unknowns $a_{1},\left\{\mathbf{T}_{1}, \mathbf{T}_{2}, \mathbf{T}_{6}\right\},\left\{\overline{\mathbf{T}}_{1}, \overline{\mathbf{T}}_{2}, \overline{\mathbf{T}}_{6}\right\}$. Using Bertini to solve, critical points appear in pairs, existing at $a_{1}=0.4614,5.7107,6.2872$, $6.4642,7.5578,9.4614,15.2132,15.2872$. 


\subsubsection{Singularity Trace}

The singularity trace is shown in Fig. 4.5. The critical points signify local extrema of the singularity curve. Critical points that appear on the singularity trace as smooth extrema represent transition linkages, where there is a change in the number of circuits.

Sampling the motion curve between critical points determines the number of circuits. As identified in [15], the singularity trace separates the plot into zones having the same number of GIs. Solving the forward kinematic problem for one sample point within each zone determines the number in GIs in the zone, as indicated in Fig. 4.5. It commonly occurs that the number of GIs in adjacent regions differ by two.

\subsubsection{Symmetric Property of Singularity Trace}

The singularity trace of a four-bar is symmetric due to the single four-bar loop property. See Sec. 4.3.6. The Watt II linkage is a combination of two four-bar mechanisms connected by a common triangular link. Using the same parameter definitions of Watt II linkage above, if $\alpha_{35}+\theta_{8}=\pi$, then the singularity trace of the Watt II linkage will be symmetric as well.

To illustration this special property, the following physical parameter values are used: $a_{2}=4.0$, $a_{3}=3.5, a_{4}=5.0, a_{5}=3.0, a_{6}=3.8, a_{7}=4.0, a_{8}=5.5, \theta_{4}=\pi, \theta_{8}=2.0944, \alpha_{35}=2.6180$. The singularity trace is shown in Fig. 4.5. The critical points are identified represent transition linkages (where there is a change in the number of circuits). The singularity trace is symmetric and all critical points appear in pairs.

For Watt II linkage with the physical parameters above, the motion curves in each design parameter value are identical but in different orientations, as shown in Fig. 4.6 and Fig. 4.7. With 


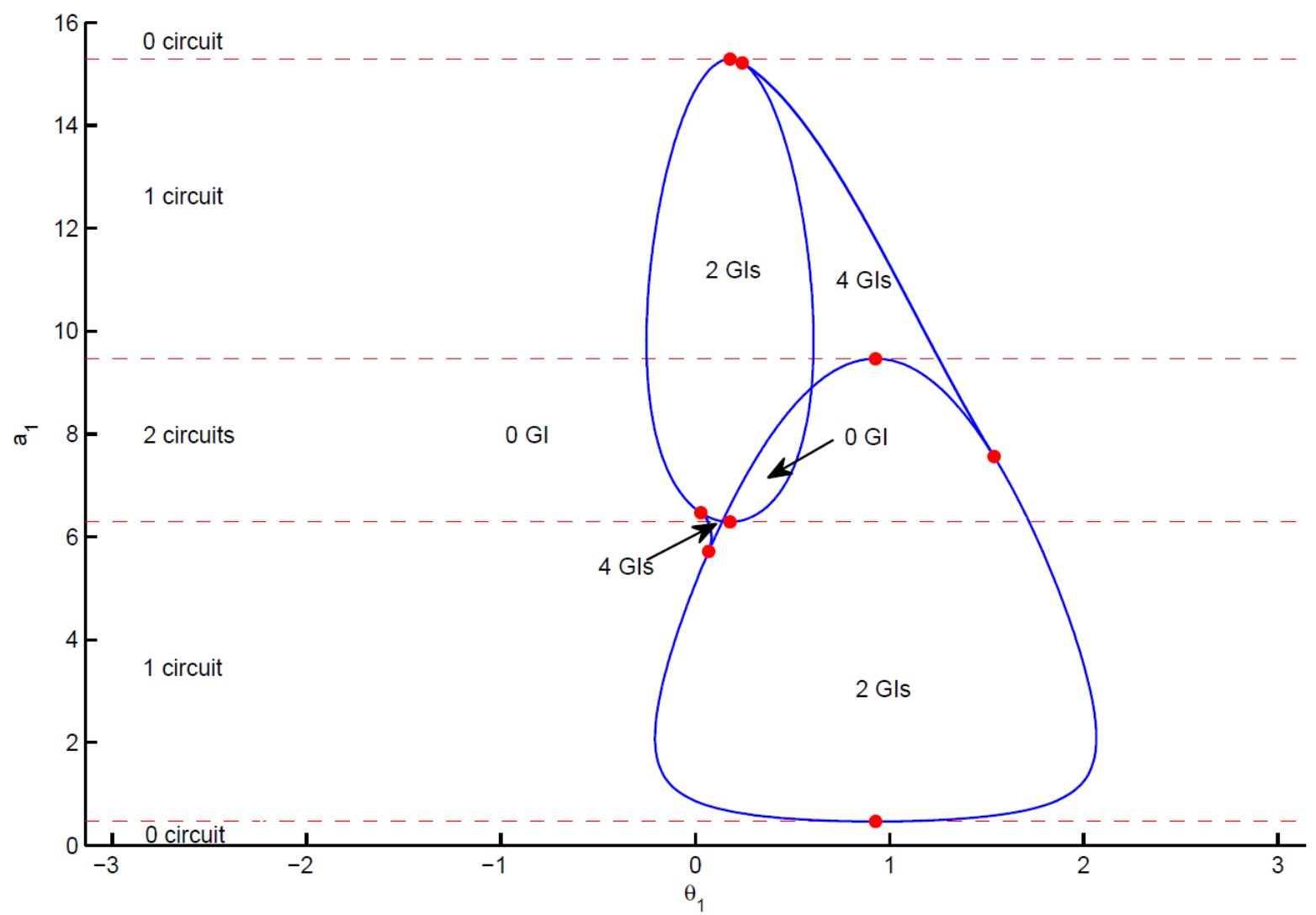

Figure 4.4: Projection of the Watt II singularity trace. Red circles mark the critical points. Regions of equal GIs and circuits are identified.

symmetry in the Watt II motion curves, some of the arcs observed on the singularity trace are actually two coincident arcs. In this case, the number of GIs in adjacent regions change by four. Additionally, with double critical points, the number of circuits changes by two, or does not change.

A trace of a motion curve with $a_{1}=3.5$ is projected onto the $\theta_{1}-\theta_{6}$ plane and shown in Fig. 4.6. Note that the linkage has two circuits and four singularity points at $\theta_{1}=-1.9331,-0.3859,0.3859$, 


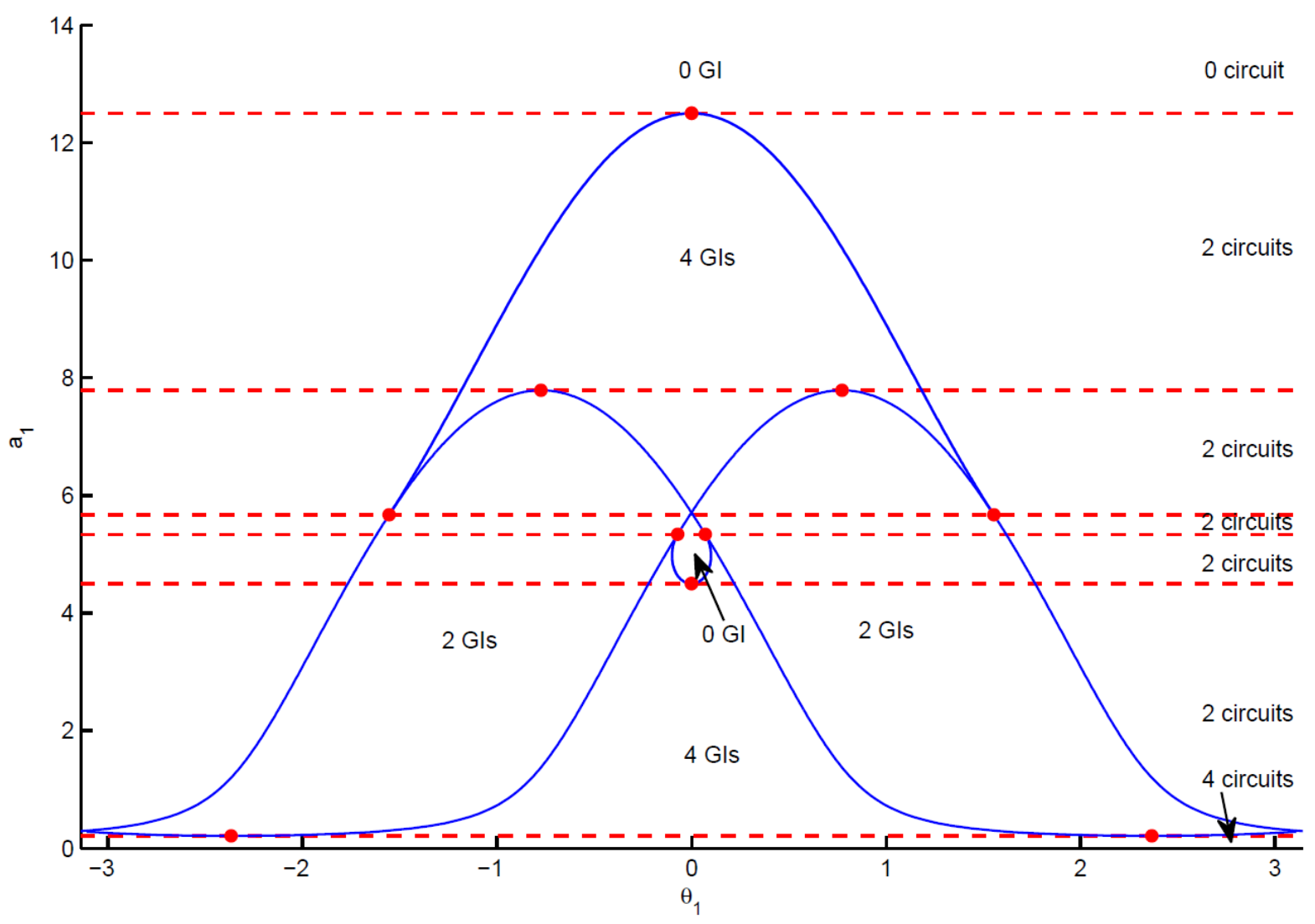

Figure 4.5: Projection of the Watt II singularity trace. Red circles mark the critical points. Regions of equal GIs and circuits are identified.

1.9331. Additionally, 0 GIs exist for $\theta_{1}<-1.9331$ and $\theta_{1}>1.9331,2$ GIs exist for $-1.9331<$ $\theta_{1}<-0.3859$ and $0.3859<\theta_{1}<1.9331$, and 4 GIs exist for $-0.3859<\theta_{1}<0.3859$.

A second trace of a motion curve with $a_{1}=10.0$ is projected onto the $\theta_{1}-\theta_{6}$ plane and shown in Fig. 4.7. Note the symmetry of the curve, exhibiting two circuits and two sets of singularity points at $\theta_{1}=-0.8128,-0.8128,0.8128,0.8128$. The linkage has 0 GIs for $\theta_{1}<-0.8128$ and $\theta_{1}>0.8128,4$ GIs exist for all values of $-0.8128<\theta_{1}<0.8128$. 


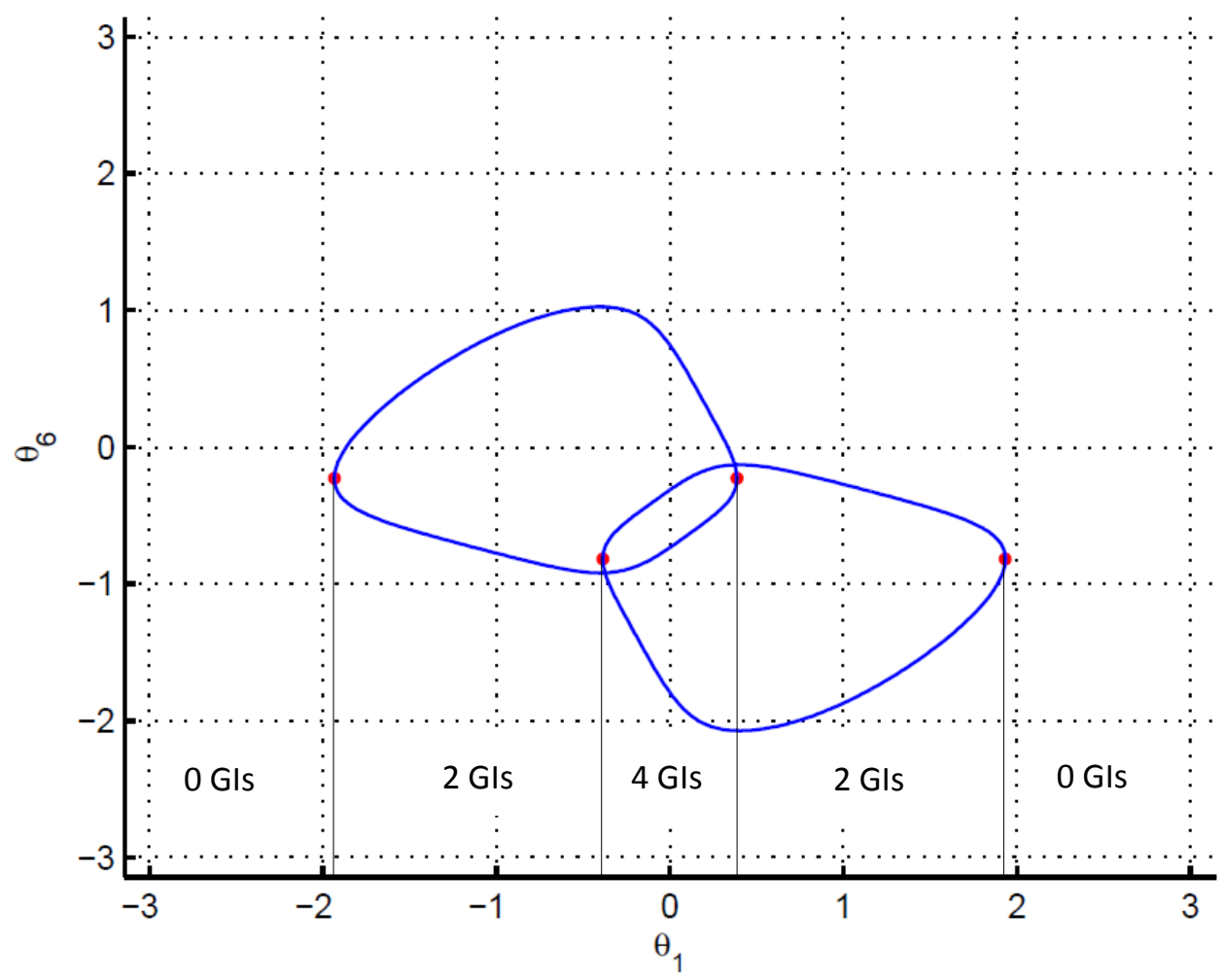

Figure 4.6: Watt II linkage with $a_{1}=3.5$, motion curve trace on $\theta_{1}-\theta_{6}$ plane.

The singularity trace with $a_{1}<0.2117$ has four circuits, with no singularities and four GIs for all $\theta_{1}$. That is, the linkage can be assembled into four different configurations all having a fully rotating input link.

\subsection{Stephenson III Linkage}

For the Stephenson III shown in Fig. 4.8, $x=\theta_{1}$ is considered the input angle and the design parameter is designated as $p=a_{1}$. The remaining joint variables are $y=\left\{\theta_{2}, \theta_{3}, \theta_{4}, \theta_{7}\right\}$. The physical parameters are $\theta_{5}, \theta_{6}, \alpha_{38}$, and $a_{2}, \ldots, a_{8}$. In the numerical examples that follow, the values used for the physical parameters are: $a_{2}=4.0, a_{3}=3.5, a_{4}=4.1, a_{5}=8.0, a_{6}=8.0$, 


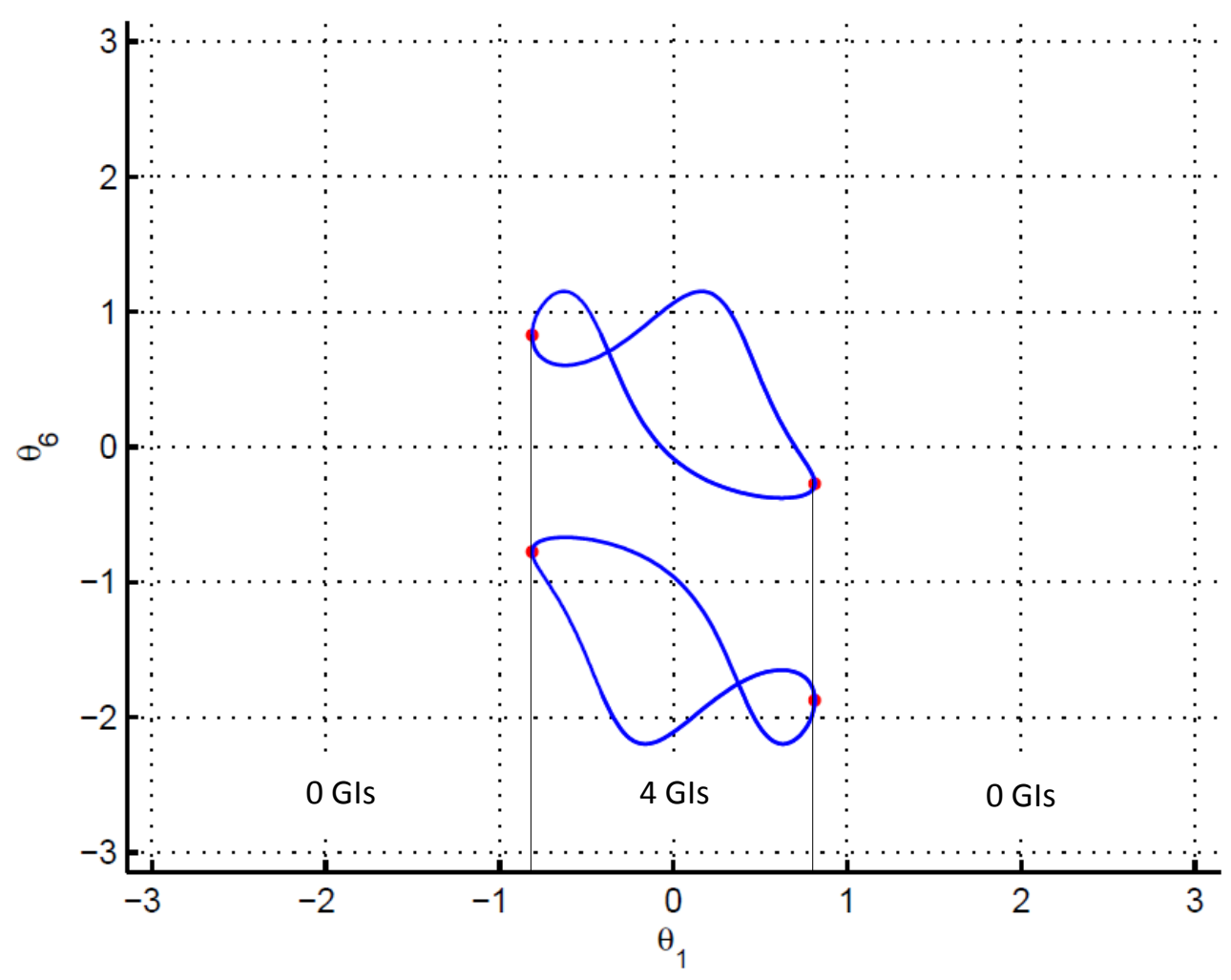

Figure 4.7: Watt II linkage with $a_{1}=10.0$, motion curve trace on $\theta_{1}-\theta_{6}$ plane.

$a_{7}=8.0, a_{8}=5.0, \theta_{5}=1.5708, \theta_{6}=5.8832, \alpha_{38}=0.7752, b_{3}=a_{3} \mathbf{T}_{\alpha_{38}}$ and $b_{3}^{\prime}=a_{3} \overline{\mathbf{T}}_{\alpha_{38}}$. The governing loop, singularity and critical point equations were developed in [1], so only the resulting equations are presented here. The loop closure equations are

$$
\begin{aligned}
& \mathbf{R}_{2}:=a_{2} \mathbf{T}_{2}=-\left(a_{1} \mathbf{T}_{1}+a_{3} \mathbf{T}_{3}+a_{4} \mathbf{T}_{4}+a_{5} \mathbf{T}_{5}\right), \\
& \overline{\mathbf{R}}_{2}:=a_{2} \overline{\mathbf{T}}_{2}=-\left(a_{1} \overline{\mathbf{T}}_{1}+a_{3} \overline{\mathbf{T}}_{3}+a_{4} \overline{\mathbf{T}}_{4}+a_{5} \overline{\mathbf{T}}_{5}\right), \\
& \mathbf{R}_{7}:=a_{7} \overline{\mathbf{T}}_{7}=-\left(b_{3} \mathbf{T}_{3}+a_{4} \mathbf{T}_{4}+a_{6} \mathbf{T}_{6}\right), \\
& \overline{\mathbf{R}}_{7}:=a_{7} \overline{\mathbf{T}}_{7}=-\left(b_{3}^{\prime} \overline{\mathbf{T}}_{3}+a_{4} \overline{\mathbf{T}}_{4}+a_{6} \overline{\mathbf{T}}_{6}\right),
\end{aligned}
$$


Using Eq. (4.12), the closure conditions become

$$
\begin{aligned}
h_{1} & :=\mathbf{T}_{1} \overline{\mathbf{T}}_{1}-1=0, \\
H_{2} & :=\mathbf{R}_{2} \overline{\mathbf{R}}_{2}-a_{2}^{2}=0, \\
h_{3} & :=\mathbf{T}_{3} \overline{\mathbf{T}}_{3}-1=0, \\
h_{4} & :=\mathbf{T}_{4} \overline{\mathbf{T}}_{4}-1=0, \\
H_{7} & :=\mathbf{R}_{7} \overline{\mathbf{R}}_{7}-a_{7}^{2}=0 .
\end{aligned}
$$

The singularity condition is

$$
D:=\operatorname{det}\left[\begin{array}{ll}
\frac{\partial H_{2}}{\partial \theta_{3}} & \frac{\partial H_{2}}{\partial \theta_{4}} \\
\frac{\partial H_{7}}{\partial \theta_{3}} & \frac{\partial H_{7}}{\partial \theta_{4}}
\end{array}\right]=0 .
$$

Lastly, the condition for critical points is

$$
E:=\operatorname{det}\left[\begin{array}{ccc}
\frac{\partial H_{2}}{\partial \theta_{1}} & \frac{\partial H_{2}}{\partial \theta_{3}} & \frac{\partial H_{2}}{\partial \theta_{4}} \\
0 & \frac{\partial H_{7}}{\partial \theta_{3}} & \frac{\partial H_{7}}{\partial \theta_{4}} \\
\frac{\partial D}{\partial \theta_{1}} & \frac{\partial D}{\partial \theta_{3}} & \frac{\partial D}{\partial \theta_{4}}
\end{array}\right]=0 .
$$

\subsubsection{Critical Points}

The system of equations for determination of critical points consists of Eqs. (4.13)-(4.17),(4.18),(4.19). Considering $a_{1}$ as a variable design paramater, this system of seven equations in the seven unknowns

$a_{1},\left\{\mathbf{T}_{1}, \mathbf{T}_{3}, \mathbf{T}_{4}\right\},\left\{\overline{\mathbf{T}}_{1}, \overline{\mathbf{T}}_{3}, \overline{\mathbf{T}}_{4}\right\}$. Using Bertini to solve, critical points exist at $a_{1}=1.1114,2.0471$, 2.0551, 5.9129, 5.9529, 5.9728, 7.4384, 7.5190, 8.7611, 8.8284, 10.0551, 10.9436, 13.7642, 13.9129, 17.4641, 18.9436 .

\subsubsection{Singularity Trace}

The singularity trace with $a_{1}$ as a variable is shown in Fig. 4.9. Unlike the Watt II linkage, some critical points appear as cusps on the singularity trace. Solving the forward kinematic problem for one sample point within each region bounded by the singularity curve determines the number of GIs 


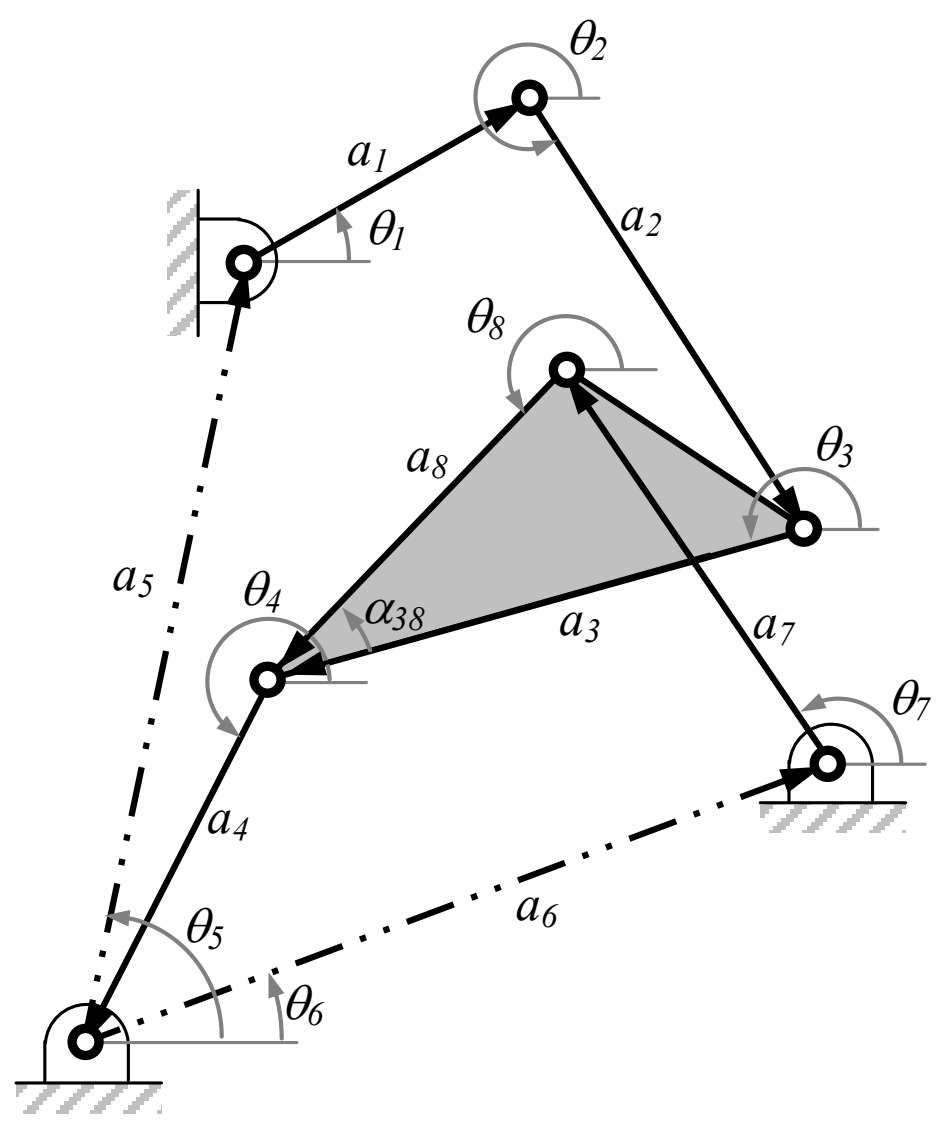

Figure 4.8: Stephenson III linkage position vector loop.

in that entire region. The number of GIs are noted in Fig. 4.9. Additionally, the number of circuits changes by one at each non-cusp extrema and are also noted in Fig. 4.9.

\subsubsection{Motion Curves}

A trace of the motion curve with $a_{1}=1.1$ is projected onto the $\theta_{1}-\theta_{3}$ plane and shown in Fig. 4.10. This value of $a_{1}$ is slightly less than the smallest critial point value (1.1114). Note that this lowest critical point appears as a cusp in Fig. 4.9. This linkage has two GIs for all values of 


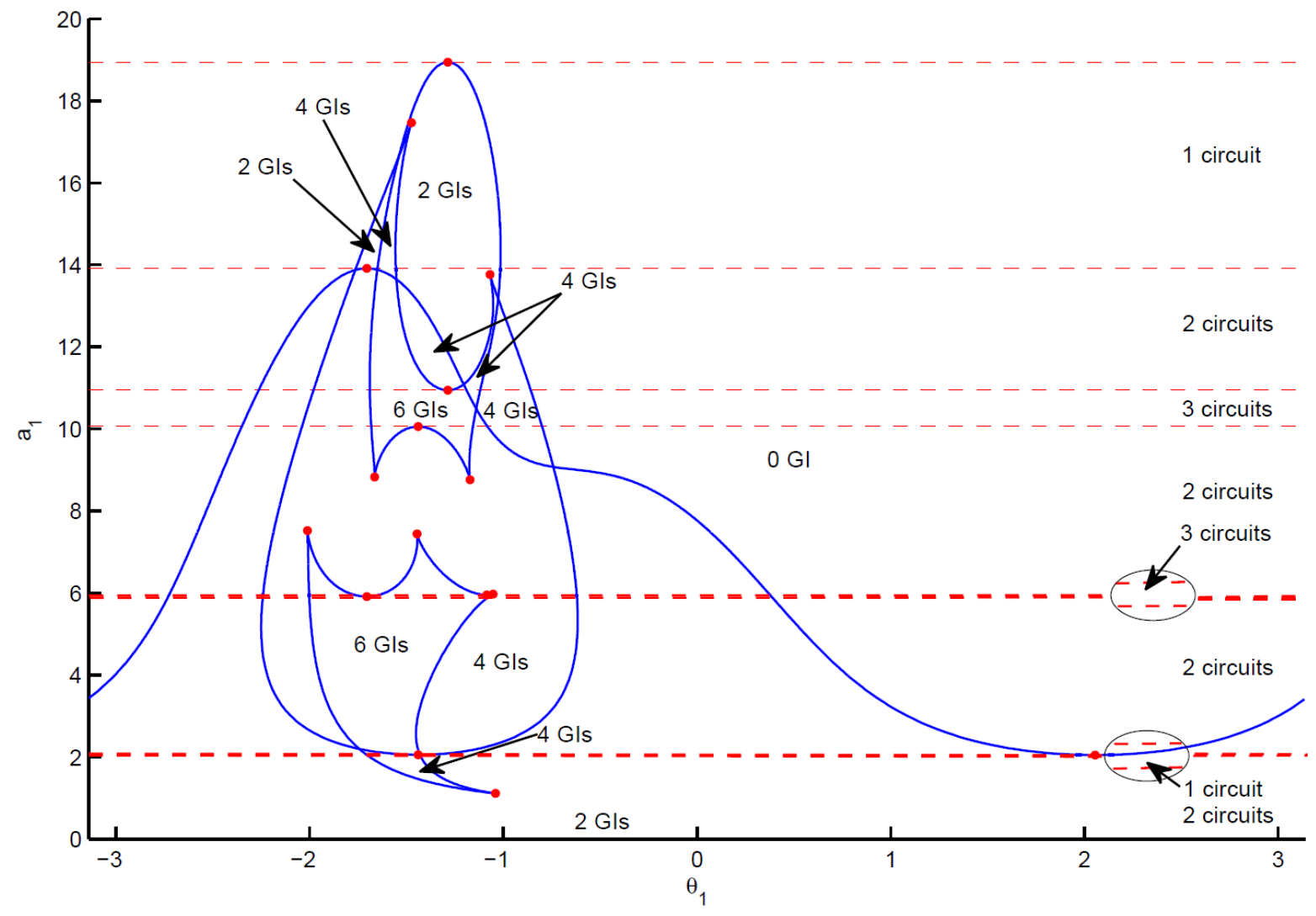

Figure 4.9: Projection of the Stephenson III singularity trace with respect to $a_{1}$. Red circles mark the critical points. Regions of equal GIs and circuits are identified.

$\theta_{1}$, two separate circuits and no singularities. Thus, two linkage configurations have continuously rotating cranks with $a_{1}<1.1114$.

A second trace of the motion curve with $a_{1}=2.0$ is projected onto the $\theta_{1}-\theta_{3}$ plane and shown in Fig. 4.11. This value of $a_{1}$ is slightly greater than the smallest critial point value (1.1114). This linkage has two GIs for $\theta_{1}<-1.6902$ and $\theta_{1}>-1.4259$, four GIs for $-1.6902<\theta_{1}<-1.4259$, two separate circuits and two singularities. The change of $a_{1}$ from 1.1 to 2.0 crosses a cusp in 


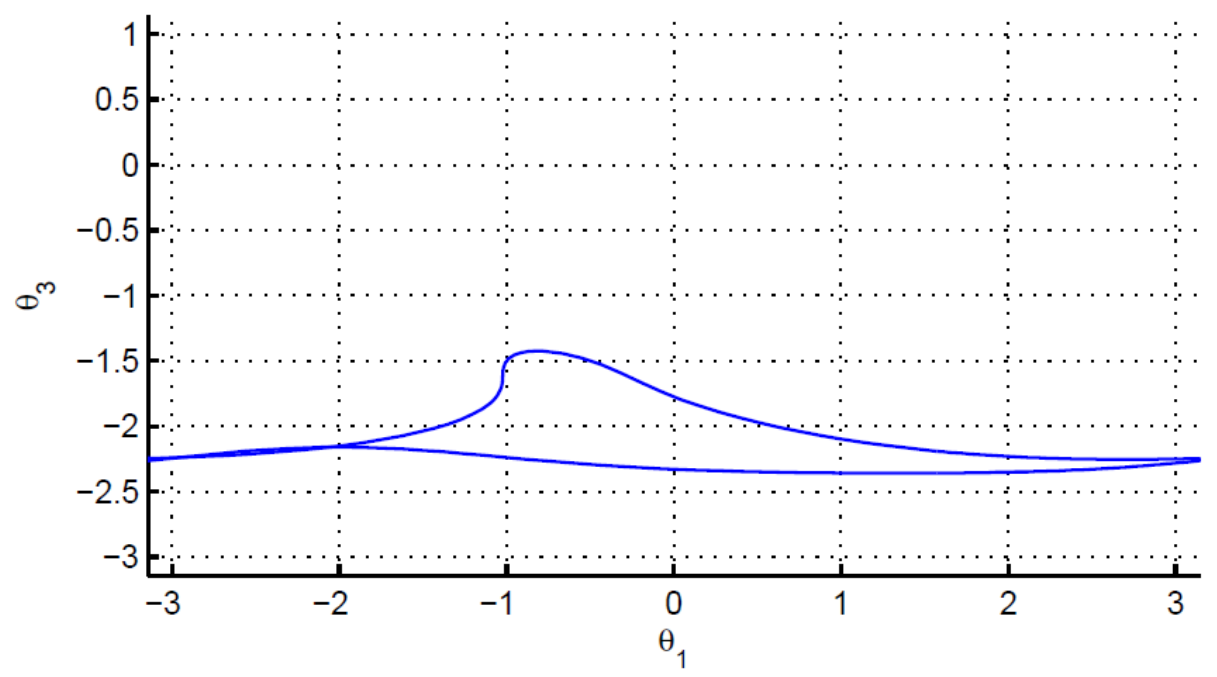

Figure 4.10: Stephenson III linkage motion curve with respect to $\theta_{1}, \theta_{3}$ at $a_{1}=1.1$.

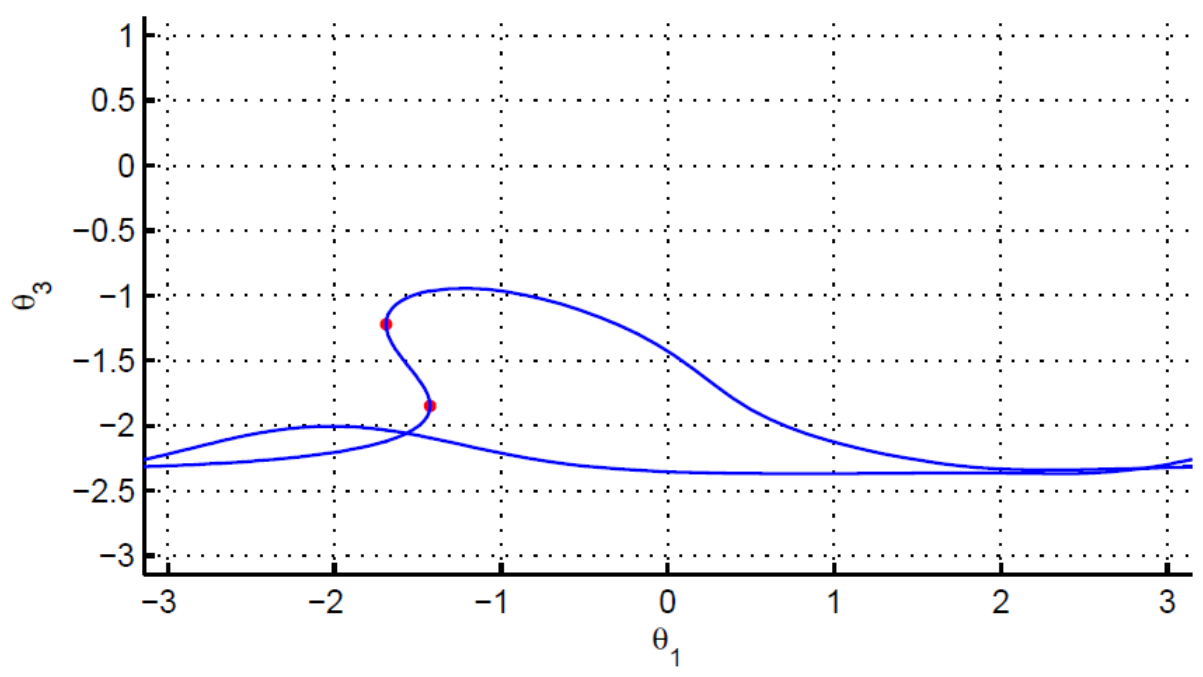

Figure 4.11: Stephenson III linkage motion curve with respect to $\theta_{1}, \theta_{3}$ at $a_{1}=2.0$. 


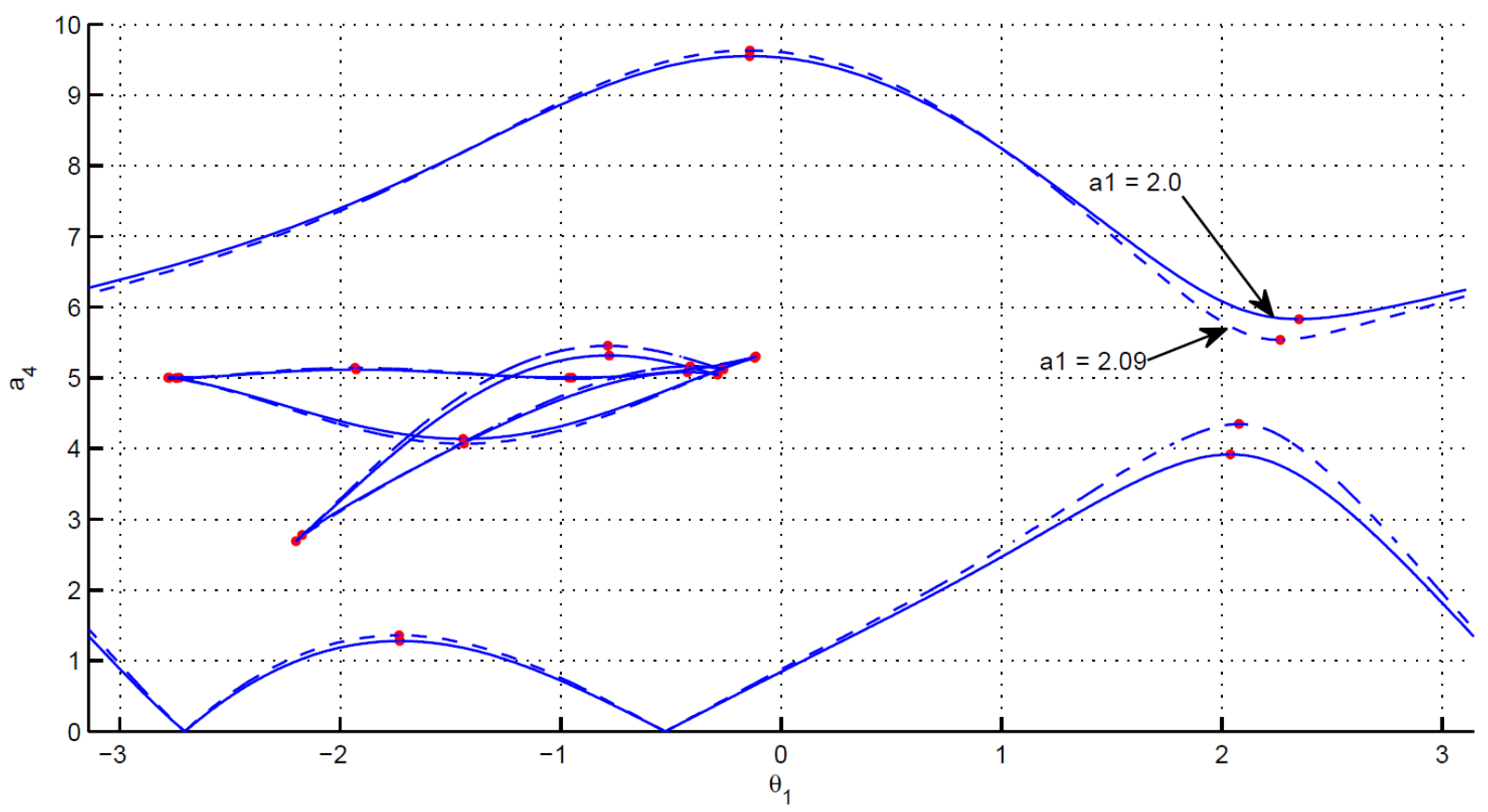

Figure 4.12: Stephenson III singularity trace with respect to $a_{4}$.

Fig. 4.9 and two singularities are introduced without an additional circuit. Thus, the motion curve bends over itself as seen in Fig. 4.11. This results in a linkage that is able to rotate greater than one full revolution between singularities. As observed in [1] and witnessed again here, this net-zero, singularity-free actuation that places the linkage into a different GI is associated with cusps on the singularity trace.

The following analysis considers changes in two design parameters. The motion curve shown in Fig. 4.11 was generated with $a_{1}=2.00$ and $a_{4}=4.10$ and exhibited two circuits, one having a fully rotating crank and the other containing a greater than $360^{\circ}$ branch. Figure 4.12 shows a pair of singulatiry traces with $a_{4}$ considered variable. The solid trace is with $a_{1}=2.00$ and the dashed trace is with $a_{1}=2.09$. Adjusting $a_{1}$ from 2.00 to 2.09 crosses a critical point in Fig. 4.9. 


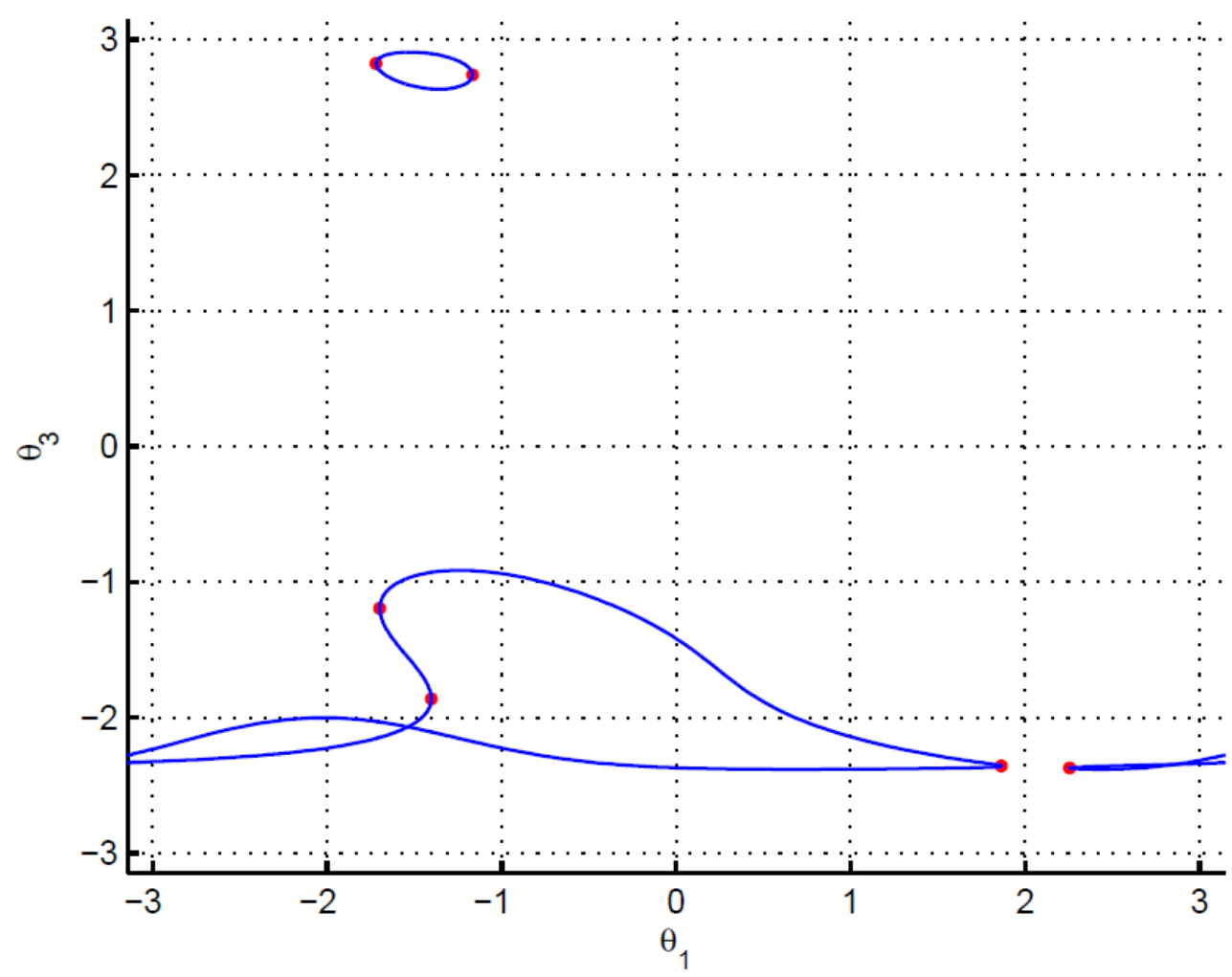

Figure 4.13: Stephenson III motion curve with respect to $a_{1}=2.09, a_{4}=4.15$.

Additionally, adjusting $a_{4}$ from 4.10 to 4.15 crosses the critical point on both traces in Fig. 4.12. The motion curve shown in Fig. 4.13 was generated with $a_{1}=2.09$ and $a_{4}=4.15$. After making both subtle adjustments, the two circuits in Fig. 4.11 have been merged and no GI is filly able to rotate. Moreover, an additional circuit with two branches has been formed. Thus, subtle adjustments to two link lengths, each crossing a corresponding critical point, results in drastically different motion characterisitcs. 


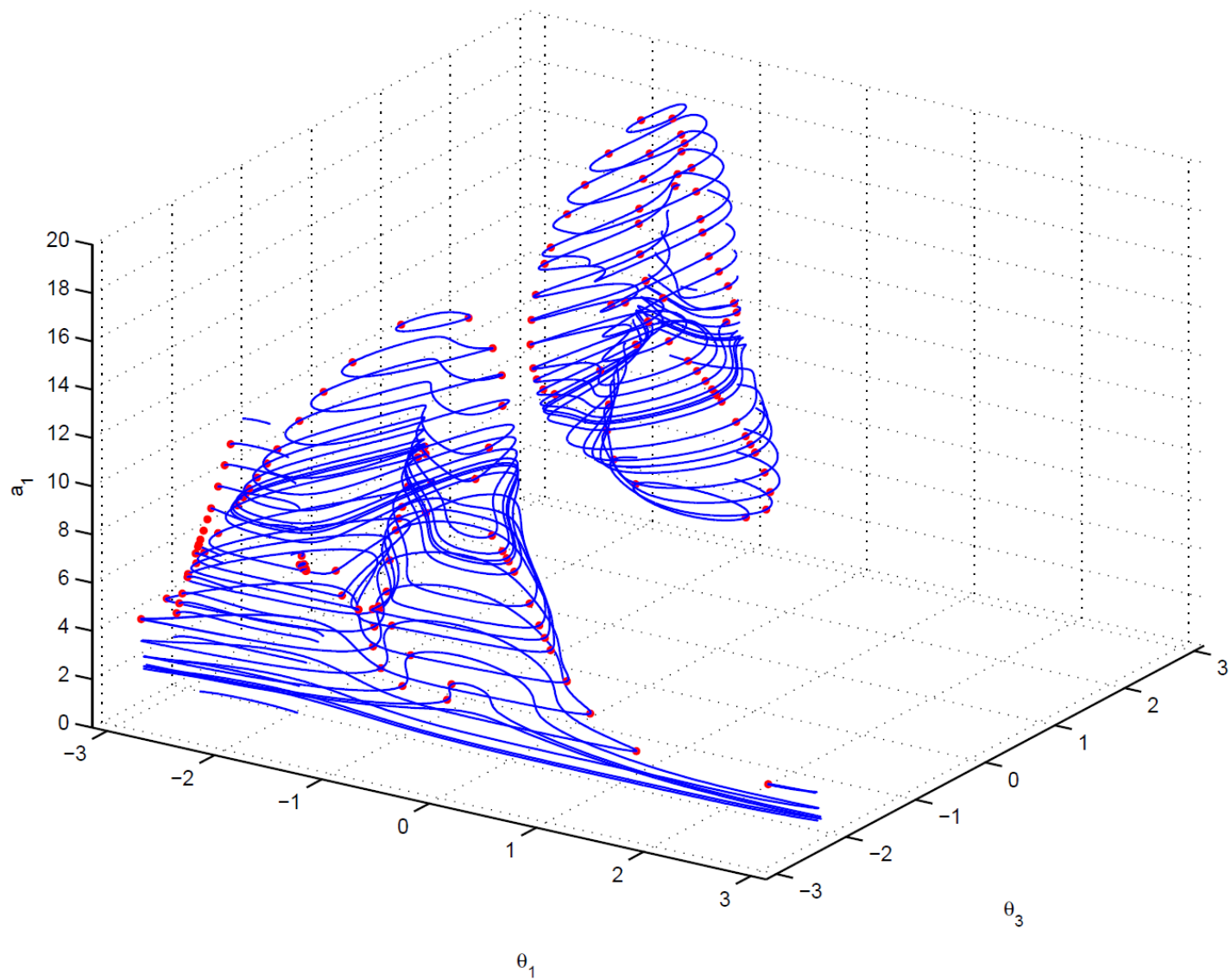

Figure 4.14: Stephenson III design surface is created by stacking motion curves with respect to $\theta_{1}$ and $\theta_{3}$ for increasing values of $a_{1}$.

\subsubsection{Design Surface}

A design surface shows every motion that can be obtained by varying the design parameter. For the Stephenson III linkage with the values given in Sec. 4.2, the design surface is shown in Fig. 4.14 with respect to the design parameter $a_{1}$. 


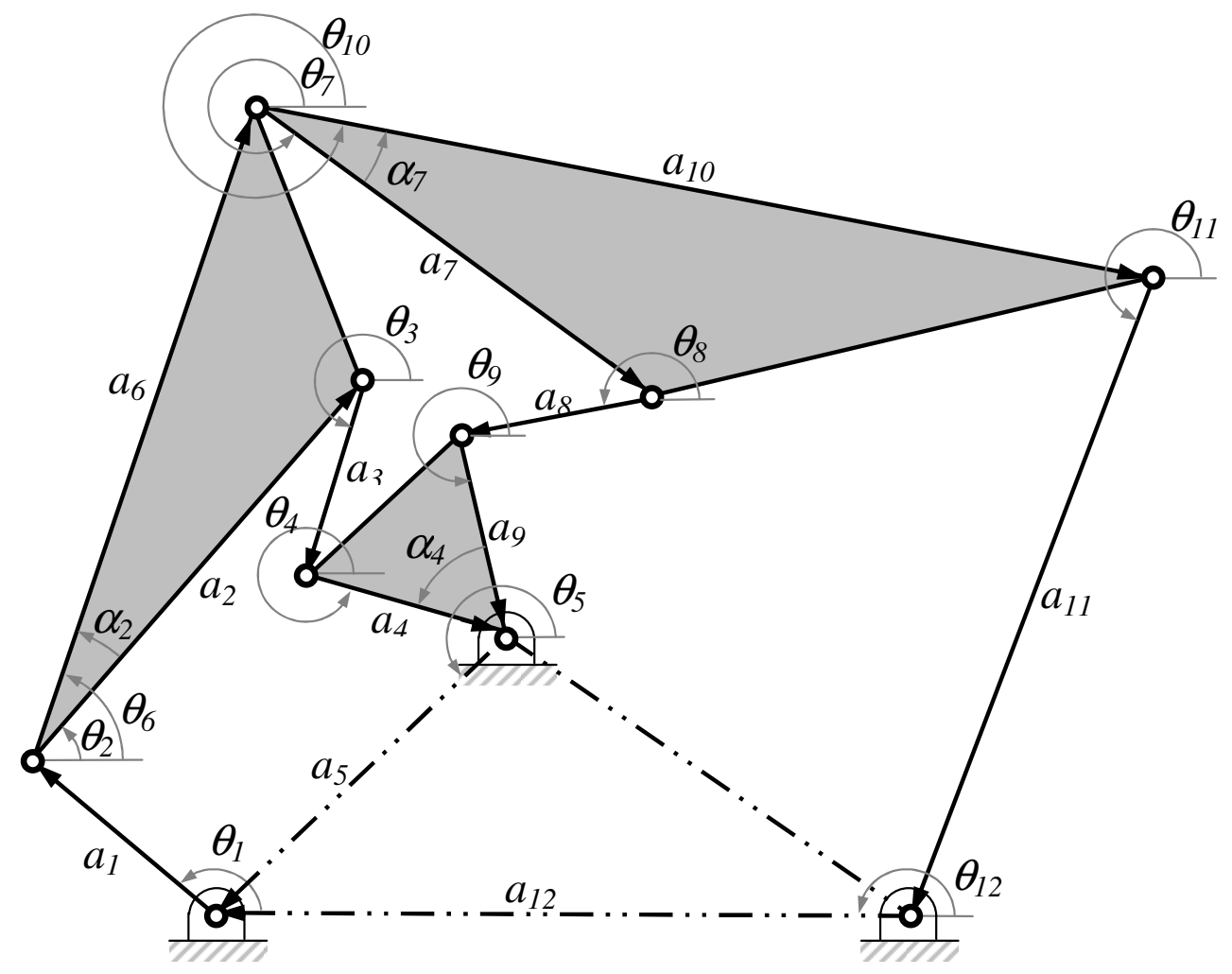

Figure 4.15: Double butterfly linkage position vector loop.

\subsection{Double Butterfly Linkage}

This section applies the general analysis methodology to the double butterfly linkage, as shown in Fig. 4.15. For this case, the input angle is $x=\theta_{1}$ and the design parameter is designated as $p=a_{1}$. The remaining joint variables are $y=\left\{\theta_{2}, \theta_{3}, \theta_{4}, \theta_{7}, \theta_{8}, \theta_{11}\right\}$. The physical parameters are $\theta_{1}, \theta_{5}, \theta_{12}, \alpha_{2}, \alpha_{4}, \alpha_{7}$, and $a_{2}, \ldots, a_{12}$.

\subsubsection{Loop Closure}

The double butterfly linkage has three loops. The isotropic form of the loop closure conditions for the double butterfly linkage can be written as 


$$
\begin{aligned}
& g_{1}:=a_{1} \mathbf{T}_{1}+a_{2} \mathbf{T}_{2}+a_{3} \mathbf{T}_{3}+a_{4} \mathbf{T}_{4}+a_{5} \mathbf{T}_{5}=0, \\
& \bar{g}_{1}:=a_{1} \overline{\mathbf{T}}_{1}+a_{2} \overline{\mathbf{T}}_{2}+a_{3} \overline{\mathbf{T}}_{3}+a_{4} \overline{\mathbf{T}}_{4}+a_{5} \overline{\mathbf{T}}_{5}=0, \\
& g_{2}:=a_{1} \mathbf{T}_{1}+b_{6} \mathbf{T}_{2}+a_{7} \mathbf{T}_{7}+a_{8} \mathbf{T}_{8}+b_{9} \mathbf{T}_{4}+a_{5} \mathbf{T}_{5}=0, \\
& \bar{g}_{2}:=a_{1} \overline{\mathbf{T}}_{1}+b_{6}^{\prime} \overline{\mathbf{T}}_{2}+a_{7} \overline{\mathbf{T}}_{7}+a_{8} \overline{\mathbf{T}}_{8}+b_{9}^{\prime} \overline{\mathbf{T}}_{4}+a_{5} \overline{\mathbf{T}}_{5}=0, \\
& g_{3}:=a_{1} \mathbf{T}_{1}+b_{6} \mathbf{T}_{2}+b_{10} \mathbf{T}_{7}+a_{11} \mathbf{T}_{11}+a_{12} \mathbf{T}_{12}=0, \\
& \bar{g}_{3}:=a_{1} \overline{\mathbf{T}}_{1}+b_{6}^{\prime} \overline{\mathbf{T}}_{2}+b_{10}^{\prime} \overline{\mathbf{T}}_{7}+a_{11} \overline{\mathbf{T}}_{11}+a_{12} \overline{\mathbf{T}}_{12}=0, \\
& h_{j}:=\mathbf{T}_{j} \overline{\mathbf{T}}_{j}-1=0, \quad j=2,3,4,7,8,11 .
\end{aligned}
$$

As before, all of the $a_{j}$ are real link lengths, but $b_{6}, b_{9}, b_{10}$ are the complex stretch rotations to properly model the ternary links. That is, $b_{6}=a_{6}\left(\cos \alpha_{2}+i \sin \alpha_{2}\right), b_{9}=a_{9}\left(\cos \alpha_{4}+i \sin \alpha_{4}\right), b_{10}=$ $a_{10}\left(\cos \alpha_{7}+i \sin \alpha_{7}\right)$, and $b_{6}^{\prime}, b_{9}^{\prime}, b_{10}^{\prime}$ are the complex conjugates of $b_{6}, b_{9}, b_{10}$. The system of twelve equations in Eqs. 4.20 describes a curve in the twelve-dimensional space of $\left\{\mathbf{T}_{2}, \mathbf{T}_{3}, \mathbf{T}_{4}, \mathbf{T}_{7}\right.$, $\left.\mathbf{T}_{8}, \mathbf{T}_{11}, \overline{\mathbf{T}}_{2}, \overline{\mathbf{T}}_{3}, \overline{\mathbf{T}}_{4}, \overline{\mathbf{T}}_{7}, \overline{\mathbf{T}}_{8}, \overline{\mathbf{T}}_{11}\right\}$. In the numerical examples that follow, the values used for the physical parameters are: $a_{2}=5.0, a_{3}=2.0, a_{4}=2.0, a_{5}=4.0, a_{6}=7.0, a_{7}=5.0$, $a_{8}=2.0, a_{9}=2.0, a_{1} 0=9.0, a_{1} 1=7.0, a_{1} 2=7.0, \theta_{5}=3.9168, \theta_{12}=3.1416, \alpha_{4}=1.0472$, $\alpha_{2}=0.3803$ and $\alpha_{7}=0.4510$. 


\subsubsection{Forward Kinematics}

The forward kinematic problem considers $p=a_{1}$ fixed, the passive joint variables are determined for a given input $\theta_{1}$. Some variables can be eliminated from Eqs. (4.20) to facilitate solution,

$$
\begin{aligned}
& \mathbf{R}_{3}:=-a_{3} \mathbf{T}_{3}=a_{1} \mathbf{T}_{1}+a_{2} \mathbf{T}_{2}+a_{4} \mathbf{T}_{4}+a_{5} \mathbf{T}_{5} \\
& \overline{\mathbf{R}}_{3}:=-a_{3} \overline{\mathbf{T}}_{3}=a_{1} \overline{\mathbf{T}}_{1}+a_{2} \overline{\mathbf{T}}_{2}+a_{4} \overline{\mathbf{T}}_{4}+a_{5} \overline{\mathbf{T}}_{5} \\
& \mathbf{R}_{8}:=-a_{8} \mathbf{T}_{8}=a_{1} \mathbf{T}_{1}+b_{6} \mathbf{T}_{2}+a_{7} \mathbf{T}_{7}+b_{9} \mathbf{T}_{4}+a_{5} \mathbf{T}_{5} \\
& \overline{\mathbf{R}}_{8}:=-a_{8} \overline{\mathbf{T}}_{8}=a_{1} \overline{\mathbf{T}}_{1}+b_{6}^{\prime} \overline{\mathbf{T}}_{2}+a_{7} \overline{\mathbf{T}}_{7}+b_{9} \overline{\mathbf{T}}_{4}+a_{5} \overline{\mathbf{T}}_{5} \\
& \mathbf{R}_{11}:=-a_{11} \mathbf{T}_{11}=a_{1} \mathbf{T}_{1}+b_{6} \mathbf{T}_{2}+b_{10} \mathbf{T}_{7}+a_{12} \mathbf{T}_{12} \\
& \overline{\mathbf{R}}_{11}:=-a_{11} \mathbf{T}_{11}=a_{1} \overline{\mathbf{T}}_{1}+b_{6}^{\prime} \overline{\mathbf{T}}_{2}+b_{10}^{\prime} \overline{\mathbf{T}}_{7}+a_{12} \overline{\mathbf{T}}_{12}
\end{aligned}
$$

Accordingly, for $a_{i} \neq 0, i=3,8,11, a_{i}^{2} h_{i}=0$ is formed to achieve

$$
\begin{array}{r}
H_{3}:=\mathbf{R}_{3} \overline{\mathbf{R}}_{3}-a_{3}^{2}=0, \\
H_{8}:=\mathbf{R}_{8} \overline{\mathbf{R}}_{8}-a_{8}^{2}=0, \\
H_{11}:=\mathbf{R}_{11} \overline{\mathbf{R}}_{11}-a_{11}^{2}=0 .
\end{array}
$$

The identities corresponding to the remaining the joint valuables are

$$
\begin{aligned}
& h_{2}:=\mathbf{T}_{2} \overline{\mathbf{T}}_{2}-1=0, \\
& h_{4}:=\mathbf{T}_{4} \overline{\mathbf{T}}_{4}-1=0, \\
& h_{7}:=\mathbf{T}_{7} \overline{\mathbf{T}}_{7}-1=0,
\end{aligned}
$$

For a given design, the solution of this system of six Eqs. (4.22)-(4.27) in the six unknowns, separated into two-homogeneous variable groups $\left\{\mathbf{T}_{2}, \mathbf{T}_{4}, \mathbf{T}_{7}\right\},\left\{\overline{\mathbf{T}}_{2}, \overline{\mathbf{T}}_{4}, \overline{\mathbf{T}}_{7}\right\}$ is the motion curve of the linkage. As before, "real" solutions are those for which $\left|\mathbf{T}_{2}\right|=\left|\mathbf{T}_{4}\right|=\left|\mathbf{T}_{7}\right|=1$. At any 
point on the curve, the remaining angles can be solved by using

$$
\begin{array}{r}
\mathbf{T}_{3}=\mathbf{R}_{3} / a_{3}, \\
\mathbf{T}_{8}=\mathbf{R}_{8} / a_{8}, \\
\mathbf{T}_{11}=\mathbf{R}_{11} / a_{11} .
\end{array}
$$

\subsubsection{Singularity Points}

The singularity condition for double butterfly linkage is

$$
D:=\operatorname{det}\left[\begin{array}{lll}
\frac{\partial H_{3}}{\partial \theta_{2}} & \frac{\partial H_{3}}{\partial \theta_{4}} & \frac{\partial H_{3}}{\partial \theta_{7}} \\
\frac{\partial H_{8}}{\partial \theta_{2}} & \frac{\partial H_{8}}{\partial \theta_{4}} & \frac{\partial H_{8}}{\partial \theta_{7}} \\
\frac{\partial H_{11}}{\partial \theta_{2}} & \frac{\partial H_{11}}{\partial \theta_{4}} & \frac{\partial H_{11}}{\partial \theta_{7}}
\end{array}\right]=0 .
$$

Note that $\frac{\partial H_{3}}{\partial \theta_{7}}=0$ since $\mathbf{T}_{7}$ does not appear in $\mathbf{R}_{3}$, and $\frac{\partial H_{11}}{\partial \theta_{4}}=0$ since $\mathbf{T}_{4}$ does not appear in $\mathbf{R}_{8}$. The singularity points for double butterfly linkage are given by a system of equations consisting of the loop closure conditions Eqs. (4.22)-(4.27) along with Eq. (4.31), To find singularity points at a given value of $a_{1}$, the system involves the following variable groups $\left\{\mathbf{T}_{1}, \mathbf{T}_{2}, \mathbf{T}_{4}, \mathbf{T}_{7}\right\},\left\{\overline{\mathbf{T}}_{1}, \overline{\mathbf{T}}_{2}, \overline{\mathbf{T}}_{4}, \overline{\mathbf{T}}_{7}\right\}$.

\subsubsection{Motion Curve}

A trace of the motion curve with $a_{1}=0.198$ is projected onto the $\theta_{1}-\theta_{2}$ plane and shown in Fig. 4.16. This linkage has six circuits, with no singularities and six GIs for all values of $\theta_{1}$. That is, the linkage can be assembled into six different configurations all having continuously rotating cranks. A trace of the motion curve with a slightly longer $a_{1}=0.40$ is projected onto the $\theta_{1}-\theta_{2}$ plane and shown in Fig. 4.17. This linkage has seven circuits, six of which have continously rotating cranks. Additionally, the seventh circuit exhibits two singularity points. Thus, eight GIs exist for $-1.5768<\theta_{1}<0.3707$. A trace of the motion curve with a longer $a_{1}=2.50$ is projected onto the $\theta_{1}-\theta_{2}$ plane and shown in Fig. 4.18. This linkage has six circuits, one of which has a continously rotating crank. Of particular note is a circuit, where the linkage is able to rotate greater than one full revolution between singularities. This feature is seen as the " $U$ " shape observed and noted in 


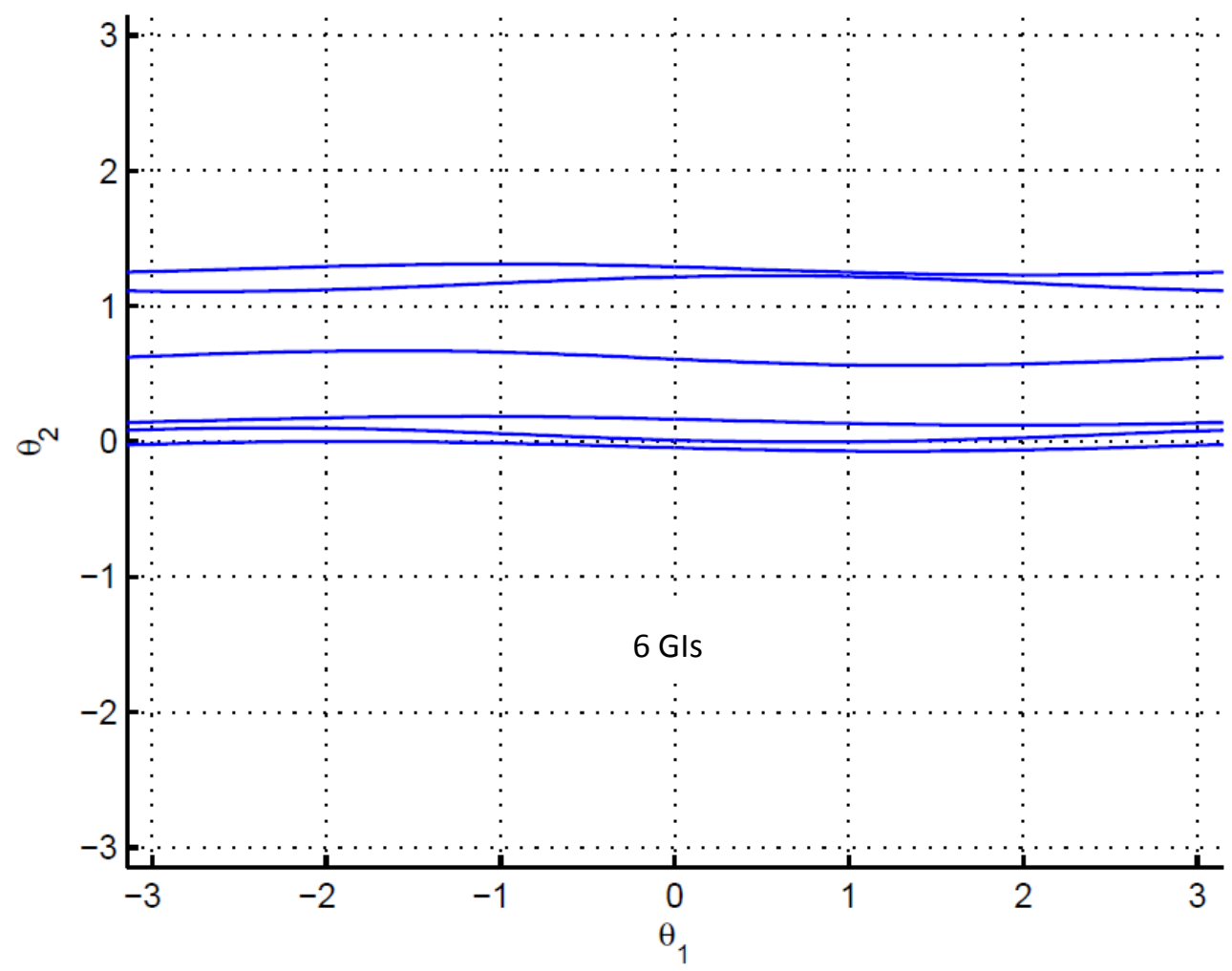

Figure 4.16: Trace of the motion curve for the double butterfly linkage, with $a_{1}=0.198$, projected onto the $\theta_{1}-\theta_{2}$ plane.

the motion trace of Fig. 4.18. Examples of this net-zero, singularity-free actuation that places the linkage into a different GI were identified for Stephenson III linkages in the previous section and in [1]. Those cases showed an "S" shape in the motion curve trace.

The final trace of the motion curve of a new double butterfly linkage with different parameters is projected onto the $\theta_{1}-\theta_{2}$ plane and shown in Fig. 4.19. This linkage has five circuits, none of which has a continously rotating crank. Of note is a circuit, where the linkage is also able to rotate greater than one full revolution between singularities. Again, a net-zero, singularity-free actuation 


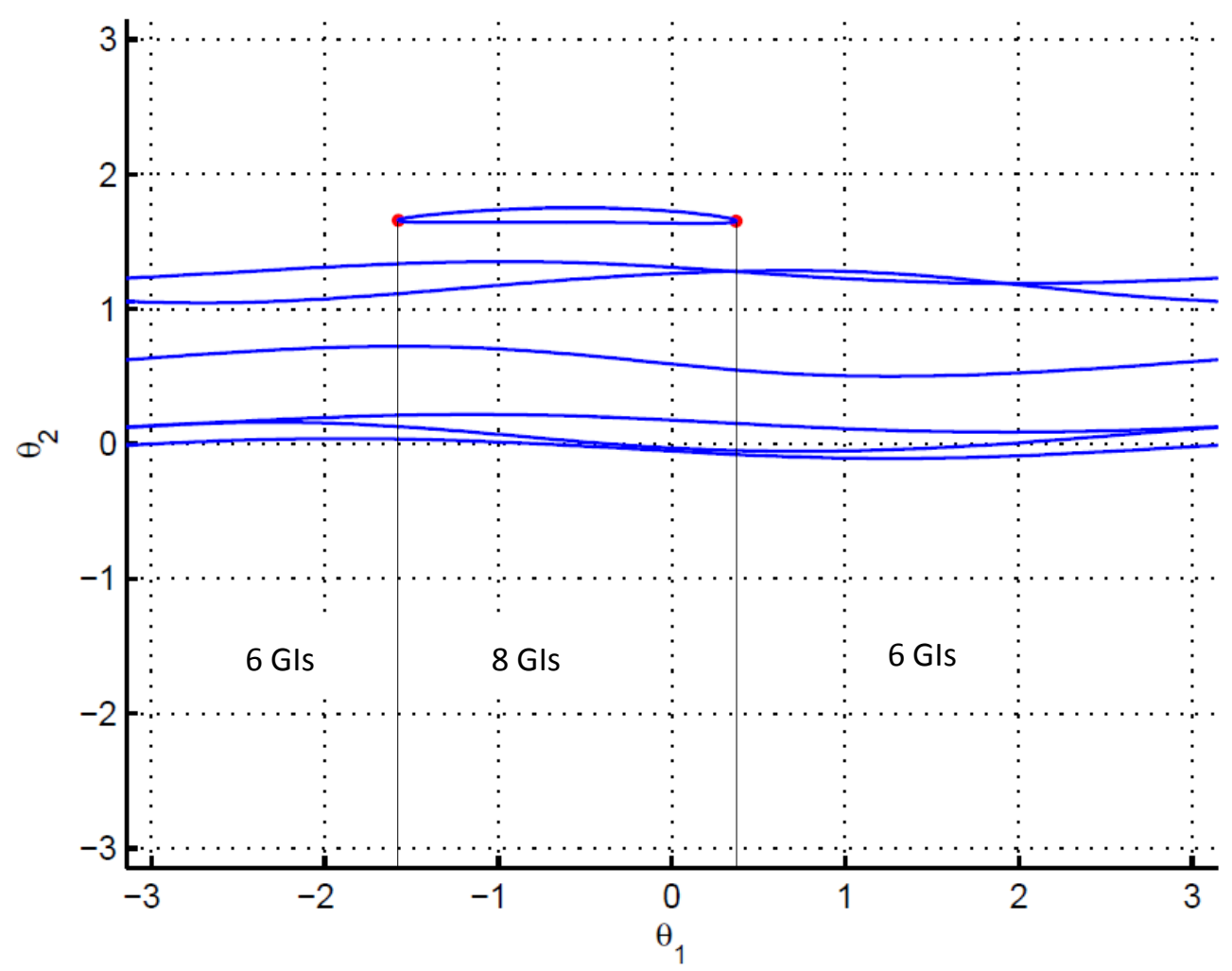

Figure 4.17: Trace of the motion curve for the double butterfly linkage, with $a_{1}=0.40$, projected onto the $\theta_{1}-\theta_{2}$ plane.

that places the linkage into a different GI is identified. However, this appears as a closed curve where the turning points (singulaties) are separated by more than $360^{\circ}$.

\subsubsection{Critical Points}

The system to solve for the critical points of double butterfly linkage when $a_{1}$ is considered the variable design parameter consists of Eqs. (4.22)-(4.27),(4.31), and

$$
E:=\operatorname{det}\left[\begin{array}{cccc}
\frac{\partial H_{3}}{\partial \theta_{1}} & \frac{\partial H_{3}}{\partial \theta_{2}} & \frac{\partial H_{3}}{\partial \theta_{4}} & \frac{\partial H_{3}}{\partial \theta_{7}} \\
\frac{\partial H_{8}}{\partial \theta_{1}} & \frac{\partial H_{8}}{\partial \theta_{2}} & \frac{\partial H_{8}}{\partial \theta_{4}} & \frac{\partial H_{8}}{\partial \theta_{7}} \\
\frac{\partial H_{11}}{\partial \theta_{1}} & \frac{\partial H_{11}}{\partial \theta_{2}} & \frac{\partial H_{11}}{\partial \theta_{4}} & \frac{\partial H_{11}}{\partial \theta_{7}} \\
\frac{\partial D}{\partial \theta_{1}} & \frac{\partial D}{\partial \theta_{2}} & \frac{\partial D}{\partial \theta_{4}} & \frac{\partial D}{\partial \theta_{7}}
\end{array}\right]=0 .
$$




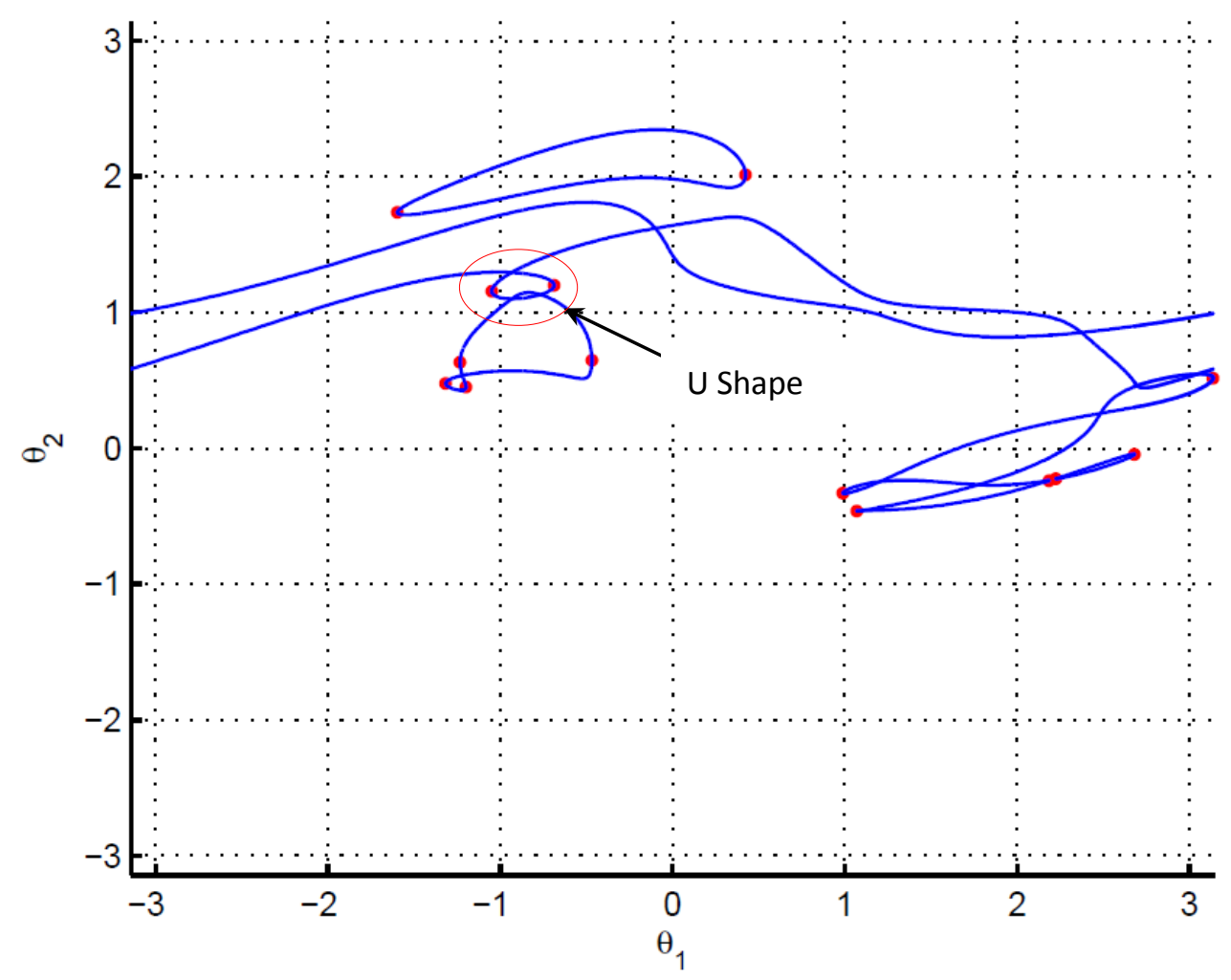

Figure 4.18: Trace of the motion curve for the double butterfly linkage, with $a_{1}=2.50$, projected onto the $\theta_{1}-\theta_{2}$ plane.

Note that $\frac{\partial H_{3}}{\partial \theta_{7}}=0$ since $\mathbf{T}_{7}$ does not appear in $\mathbf{R}_{3}$, and $\frac{\partial H_{11}}{\partial \theta_{4}}=0$ since $\mathbf{T}_{4}$ does not appear in $\mathbf{R}_{8}$. The system involves the following variables groups: $a_{1},\left\{\mathbf{T}_{1}, \mathbf{T}_{2}, \mathbf{T}_{4}, \mathbf{T}_{7}\right\},\left\{\overline{\mathbf{T}}_{1}, \overline{\mathbf{T}}_{2}, \overline{\mathbf{T}}_{4}, \overline{\mathbf{T}}_{7}\right\}$. However, the $4 \times 4$ matrix in Eq. (4.32) is complicated and its determinant is more difficult to calculate than the examples presented above. A new approach to simplify the calculation is to combine the design parameter variable $a_{1}$ and its angle $\mathbf{T}_{1}, \overline{\mathbf{T}}_{1}$ by defining $\mathbf{X}_{1}=a_{1} \mathbf{T}_{1}$ and $\overline{\mathbf{X}}_{1}=$ $a_{1} \overline{\mathbf{T}}_{1}$. Accordingly, the equation $\mathbf{T}_{1} \overline{\mathbf{T}}_{1}-1=0$ is eliminated from the system. The resulting variables groups are $\left\{\mathbf{X}_{1}, \mathbf{T}_{2}, \mathbf{T}_{4}, \mathbf{T}_{7}\right\},\left\{\overline{\mathbf{X}}_{1}, \overline{\mathbf{T}}_{2}, \overline{\mathbf{T}}_{4}, \overline{\mathbf{T}}_{7}\right\}$. Thus, this method reduces one variable 


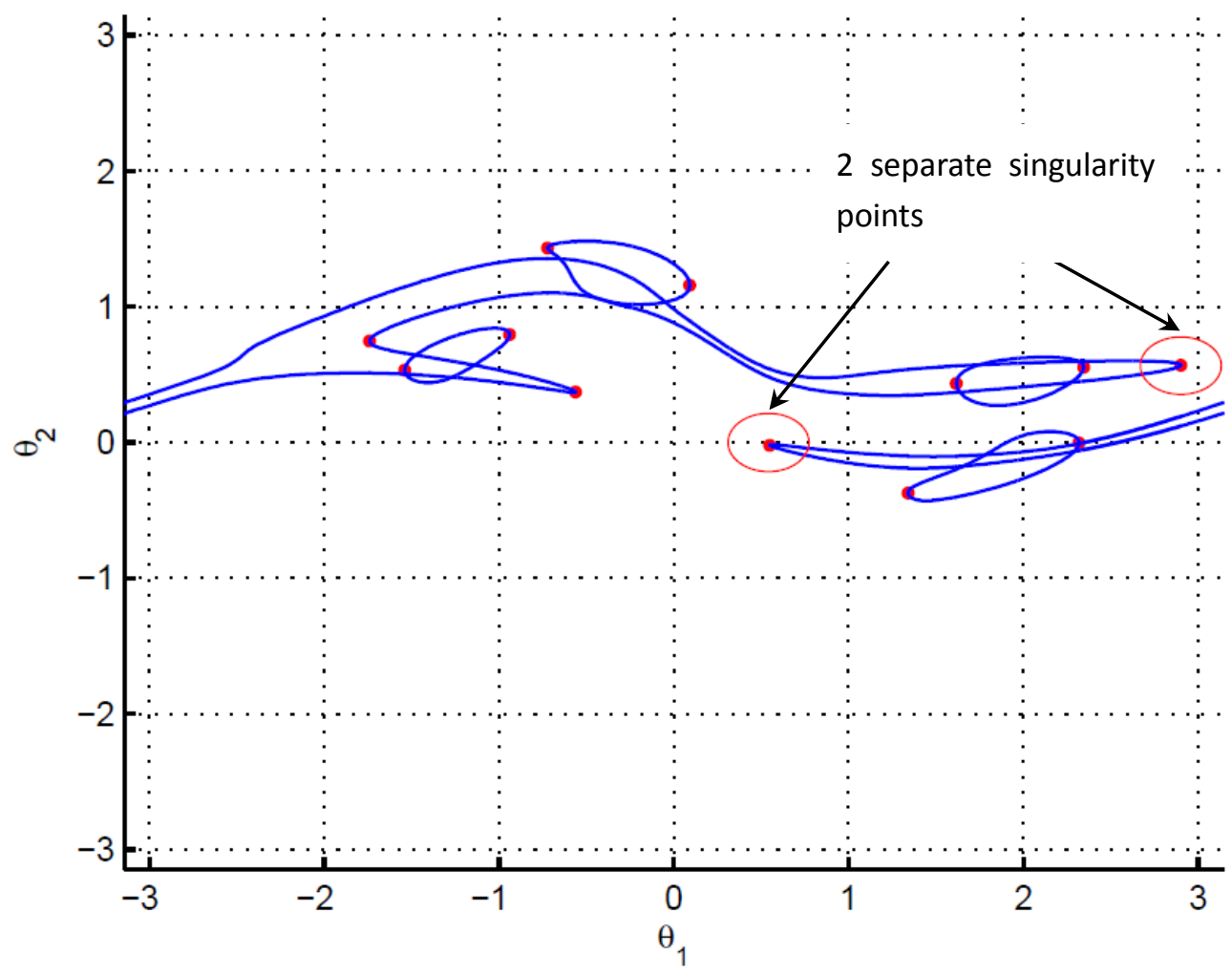

Figure 4.19: Trace of the motion curve for another double butterfly linkage with different parameters, projected onto the $\theta_{1}-\theta_{2}$ plane.

and one equation from system. The design parameter at critical points is determined as,

$$
a_{1}=\sqrt{\mathbf{X}_{1}^{2}+\overline{\mathbf{X}}_{1}^{2}}
$$

\subsubsection{Singularity Trace}

The singularity trace of double butterfly linkage with a variable $a_{1}$ is convoluted and shown in Fig. 4.20. As before, the singularity trace separates the plot into regions having the same number of GIs. Solving the forward kinematic problem for one sample point within each zone determines the 


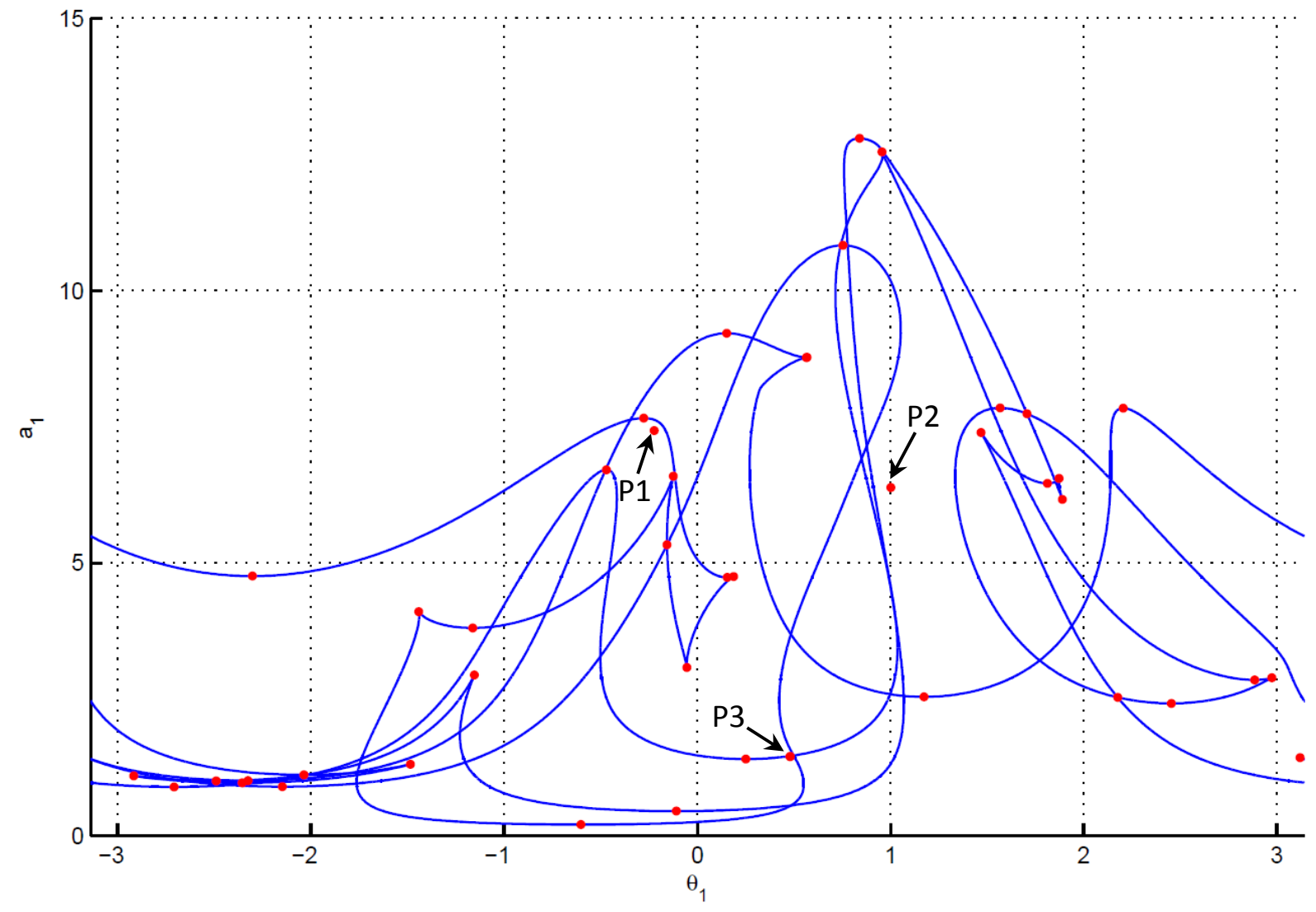

Figure 4.20: Double butterfly linkage singularity trace with respect to $a_{1}$.

number in GIs in that entire zone. Additionally, sampling the motion curve between critical points that appear as smooth extrema determines the number of circuits. There are three isolated critical points $P 1, P 2$ and $P 3$ which do not lie on the singularity curve in Fig. 4.20. These critical points identify where a GI of the double butterfly linkage will lose a DOF and become a fixed structure as indicated in Fig. 4.21.

A singularity trace of the double butterfly linkage with a variable $a_{3}$ is also convoluted and shown in Fig. 4.22. Similar to the singularity trace with respect to $a_{1}$, there are many cusps, and 


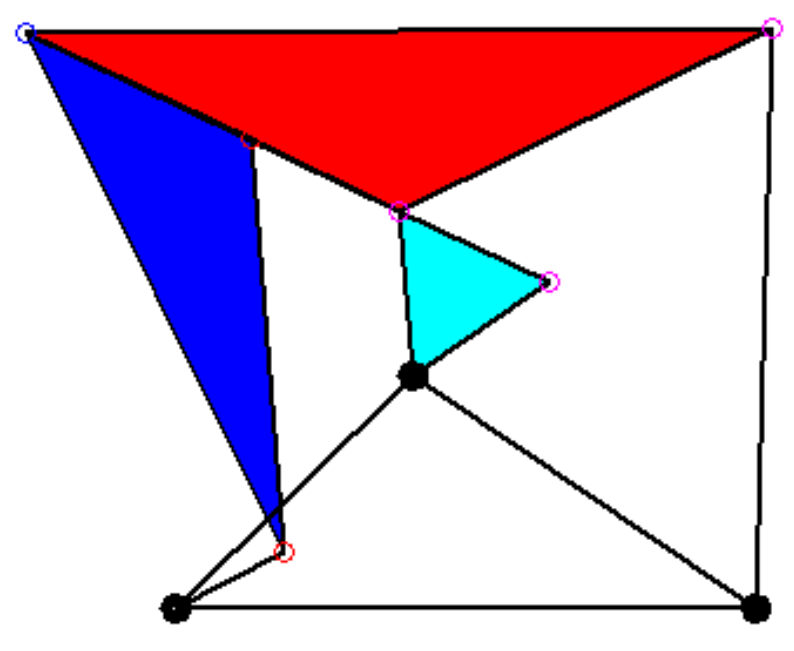

Figure 4.21: Double butterfly isolated critical position at P3.

the cusps are related to a net-zero, singularity free activation sequence that places mechanism into a different GI.

\subsection{Spherical Four-Bar Linkage}

For the spherical four-bar linkage shown in Fig. 4.23, $x=\theta_{1}$ is considered the input angle and the design parameter is designated as $p=\alpha$. The remaining joint variables are $y=\left\{\theta_{2}, \theta_{3}, \theta_{4}\right\}$. The physical parameters are $\beta, \gamma$ and $\eta$. In the numerical examples that follow, the values used for the physical parameters are: $\alpha=\pi / 3, \beta=\pi / 4, \eta=\pi / 2$, and $\gamma=\pi / 2.5$. Because the spherical four-bar linkage has spatial loop closure which is different from the planar examples, rotation matrix has been introduced here to generate the forward kinematics equations of this mechanism.

\subsubsection{Forward Kinematics}

According to Fig. 4.23, using Eq. (2.19), the forward kinematics conditions become

$$
\left[X\left(\theta_{1}\right)\right][Z(\alpha)]\left[X\left(\theta_{2}\right)\right][Z(\eta)]=[Z(\gamma)]\left[X\left(\theta_{4}\right)\right][Z(\beta)]\left[X\left(\theta_{3}\right)\right]
$$




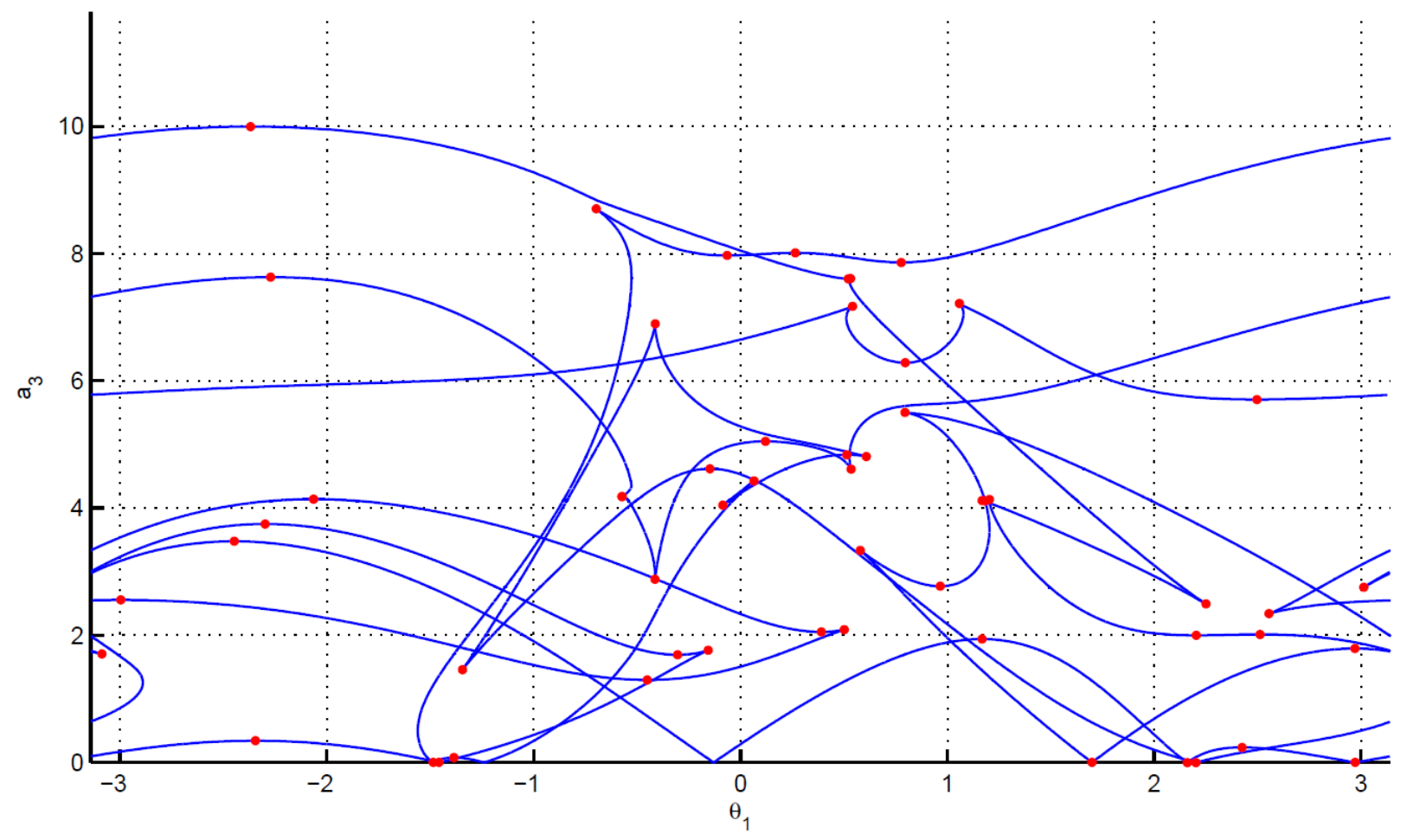

Figure 4.22: Double butterfly linkage singularity trace with respect to $a_{3}$.

or

$$
\left[A_{\text {in }}\right]=\left[A_{\text {out }}\right]
$$

From Eq. (4.35) we can have 9 equations,

$$
\left[\begin{array}{lll}
f_{1} & f_{2} & f_{3} \\
f_{4} & f_{5} & f_{6} \\
f_{7} & f_{8} & f_{9}
\end{array}\right]=\left[A_{\text {in }}\right]-\left[A_{\text {out }}\right]=\left[\begin{array}{lll}
0 & 0 & 0 \\
0 & 0 & 0 \\
0 & 0 & 0
\end{array}\right] .
$$

To convert the system to being algebraic, the joint variables $\left\{\theta_{2}, \theta_{3}, \theta_{4}\right\}$ are divided into six variables as $\left\{\cos \theta_{2}, \sin \theta_{2}, \cos \theta_{3}, \sin \theta_{3}, \cos \theta_{4}, \sin \theta_{4}\right\}$. Bertini can solve equations with less number of variables than the number of equations. 


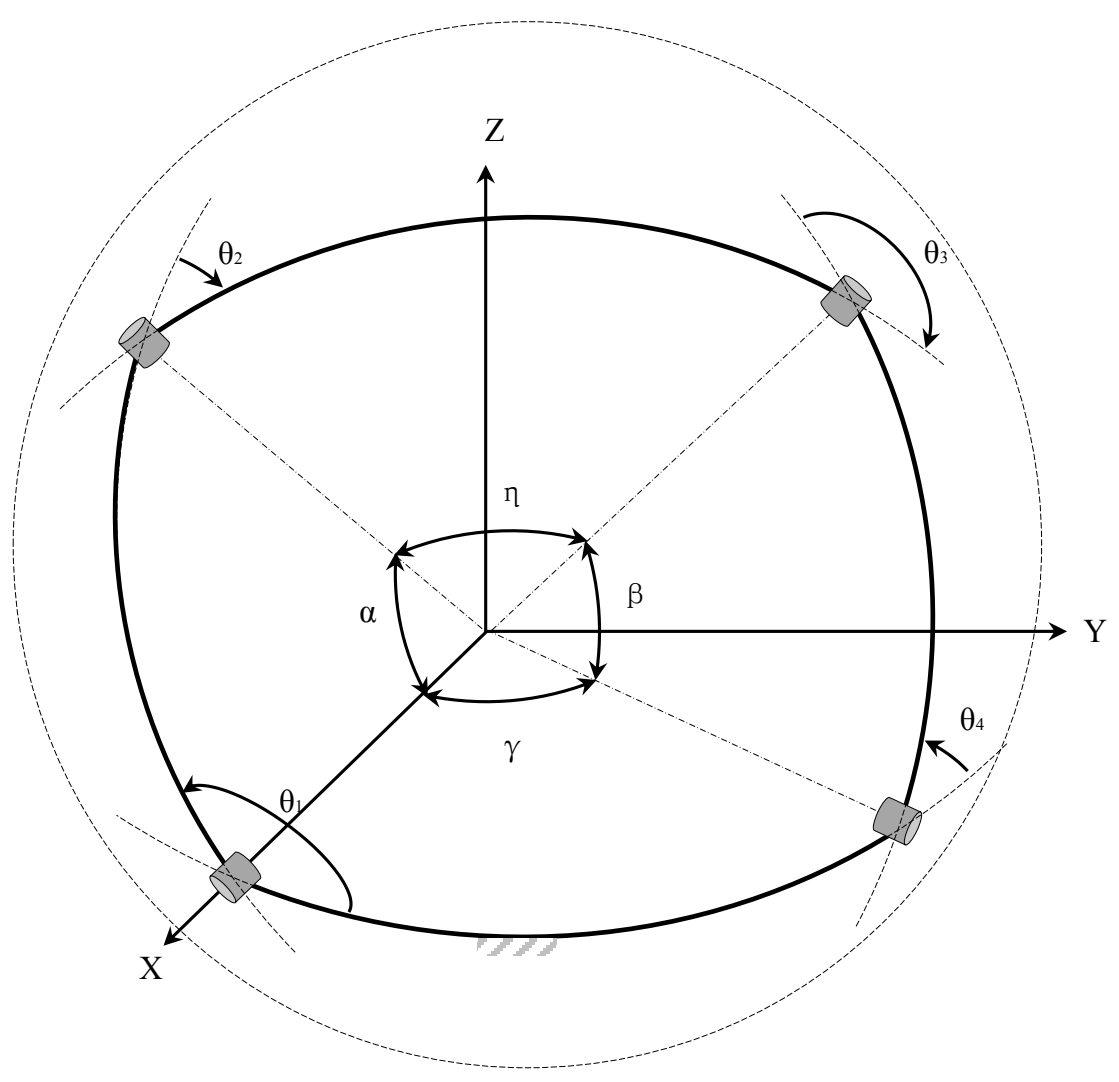

Figure 4.23: Spherical Four-Bar linkage.

For a given design, the solutions of this system of nine Eqs. (4.36) in the six unknowns, separated into three variable groups $\left\{\cos \theta_{2}, \sin \theta_{2}\right\},\left\{\cos \theta_{3}, \sin \theta_{3}\right\},\left\{\cos \theta_{4}, \sin \theta_{4}\right\}$, is the motion curve of the linkage. Different from before, "real" solutions are those for which satisfy $\cos ^{2} \theta_{2}+\sin ^{2} \theta_{2}=$ $\cos ^{2} \theta_{3}+\sin ^{2} \theta_{3}=\cos ^{2} \theta_{4}+\sin ^{2} \theta_{4}=1$.

Another approach to solve forward kinematics of this mechanism is that for any non-zero value $a$ along the $x$-axis in Fig. 4.23, there exists

$$
\left[X\left(\theta_{1}\right)\right][Z(\alpha)]\left[X\left(\theta_{2}\right)\right][Z(\eta)]\left\{\begin{array}{l}
a \\
0 \\
0
\end{array}\right\}=[Z(\gamma)]\left[X\left(\theta_{4}\right)\right][Z(\beta)]\left\{\begin{array}{l}
a \\
0 \\
0
\end{array}\right\},
$$

we can have three equations with four variables $\left\{\cos \theta_{2}, \sin \theta_{2}, \cos \theta_{4}, \sin \theta_{4}\right\}$ as

$$
\left\{\begin{array}{l}
f_{1} \\
f_{2} \\
f_{3}
\end{array}\right\}=\left(\left[X\left(\theta_{1}\right)\right][Z(\alpha)]\left[X\left(\theta_{2}\right)\right][Z(\eta)]-[Z(\gamma)]\left[X\left(\theta_{4}\right)\right][Z(\beta)]\right)\left\{\begin{array}{l}
1 \\
0 \\
0
\end{array}\right\}=\left\{\begin{array}{l}
0 \\
0 \\
0
\end{array}\right\} .
$$


Because the four variables $\left\{\cos \theta_{2}, \sin \theta_{2}, \cos \theta_{4}, \sin \theta_{4}\right\}$ are treated separately, but are derived from $\theta_{2}$ and $\theta_{4}$, they must satisfy the identity conditions as

$$
f_{4}=\cos ^{2} \theta_{2}+\sin ^{2} \theta_{2}-1=0
$$

and

$$
f_{5}=\cos ^{2} \theta_{4}+\sin ^{2} \theta_{4}-1=0
$$

Combining with Eq. (4.38), we can use either of these two identity equations as the fourth equation of the system to solve the forward kinematics problem of spherical four-bar.

We can determine $\theta_{2}$ and $\theta_{4}$ from the values of $\left\{\cos \theta_{2}, \sin \theta_{2}, \cos \theta_{4}, \sin \theta_{4}\right\}$. To complete the position analysis, the joint variables $\left\{\cos \theta_{3}, \sin \theta_{3}\right\}$ can be obtained by using the inverse kinematic formulation as shown in Sec. 2.6.1,

$$
\left[X\left(\theta_{3}\right)\right]=\left([Z(\gamma)]\left[X\left(\theta_{4}\right)\right][Z(\beta)]\right)^{-1}\left[X\left(\theta_{1}\right)\right][Z(\alpha)]\left[X\left(\theta_{2}\right)\right][Z(\eta)]
$$

\subsubsection{Singularity Points}

For the spherical 4R linkage, we use the method of treating sin and cos as separate variables to solve the forward kinematics. Keeping with this approach, the singularity condition is

$$
D:=\operatorname{det}\left[\begin{array}{cccc}
\frac{\partial f_{1}}{\partial \cos \theta_{2}} & \frac{\partial f_{1}}{\partial \sin \theta_{2}} & \frac{\partial f_{1}}{\partial \cos \theta_{4}} & \frac{\partial f_{1}}{\partial \sin \theta_{4}} \\
\frac{\partial f_{2}}{\partial \cos \theta_{2}} & \frac{\partial f_{2}}{\partial \sin \theta_{2}} & \frac{\partial f_{2}}{\partial \cos \theta_{4}} & \frac{\partial f_{2}}{\partial f_{2} \theta_{4}} \\
\frac{\partial f_{3}}{\partial \cos \theta_{2}} & \frac{\partial f_{3}}{\partial \sin \theta_{2}} & \frac{\partial f_{3}}{\partial \cos \theta_{4}} & \frac{\partial f_{3}}{\partial \sin \theta_{4}} \\
\frac{\partial f_{4}}{\partial \cos \theta_{2}} & \frac{\partial f_{4}}{\partial \sin \theta_{2}} & \frac{\partial f_{4}}{\partial \cos \theta_{4}} & \frac{\partial f_{4}}{\partial \sin \theta_{4}}
\end{array}\right]=0 .
$$

Eqs. (4.38) and (4.39) must both be satisfied to solve for singularity points of this mechanism.

It is noted that spherical four-bar linkage has some common properties with planar four-bar linkage. The singularity position of the spherical four-bar linkage shown in Fig. 4.24 is similar to the singularity position of planar four-bar mechanism in Fig. 2.6 shown in Sec. 2.4. When the 


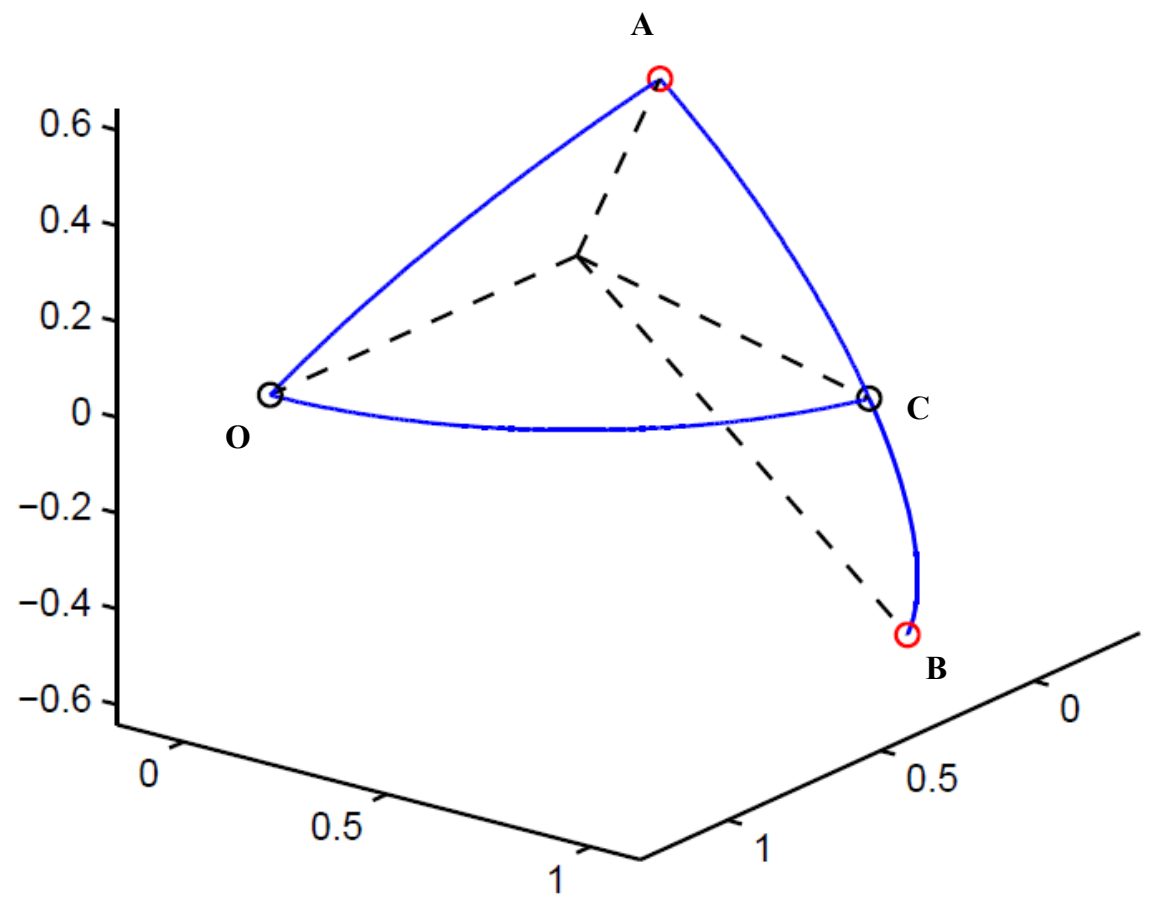

Figure 4.24: Spherical four-bar linkage singularity position.

pivots $\mathbf{A}, \mathbf{B}$ and $\mathbf{C}$ lie along the same arc, the input link $\mathbf{O A}$ cannot actuate the linkage and the links become locked.

\subsubsection{Motion Curve}

A trace of a motion curve with $\alpha=1.5$ is projected onto the $\theta_{1}-\theta_{2}$ plane and shown in Fig. 4.25. Note that the linkage has two circuits and four singularity points at $\theta_{1}=-0.7636,-2.4471,0.7636,2.4471$. Zero GIs exits for $\theta_{1}<-2.4471,-0.7636<\theta_{1}<0.7636$ and $\theta_{1}>2.4471$. Two GIs exist for $-2.4471<\theta_{1}<-0.7636$ and $0.7636<\theta_{1}<2.4471$. 


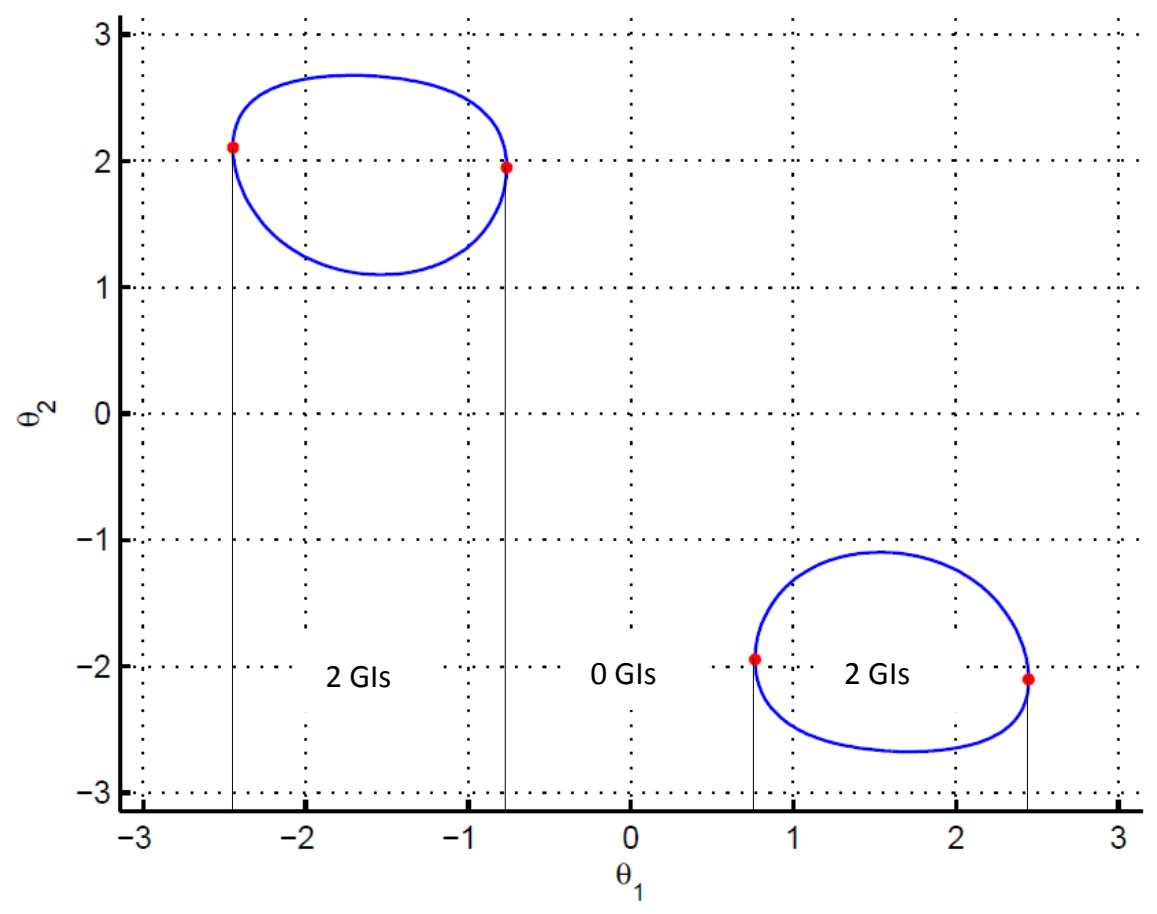

Figure 4.25: Trace of the motion curve for the spherical four-bar linkage, with $\alpha=1.5$, projected onto the $\theta_{1}-\theta_{2}$ plane.

\subsubsection{Critical Points}

The system used to determine the critical points of the spherical four-bar linkage when $\alpha$ is considered the variable design parameter consists of Eqs. (4.37), (4.38),(4.39),(4.42),

$$
f_{5}=\cos \theta_{1}^{2}+\sin \theta_{1}^{2}-1=0
$$

and

$$
E:=\operatorname{det}\left[\begin{array}{lllllll}
\frac{\partial f_{1}}{\partial \cos \theta_{1}} & \frac{\partial f_{1}}{\partial \sin \theta_{1}} & \frac{\partial f_{1}}{\partial \cos \theta_{2}} & \frac{\partial f_{1}}{\partial \sin \theta_{2}} & \frac{\partial f_{1}}{\partial \cos \theta_{4}} & \frac{\partial f_{1}}{\partial \sin \theta_{4}} \\
\frac{\partial f_{2}}{\partial \cos \theta_{1}} & \frac{\partial f_{2}}{\partial \sin \theta_{1}} & \frac{\partial f_{2}}{\partial \cos \theta_{2}} & \frac{\partial f_{2}}{\partial \sin \theta_{2}} & \frac{\partial f_{2}}{\partial \cos \theta_{4}} & \frac{\partial f_{2}}{\partial \sin \theta_{4}} \\
\frac{\partial f_{3}}{\partial \cos \theta_{1}} & \frac{\partial f_{3}}{\partial \sin \theta_{1}} & \frac{\partial f_{3}}{\partial \cos \theta_{2}} & \frac{\partial f_{3}}{\partial \sin \theta_{2}} & \frac{\partial f_{3}}{\partial \cos \theta_{4}} & \frac{\partial f_{3}}{\partial \sin \theta_{4}} \\
\frac{\partial f_{4}}{\partial \cos \theta_{1}} & \frac{\partial f_{4}}{\partial \sin \theta_{1}} & \frac{\partial f_{4}}{\partial \cos \theta_{2}} & \frac{\partial f_{4}}{\partial \sin \theta_{2}} & \frac{\partial f_{4}}{\partial \cos \theta_{4}} & \frac{\partial f_{4}}{\partial \sin \theta_{4}} \\
\frac{\partial f_{5}}{\partial \cos \theta_{1}} & \frac{\partial f_{5}}{\partial \sin \theta_{1}} & \frac{\partial f_{5}}{\partial \cos \theta_{2}} & \frac{\partial f_{5}}{\partial \sin \theta_{2}} & \frac{\partial f_{5}}{\partial \cos \theta_{4}} & \frac{\partial f_{5}}{\partial \sin \theta_{4}} \\
\frac{\partial D}{\partial \cos \theta_{1}} & \frac{\partial D}{\partial \sin \theta_{1}} & \frac{\partial D}{\partial \cos \theta_{2}} & \frac{\partial D}{\partial \sin \theta_{2}} & \frac{\partial D}{\partial \cos \theta_{4}} & \frac{\partial D}{\partial \sin \theta_{4}}
\end{array}\right]=0 .
$$




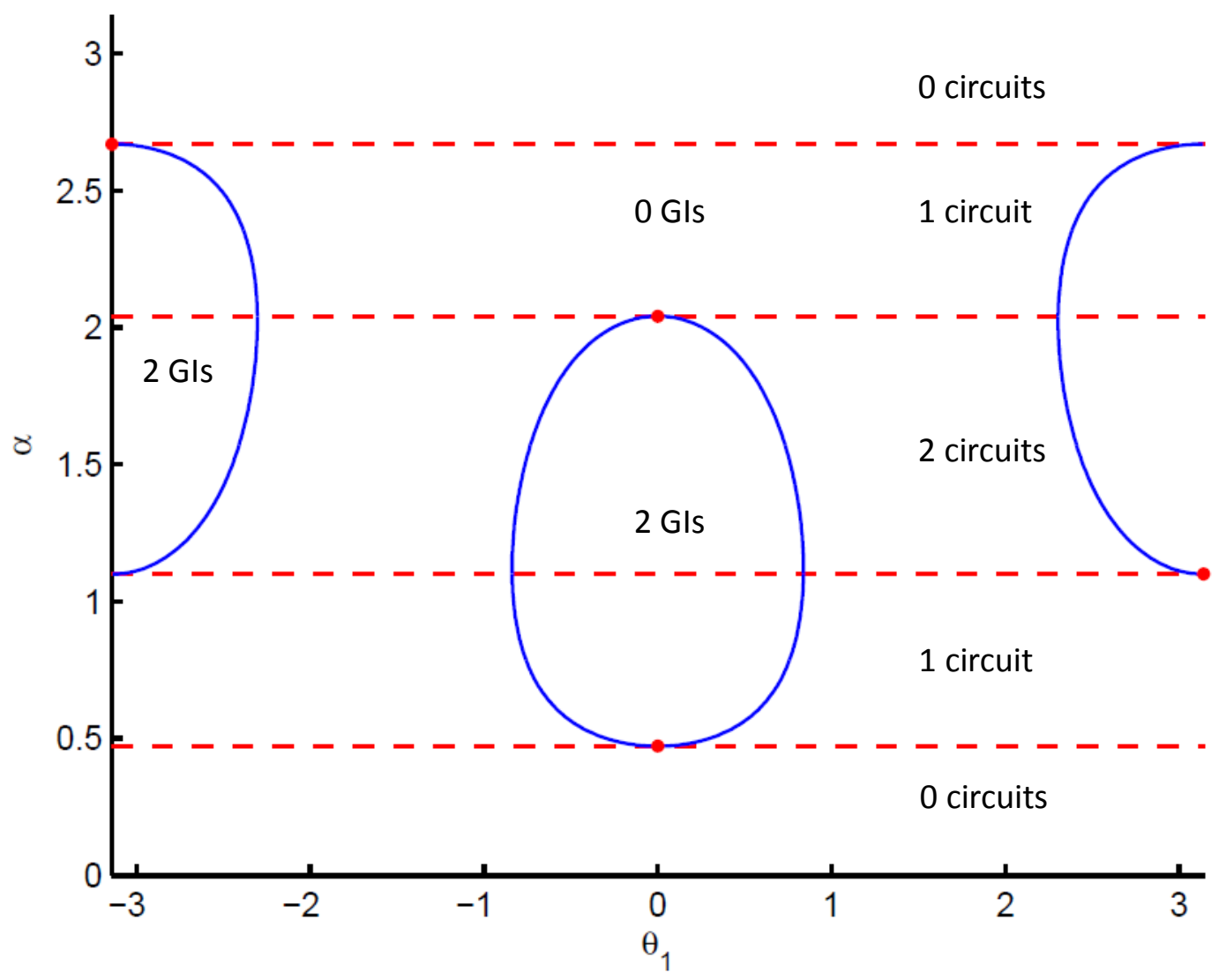

Figure 4.26: Projection of the spherical four-bar linkage singularity trace with respect to $\alpha$. Red circles mark the critical points. Regions of equal GIs and circuits are identified.

\subsubsection{Singularity Trace}

The singularity trace of spherical four-bar linkage with $\alpha$ as a variable is shown in Fig. 4.26. Solving the forward kinematic problem for one sample point within each region bounded by the singularity curve determines the number of GIs in that entire region. The number of GIs are noted in Fig. 4.26. And the number of circuits changes by one at each extrema are noted in Fig. 4.26. 


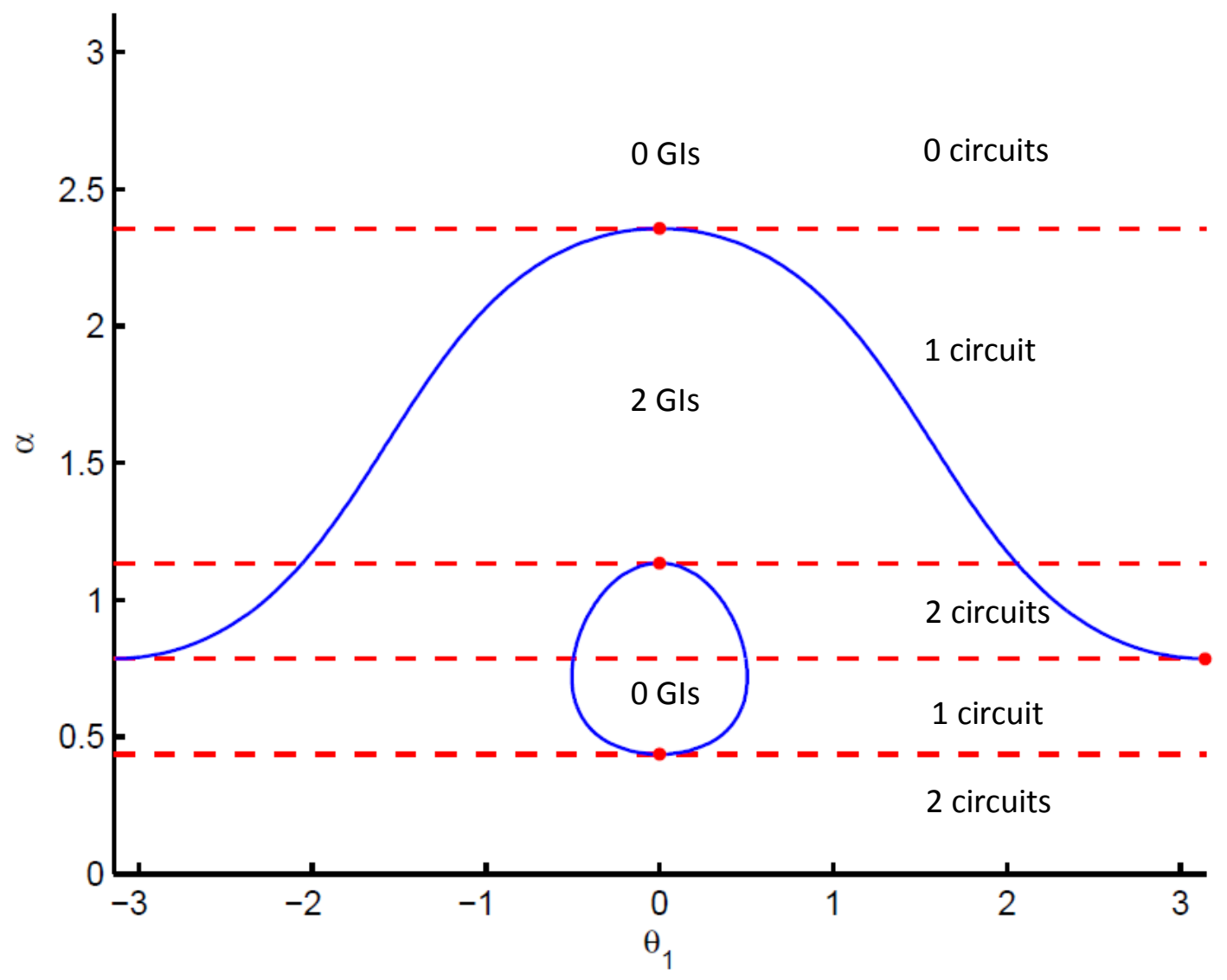

Figure 4.27: Projection of the spherical four-bar linkage singularity trace with respect to $\alpha$. Red circles mark the critical points. Regions of equal GIs and circuits are identified.

As discussed above, a spherical four-bar linkage is similar to a planar four-bar linkage. Designating $\alpha$ as the design variable and using the values for the physical parameters as: $\beta=0.6109$, $\eta=0.9599$, and $\gamma=0.7854$, this spherical four-bar linkage is in the same proportion as the parameters of the planar four-bar linkage as discussed in Sec. 3.2. The singularity trace of this spherical four-bar is shown in Fig. 4.27. This figure has similar properties with Fig. 3.3. 


\section{CHAPTER V}

\section{CONCLUSION AND FUTURE WORK}

\subsection{Conclusion}

This thesis illustrated numerous examples of a general method for computing the forward kinematics, singularity points, critical points, motion curve and singularity trace for single DOF, closedloop linkages with a designated input angle and one design parameter. The method was applied to the Watt II, Stephenson III and double butterfly planar linkages, and the spherical four-bar linkage. Singularity traces for each linkage were constructed and analyzed. Several instances of the Stephenson III and double-butterfly linkages where the input angle is able to rotate more than one revolution between singularities were illustrated. Instances presented where the singularity trace includes multiple coincident projections of the singularity curve. Additionally, isolated critical points were identified where the linkage configuration becomes a structure and loses the freedom to move.

\subsection{Future Work}

The RCCC linkage is a spatial four-bar mechanism with one DOF, which makes it an excellent linkage for the first usage of this new analysis method for spatial problems. As shown in Fig. 5.1, the RCCC linkage is composed of one revolute joint and three cylindrical joints. A cylindrical joint is a two DOF kinematic pair used in mechanisms. Cylindrical joints provide single-axis sliding as well as a single-axis of rotation. 


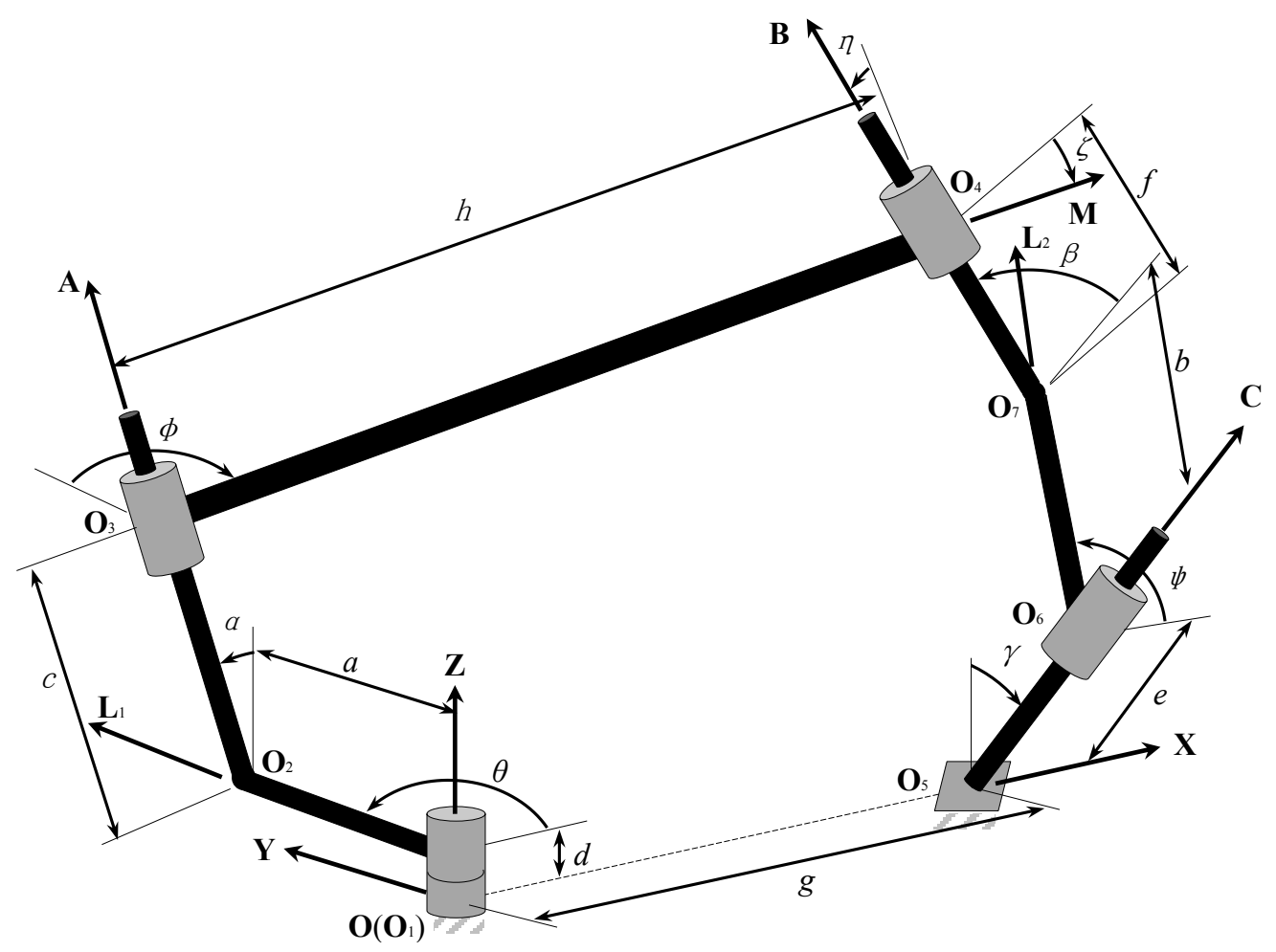

Figure 5.1: RCCC linkage.

For the RCCC linkage shown in Fig. 5.1, $x=\theta$ is considered the input angle. The joint and translation variables are $y=\{\phi, \psi, \zeta, c, e, f\}$. The physical joint parameters are $\alpha, \beta, \gamma, \eta$, and link length variables are $a, b, g, h$. In the numerical examples that follow, the values used for the physical parameters are: $\alpha=\pi / 2.5, \beta=\pi / 3, \gamma=\pi / 4, \eta=\pi / 5$ and $a=3.5, b=5.0, g=4.0$, $h=4.5$.

\subsubsection{Forward Kinematics}

Position the fixed frame $F$ with its origin at $\mathbf{O}$, its $z$-axis along $\mathbf{Z}$ and its $x$-axis along $\mathbf{X}$. Introduce the link frame $M_{1}$ with its origin at $\mathbf{O}_{1}$ which coincides with $\mathbf{O}$ and its $x$-axis aligned with $\mathbf{L}_{1}$. The input crank can be divided into two parts. The first part is the linkage between the 
input revolute joint and the orthogonal elbow of the crank. The second part is the linkage between the elbow and cylindrical joint. The displacement of the first part of the input crank is given by the screw displacement of $M_{1}$ along $\mathbf{Z}$ of distance $d=\left|\mathbf{O}-\mathbf{O}_{1}\right|=0$ and angle $\theta$ is measured from the $x$-axis of $F$ to $\mathbf{L}_{1}$. Let these joint parameters be assembled into the pair $\hat{\theta}=(\theta, d)$.

For the displacement of the second part of the input crank, attach the frame $M_{2}$ with its origin at $\mathbf{O}_{2}$, its $z$-axis along $\mathbf{A}$ and its $x$-axis aligned with $\mathbf{L}_{1}$. The displacement of the second part is the screw displacement of $M_{2}$ along $\mathbf{L}_{1}$ of distance $a=\left|\mathbf{O}_{1}-\mathbf{O}_{2}\right|$ is the length of the first part linkage and angle $\alpha$ is measured from the $z$-axis of $M_{1}$ to $\mathbf{A}$. Let these joint parameters be assembled into the pair $\hat{\alpha}=(\alpha, a)$.

The position of the coupler relative to the input crank is defined by attaching the frame $M_{3}$ with its origin at $\mathbf{O}_{3}$, its $z$-axis along $\mathbf{A}$, and its $x$-axis aligned with $\mathbf{M}$. The distance $c=\left|\mathbf{O}_{2}-\mathbf{O}_{3}\right|$ is the slider of the moving joint, and $\phi$ is its rotation angle measured from $\mathbf{L}_{1}$ to $\mathbf{M}$. The joint parameters are assembled into pairs, $\hat{\phi}=(\phi, c)$.

Similarly, the position of the cylindrical joint which connects the coupler and output crank is defined by attaching the frame $M_{4}$ with its origin at $\mathbf{O}_{4}$, its $z$-axis along $\mathbf{B}$, and its $x$-axis aligned with $\mathbf{M}$. The distance $h=\left|\mathbf{O}_{3}-\mathbf{O}_{4}\right|$ is the length of the coupler crank, and $\eta$ is its rotation angle measured from the $z$-axis of $M_{3}$ to $\mathbf{B}$. The joint parameters are assembled into pairs, $\hat{\eta}=(\eta, h)$.

These definitions yield the kinematics equations of the input crank $\mathbf{O A}$ as

$$
\left[D_{i n}\right]=[Z(\theta, d)][X(\alpha, a)][Z(\phi, c)][X(\eta, h)]
$$

We can attach link frame $M_{5}, M_{6}$ and $M_{7}$ on output crank in exactly the same way to obtain the kinematics equations for the position of the cylindrical joint connecting the coupler and output crank as

$$
\left[D_{\text {out }}\right]=[X(\gamma, g)][Z(\psi, e)][X(\beta, b)][Z(\zeta, f)]
$$


where $\hat{\gamma}=(\gamma, g), \hat{\psi}=(\psi, e), \hat{\beta}=(\beta, b)$ and $\hat{\zeta}=(\zeta, f)$ define the rotation angle and sliding distance at the fixed and moving joints, respectively.

The result is that the coordinate transformation $[T]$ that locates the final moving frame $M$ in $F$ is defined in two ways, that is ,

$$
[T]=\left[D_{\text {in }}\right]=\left[D_{\text {out }}\right]
$$

Substitute Eqs. (5.1) and (5.2) into this equation to obtain the kinematics equation of the RCCC chain.

From Eq. (5.3) we can have 9 equations,

$$
\left[\begin{array}{cccc}
f_{1} & f_{2} & f_{3} & f_{7} \\
0 & f_{4} & f_{5} & f_{8} \\
0 & 0 & f_{6} & f_{9} \\
0 & 0 & 0 & 0
\end{array}\right]=\left[D_{\text {in }}\right]-\left[D_{\text {out }}\right]=\left[\begin{array}{cccc}
0 & 0 & 0 & 0 \\
0 & 0 & 0 & 0 \\
0 & 0 & 0 & 0 \\
0 & 0 & 0 & 0
\end{array}\right] .
$$

As discussed in Sec. 4.4.1, the joint variables $\{\phi, \psi, \zeta\}$ will be divided into six separate variables as $\{\cos \phi, \sin \phi, \cos \psi, \sin \psi, \cos \zeta, \sin \zeta\}$. For a given design, the solution of this system of Eqs. (5.4) with nine variables is the motion curve of the linkage, and "real" solutions are those for which satisfy $\cos ^{2} \phi+\sin ^{2} \phi=\cos ^{2} \psi+\sin ^{2} \psi=\cos ^{2} \zeta+\sin ^{2} \zeta=1$.

As with the planar four-bar linkage, the RCCC linkage has two GIs for an arbitrary input. As shown in Fig. 5.2 and Fig. 5.3, A is the revolute joint, crank AG fixed in the ground, and crank AB is the input crank. The input angle $\theta=\pi / 2$, and RCCC linkage shows two different GIs.

\subsubsection{Singularity Points}

From Eqs. (5.4), the singularity condition of RCCC linkage is

$$
f_{10}=\cos ^{2} \theta+\sin ^{2} \theta-1=0
$$




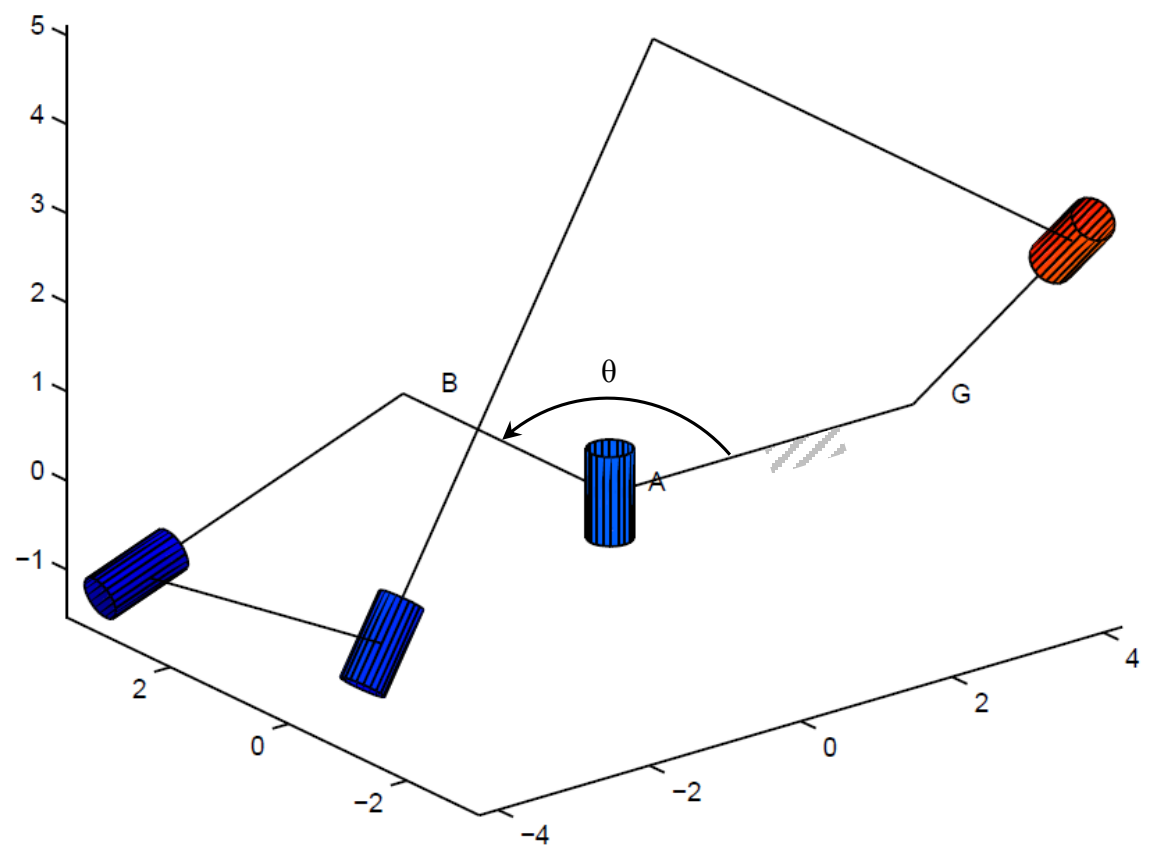

Figure 5.2: RCCC linkage position, GI 1.

and

$$
D:=\operatorname{det}\left[\begin{array}{llllllllll}
\frac{\partial f_{1}}{\partial \cos \phi} & \frac{\partial f_{1}}{\partial \sin \phi} & \frac{\partial f_{1}}{\partial \cos \psi} & \frac{\partial f_{1}}{\partial \sin \psi} & \frac{\partial f_{1}}{\partial \cos \zeta} & \frac{\partial f_{1}}{\partial \sin \zeta} & \frac{\partial f_{1}}{\partial c} & \frac{\partial f_{1}}{\partial e} & \frac{\partial f_{1}}{\partial f} \\
\frac{\partial f_{2}}{\partial \cos \phi} & \frac{\partial f_{2}}{\partial \sin \phi} & \frac{\partial f_{2}}{\partial \cos \psi} & \frac{\partial f_{2}}{\partial \sin \psi} & \frac{\partial f_{2}}{\partial \cos \zeta} & \frac{\partial f_{2}}{\partial \sin \zeta} & \frac{\partial f_{2}}{\partial c} & \frac{\partial f_{2}}{\partial e} & \frac{\partial f_{2}}{\partial f} \\
\frac{\partial f_{3}}{\partial \cos \phi} & \frac{\partial f_{3}}{\partial \sin \phi} & \frac{\partial f_{3}}{\partial \cos \psi} & \frac{\partial f_{3}}{\partial \sin \psi} & \frac{\partial f_{3}}{\partial \cos \zeta} & \frac{\partial f_{3}}{\partial \sin \zeta} & \frac{\partial f_{3}}{\partial c} & \frac{\partial f_{3}}{\partial e} & \frac{\partial f_{3}}{\partial f} \\
\frac{\partial f_{4}}{\partial \cos \phi} & \frac{\partial f_{4}}{\partial \sin \phi} & \frac{\partial f_{4}}{\partial \cos \psi} & \frac{\partial f_{4}}{\partial \sin \psi} & \frac{\partial f_{4}}{\partial \cos \zeta} & \frac{\partial f_{4}}{\partial \sin \zeta} & \frac{\partial f_{4}}{\partial c} & \frac{\partial f_{4}}{\partial e} & \frac{\partial f_{4}}{\partial f} \\
\frac{\partial f_{5}}{\partial \cos \phi} & \frac{\partial f_{5}}{\partial \sin \phi} & \frac{\partial f_{5}}{\partial \cos \psi} & \frac{\partial f_{5}}{\partial \sin \psi} & \frac{\partial f_{5}}{\partial \cos \zeta} & \frac{\partial f_{5}}{\partial \sin \zeta} & \frac{\partial f_{5}}{\partial c} & \frac{\partial f_{5}}{\partial e} & \frac{\partial f_{5}}{\partial f_{5}} \\
\frac{\partial f_{6}}{\partial \cos \phi} & \frac{\partial f_{6}}{\partial \sin \phi} & \frac{\partial f_{6}}{\partial \cos \psi} & \frac{\partial f_{6}}{\partial \sin \psi} & \frac{\partial f_{6}}{\partial \cos \zeta} & \frac{\partial f_{6}}{\partial \sin \zeta} & \frac{\partial f_{6}}{\partial c} & \frac{\partial f_{6}}{\partial e} & \frac{\partial f_{6}}{\partial f_{3}} \\
\frac{\partial f_{7}}{\partial \cos \phi} & \frac{\partial f_{7}}{\partial \sin \phi} & \frac{\partial f_{7}}{\partial \cos \psi} & \frac{\partial f_{7}}{\partial \sin \psi} & \frac{\partial f_{7}}{\partial \cos \zeta} & \frac{\partial f_{7}}{\partial \sin \zeta} & \frac{\partial f_{7}}{\partial c} & \frac{\partial f_{7}}{\partial e} & \frac{\partial f_{7}}{\partial f} \\
\frac{\partial f_{8}}{\partial \cos \phi} & \frac{\partial f_{8}}{\partial \sin \phi} & \frac{\partial f_{8}}{\partial \cos \psi} & \frac{\partial f_{8}}{\partial \sin \psi} & \frac{\partial f_{8}}{\partial \cos \zeta} & \frac{\partial f_{8}}{\partial \sin \zeta} & \frac{\partial f_{8}}{\partial c} & \frac{\partial f_{8}}{\partial e} & \frac{\partial f_{8}}{\partial f} \\
\frac{\partial f_{9}}{\partial \cos \phi} & \frac{\partial f_{9}}{\partial \sin \phi} & \frac{\partial f_{9}}{\partial \cos \psi} & \frac{\partial f_{9}}{\partial \sin \psi} & \frac{\partial f_{9}}{\partial \cos \zeta} & \frac{\partial f_{9}}{\partial \sin \zeta} & \frac{\partial f_{9}}{\partial c} & \frac{\partial f_{9}}{\partial e} & \frac{\partial f_{9}}{\partial f}
\end{array}\right]
$$

It is noted that Eq. (5.5) can be replaced by

$$
f_{11}=\cos ^{2} \phi+\sin ^{2} \phi-1=0,
$$




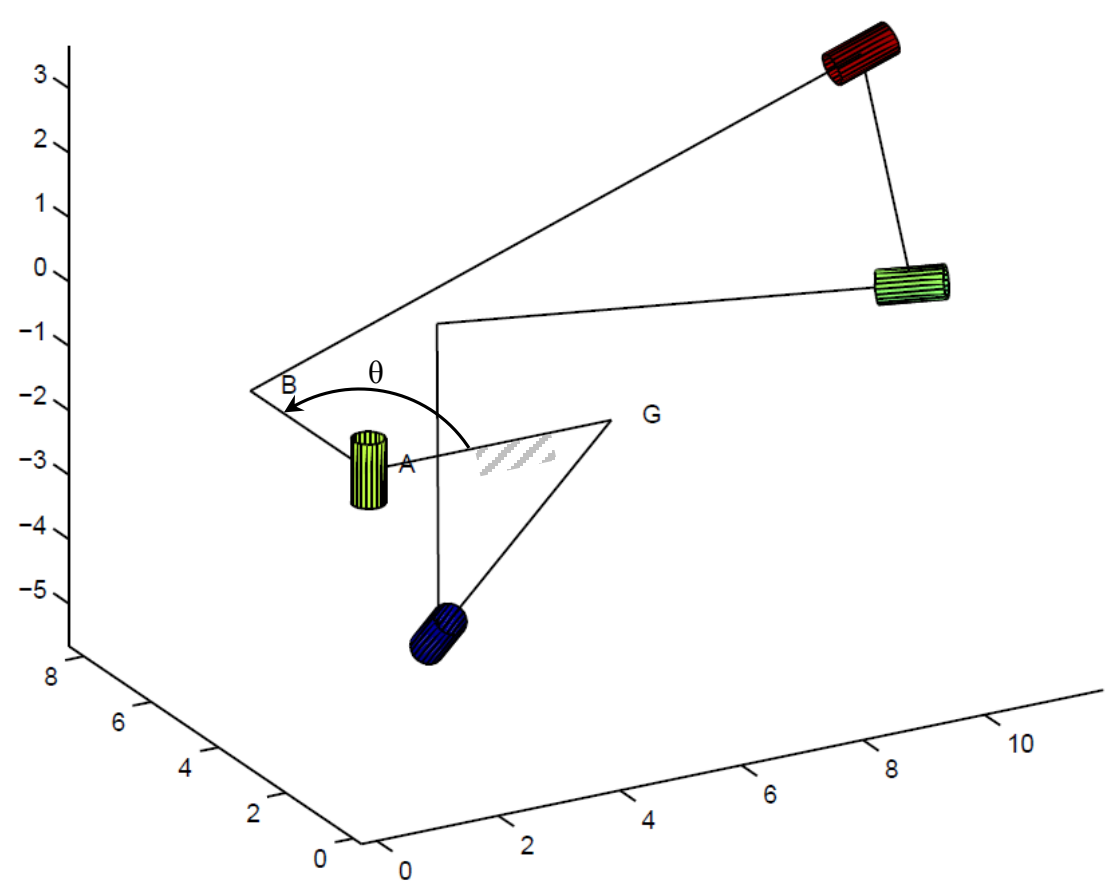

Figure 5.3: RCCC linkage position, GI 2.

or

$$
f_{12}=\cos ^{2} \psi+\sin ^{2} \psi-1=0
$$

or

$$
f_{13}=\cos ^{2} \zeta+\sin ^{2} \zeta-1=0 .
$$

However, it turns out that the solutions for this formulation of the singularities of the RCCC linkage is providing inconsistent results. That is, if we combine Eqs. (5.4) and (5.6) with any equation from Eqs. (5.5), (5.7), (5.8) and (5.9), different sets of solutions are obtained by solving different equations combinations. Additionally, the number of discrete sets of solutions are odd, which is inconsistent with the property that a four-bar linkage has an even numbers of singularities. 
The accuracy of the equation development for RCCC singularity should be verified in the future work.

Another challenge is that the determinant of $D$-matrix produces a very large polynomial function. Using the process discussed in this thesis to construct the critical condition for the RCCC linkage, the $E$-matrix will be $11 \times 11$. The elements in $E$-matrix, which are the derivatives of determinant of $D$-matrix, make the determinant of $E$-matrix extremely large. At present, the research team does not have access to sufficient computational power to complete the calculation of critical points of RCCC linkage under this condition. In the future work, we can try to reduce the mathematical model of this system. Computational resources can also be accessed to the calculations. Another approach is trying to construct the mathematics model in different methods which may not need to divide $\{\phi, \psi, \zeta\}$ into $\{\cos \phi, \sin \phi, \cos \psi, \sin \psi, \cos \zeta, \sin \zeta\}$, this may relieve the calculation process. 


\section{BIBLIOGRAPHY}

[1] Myszka D. Murray A. and Wampler C., 2012, Mechanism Branches, Turning Curves, and Critical Points, Proceedings of IDETC/CIE 2012, DETC2012-70277.

[2] Bates, D.J., Hauenstein, J.D., Sommese, A.J., and Wampler, C.W., Bertini: Software for Numerical Algebraic Geometry, available at http://www.nd.edu/ sommese/bertini.

[3] Erdman, A. Sandor, G. and Kota, S., 2001, Mechanism Design: Analysis and Synthesis, Vol. 1, 4/e, Prentice Hall.

[4] Wampler, C.W., and Sommese, A.J., 2011, Numerical Algebraic Geometry and Algebraic Kinematics, Acta Numerica, 469-567.

[5] Chase, T. and Mirth, J., 1993, Circuits and Branches of Single-Degree-of-Freedom Planar Linkages, ASME Journal of Mechanical Design, vol. 115, No. 2, pp. 223-230.

[6] Foster, D. and Cipra, R., 1998, Assembly Configurations and Branches of Planar Single-Input Dyadic Mechanisms, ASME Journal of Mechanical Design, vol. 120, No. 3, pp. 381-386.

[7] Foster, D. and Cipra, R., 2002, An Automatic Method for Finding the Assembly Configurations of Planar Non-Single-Input Dyadic Mechanisms, ASME Journal of Mechanical Design, vol. 124, No. 3, pp. 58-67.

[8] Mirth, J. and Chase, T., 1993, Circuit Analysis of Watt Chain Six-Bar Mechanisms, ASME Journal of Mechanical Design, vol. 115, No. 2, pp. 214-222.

[9] Davis, H. Chase, T. and Chase, T., 1994, Circuit Analysis of Stephenson Chain Six-Bar Mechanisms, Proceedings of the ASME Design Technical Conferences, DE-Vol. 70, pp. 349-358.

[10] Wantanabe, K. and Katoh, H., 2004, Identification of Motion Domains of Planar Six-Link Mechanisms of the Stephenson-Type, Mechanism and Machine Theory, vol. 39, pp. 10811099.

[11] Ting, K. L., Dou, X., 1996, Classification and Branch Identification of Stephenson Six-bar Chains, Mechanism and Machine Theory, vol. 31, no. 3, pp. 283-295. 
[12] Litvin, F. and Tan, J., 1989, Singularities in Motion and Displacement Functions of Constrained Mechanical Systems, International Journal of Robot Research, vol. 8, No. 2, pp. $30-43$.

[13] Gosselin, C. and Angeles, J., 1990, Singularity Analysis of Closed-Loop Kinematic Chains, IEEE Transactions on Robotics and Automation, vol. 6, No. 3, pp. 281-290.

[14] Murray, A., Turner, M., Martin, D., 2008, Synthesizing Single DOF Linkages Via Transition Linkage Identification ASME Journal of Mechanical Design, Vol. 130, No. 2, 022301.

[15] Myszka, D., Murray, A., Schmiedeler, J., 2010, Using a Singularity Locus to Exhibit the Number of Geometric Inversions, Transitions and Circuits of a Linkage, Proceedings of the ASME International Design Technical Conferences, pp. 771-781.

[16] Innocenti, C. and Parenti-Castelli, V., 1998, Singularity-Free Evolution From One Configuration to Another in Serial and Fully Parallel Manipulators, ASME J. Mechanical Design, Vol. 120, NO. 1, pp. 281-290.

[17] McAree, P.R., Daniel, R.W., 1999, An Explanation of Never-Special Assembly Changing Motions for 3-3 Parallel Manipulators, The International Journal of Robotics Research, Vol. 18 , NO. 6, pp. 556-574.

[18] Wenger, P., Chablat, D., Zein, M., 1998, Workspace and Assembly-modes in Fully Parallel Manipulators: A Descriptive Study, Advances in Robot Kinematics, Kluwer Academic Publishers, pp. 117-126.

[19] Wenger, P., Chablat, D., Zein, M., 2007, Degeneracy Study of the Forward Kinematics of Planar 3-RPR Parallel Manipulators ASME Journal of Mechanical Design, Vol. 129, No. 12, pp. 1265-1268.

[20] Zein, M., Wenger, P., Chablat, D., 2006, Singular Curves and Cusp Points in the Joints Space of 3-RPR Parallel Manipulators, Proc of IEEE International Conference on Robotics and Automation.

[21] Zein, M., Wenger, P., Chablat, D., 2006, Non-singular Assembly Mode Changing Motions for 3RPR Parallel Manipulators, Advances in Robot Kinematics, Vol. 43, NO. 4, pp. 480-490.

[22] Macho, E., Altuzarra, O., Pinto, C.,Hernandez, A., 2008, Transitions between Multiple Solutions of the Direct Kinematics problems, Advances in Robot Kinematics, Springer Netherlands, Part 5, pp. 301-310.

[23] McCarthy, J. M. and Soh, G.S., 2011, Geometric Design of Linkages, 2/e, Springer.

[24] Wampler, C.W., 1996, Isotropic Coordinates, Circularity, and Bezout Numbers: Planar Kinematics from a New Perspective, Proceedings of the ASME Design Technical Conference, Paper 96-DETC/MECH-1210. 
[25] Park, F.C., Kim, J.W., 1999, Singularity Analysis of Closed Kinematic Chains, ASME J. Mechanical Design, Vol. 121, pp. 32-38.

[26] Myszka, D., Murray, A., Schmiedeler, J., Singularity Analysis of an Extensible Kinematic Architecture: Assur Class N, Order N-1, Journal of Mechanisms and Robotics, ASME, 2009, Vol 1, No.1, 011009.

[27] Wampler, C.W., 1999, Solving the Kinematics of Planar Mechanisms ASME Journal of Mechanical Design, Vol. 121, 387-391.

[28] Wampler, C.W., 2001, Solving the Kinematics of Planar Mechanisms by Dixon Determinant and a Complex-Plane Formulation, ASME J. Mechanical Design, Vol. 123, 3382-387.

[29] Nielsen, J., and Roth, B., 1999, Solving the Input/ Output Problem for Planar Mechanisms, ASME J. Mech. Design, Vol. 121, No. 2, 206211.

[30] Sommese, A.J., and Wampler, C.W., 2005, Numerical Solution of Systems of Polynomials Arising In Engineering And Science, World Scientific Press, Singapore.

[31] Lu, Y., Bates, D.J., Sommese, A.J., and Wampler, C.W., 2007, Finding all Real Points of a Complex Curve, In Proc. Midwest Algebra, Geometry and Its Interactions Conf., Contemporary Mathematics, AMS, Vol. 448, 183-205.

[32] Besana, G.M., Di Rocco, S., Hauenstein, J.D., Sommese, A.J., and Wampler, C.W., 2011, Cell decomposition of almost smooth real algebraic surfaces, preprint.

[33] Allgower, E.L., and Georg, K., 1997, Numerical Path Following, In Handbook of numerical analysis, Vol. V, 3-207, North-Holland, Amsterdam. 